DE86 009612

\title{
Spectroscopy of GaAs Quantum Wells
}

\author{
Lawrence Camest West
}

\author{
Manuscript date: July 1985
}

\author{
DISCLAIMER
}

This report was prepared as an account of work sponsored by an agency of the United States Government. Neither the United States Government nor any agency thereof, nor any of their employees, makes any warranty, express or implied, or assumes any legal liability or responsibility for the accuracy, completeness, or usefulness of any information, apparatus, product, or process disclosed, or represents that its use would not infringe privately owned rights. Reference herein to any specific commercial product, process, or service by trade name, trademark, manufacturer, or otherwise does not necessarily constitute or imply its endorsement, recommendation, or favoring by the United States Government or any agency thereor. The views and opinions of authors expressed herein do not necessarily state or reflect those of the United States Government or any agency thereof.

\section{LAWRENCE LIVERMORE NATIONAL LABORATORY University of California - Livermore, California - 94550}


Spectroscopy of GaAs Quantum Wells

\author{
A DISSERTATION \\ SUBMITTED TO THE DEPARTMENT OF APPLIED PHYSIC'S \\ AND TIE COMMITTEE OIN GRADUATE STUDIES \\ OF STANFORD UNIVERSITY \\ IN PARTIAL, FULFILLMENT OF THE REQUIREMENTS \\ FOR THE DEGIREE OF \\ DOCTOR OF PHLOSOPHY
}

\author{
by \\ Lawrence Camest West \\ July 1885
}


(C) Copyright 1985

by

Lawrence Camest West

The Goverment reserves for itself and others acting on its behalf a royalty free, nonexclusive, irrevocable, world-wide license for governmental purposes to publish, distribute, translate, duplicate, exhibit, and perform any such data ooprighted by the contractor. 
I certify that 1 have read this thesis and that in my opinion it is fully adequate, in scope and quality, as a dissertation for the degree of Doctor of Philosophy.

Prof. R. L. Byer

Principal Advisor

Dept. of Applied Physics

I certify that I have read this thesis and that in my opinion it is fully adequate, in scope and quality, as a dissertation for the degree of Doctor of Philosophy.

Prof. W. A. Harrison

Dept. of Applied Physics

I certify that I have read this thesis and that in my opinion it is fully adequate, in scope and quality, as a dissertation for the degree of Doctor of Philosophy.

Prof. J. S. Harris

Dept. of Electrical Engineering

Approved for the University Committee on Graduate Studies:

Dean of Graduate Studies and Research 


\begin{abstract}
A new type of optical dipole transition in GaAs quantum wells has been observed. The dipole occurs between two envelope states of the conduction band electron wavefunction, and is called a quantum well envelope state transition (QWLST). The QWEST is observed by infrared absorption in three different samples with quantum well thicknesses 65,82 , and $92 \AA$ and resonant energies of 152,121 , and $108 \mathrm{meV}$, respectively. The oscillator streingth is found to have values of over 12 , in good agreement with prediction. The linewidths are seen as narrow as $10 \mathrm{meV}$ at room temperature and $7 \mathrm{meV}$ at low temperature, thus proving a narrow line resonance can indeed occur between transitions of free electrons. Techniques for the proper growth of these quantum well samples to enable observation of the QWEST have also been found using (AlGa)As compounds.

The quantum dipole matrix element of the QWEST is found to have a size of nearly $20 \mathrm{e} \AA$. This extremely large dipole, in combination with the narrow resonance linewidth, give the QWEST very strong nonlinear properties. Because both the lower and upper quantum well states consist of free electrons in the same conduction band, an electron can relax from the upper to the lower energy state by an elastic scattering, requiring no energy transfer. This property enables the transition to have an extremely fast transition time, predicted to be subpicosecond.

This QWEST is considered to be an ideal material for an all optical digital computer. The QWEST can be made frequency matched to the inexpensive Carbon Dioxide laser with an infrared wavelength of 10 microns. The nonlinearity and fast relaxation time of the QWEST indicate a logic element with a subpicosecond switch time can be built in the near future, with a power level which will eventually be limited only by the noise from a lack of quanta to above approximately 10 microwatts.
\end{abstract}




\section{Acknowledgements}

The credit for getting me into trouble undoubtedly goes to Dr. Lowell Wood of LLNL. He first suggested to me the possible advantages of optics for high spend supercomputing. He also succeeded in convincing me, when I was too young to know better, that not only was an optical digital computer possible, but I'd be able to turn in a working model for my Ph.D. thesis. (As my graduation date began to extend excessively, this became a fear rather than a hope.) On several occasions, when I came to my sens's, I would approach Lowell to inform him that the concept of an optical computer was fundamentally flawed and I was going to work on a reasonable thesis with a Stanford Professor. Lowell would understand, but demand an explanation as to the fundamental difficulties. He would then carefully pick through and destroy all my assumptions and then cleverly suggest I simply clean up my reasons before turning to other problems. Of course, in this cleaning up these assumptions I would make several discoveries and continue working on the problem. His role as advocate was a combination of support and scientific stimulation. This support was given without regard to personal benefit. He was interested in seeing the technological stature of the world improve dramatically and felt I was a means to this end. He merely had to convince me of my role. I now understand that in many ways Lowell was right. In fact, in some aspects, the results have exceeded both our expectations. For instance, optical logic is now' seen to be not only capable of being faster than electronic logic, but lower power as well. On the other hand, the Ph.D. thesis will not be a working optical supercomputer. I do not even have a working logic element yet. Instead, I only have the material which will allow an optical computer to happen along with the key tricks for realizing optical logic elements and their interconnects in a real system. However, had Lowell not consistently had faith in me and a solution, and prodded me to try what a sane person would consider 
crazy, I very likely would have done a good "reasonable" thesis. But I would not have attempted the "impossible." I inus sincerely wist to thank Lowell for this continued and very strong intellectual, emotional, and financial support, without which I would not have attempted to do nearly as much as I found could indeed be accomplished.

The credit for getting me out of trouble clearly goes to Prof. Robert Byer at Stanford University. He knew from an early date enough about me to know I was going to be a problem, especially considering my past interaction with the department of Applied Physics. But he nevertheless made a personal choice to help me, even though I was clearly not going to be of direct aid to his career, and more likely a burden. But Dr. Byer's assistance was not merely one of ideological support. I am especially grateful that Dr. Byer not only allowed me the freedom to continue my quest for optical logic, but provided much needed guidance. This guidance was multifold. Dr. Byer would provide scientific advice as to possible directions towards creation of any of my latest designs. He provided me with guidance on how to concuct a proper scientific investigation, including specific advice on approaclsus to a desired measurement. He taught me how to write proper scientific papers and present my results in a manner acceptable to the scientific community. And rnost importantly, he pinned me down to a thesis topic. But, through all, he had enough faith in me to allow me near total freedom to pursue optical logic and develop ideas while guiding me to the Ph.D.. Dr. Byer spent an appreciable amount of time directing me and guiding me towards a better path with little regards to the effect on his personal career. For this I wish to express my deep gratitude to this man.

A good part of the credit for the realization of the QWEST in GaAs quantum wells goes to Steve Eglash. He was willing to take time off a Stanford $\mathrm{Ph} . \mathrm{D}$ pursuit of his own to develop proper growth procedures for these quantum well samples. He 
spent a great deal of time on the growth of these samples and their characterization, in addition to discussing methods which finally lead to the successful observation of the QWEST.

I wish to thank Peter Hagelstein and Rod Hyde of LLNL for stimulating discussions during which much new understanding was found. They also provided much encouragement when both physical progress and problems at Stanford seemed insolvable.

I wish to thank Prol. Goodman, who was my temporary advisor when I needed time to develop new ideas for optical logic, which lead to the QWEST.

I wish to thank Dave Tuckerman at LLNL for assistance in photolithograply of these quantum well samples.

I wish to thank Curly Vanderbeck at LLNL who used his advanced mechanical knowledge and skill to construct much of the apparatus used for these experiments.

I wish to thank Profs. W. Harrison and J. Harris for reading this manuscript.

I wish to thank C. Wriston for suppori and understanding.

Two institutions also were important in allowing this work to continue. The first is Lawrence Livermore National Laboratories, which provided me with a salary and a laboratory in which to perform this research. The other is Hewlett-Packard, which allowed these quantum well samples to be grown with their facilities. 
l.Introduction 1

I.Background

A. Gallium Arsenide Quantum Wells 5

B. Quantum Well Energies 8

C. Quantum Well Density of States 10

D. Two-Dimensional Excitons 13

E. Interband Spectra $\quad 17$

F. Inelastic Light Scaltering $\quad 20$

G. Intersubband Transitions in Other 2-D Systems 25

III.Infinite Barrier and Parabolic Energy Bands

A. The Envelope State Transition 27

B. The QWEST Dipole $\quad 28$

C. The QWEST Energy 32

D. The QWEST Oscillator Strength 33

IV.Quantum Wells in (AIGa)As Crystals

A. Band Structure in GaAs $\quad 37$

B. GaAs Quantum Well Energy 43

C. GaAs QWEST Dipole and Oscillator Strength 57

D. Energy Broadening Mechanisms $\quad 70$

E. Energy Shift Mechanisms $\quad 86$

F. Optical Properties $\quad \boldsymbol{9 . 1}$

V.QWEST Absorption Experiments

A. Mechanics of the Optical Probe 09

B. (AlGa)As QW Crystal Development 111

C. Optical Observations of the QWEST 140

$\begin{array}{ll}\text { D. Analysis of Observations } & 150\end{array}$

IX. Conclusion and Future Applications $\quad 159$

$\begin{array}{ll}\text { References } & 162\end{array}$

Appendices

Appendix A: Evaluation of Coulomb Potential Integrals $\quad 169$

Appendix B: Design of an Optical Digital Computer 172 


\section{List of Figures}

Figure II.1: A plot of the density of states vs. electron energy for a quantum well.

Figure II.2: Absorption spectra of a GaAs quantum well at $77 \mathrm{~K}$ both before and 100 picoseconds after pumping to an electron density of $5 \times 10^{11} \mathrm{~cm}^{-2}$. Note that the excitons disappear with the presence of the free electrons because of the shiclding of the coulomb potential (from Ref.[27]).

(around eq. (28)).

Figure II.3: A plot of the conduction and valence bands of GaAs, including a possible inelastic scattering.

Figure III.1: The potential and wavefunction of an infinite depth well.

Figure III.2: Density of QWEST states for an infinite well.

Figure IV.1: A diagram of an actual quantum well in (AlGa)As materials. The potentials and two lowest envelope wavefunctions are illustrated.

Figure IV.2: A plot of the calculated QWEST energies using the $\mathrm{m}^{*}$ and normal boundary conditions with the experimental observations also indicated.

Figure IV.3: a.) A plot of the quantum well energies versus transverse $k$ vector for the two lowest energy quantum well states. b.) A plot of the the magnitude of QWEST energy reduction from the $k_{t}=0$ value as a function of $k_{t}$.

Figure IV.4: a.) A plot of QWEST density of states for $65 \mathrm{~A}$ well at $35 \mathrm{~K}$. b.) QWEST density of states for a $65 \mathrm{~A}$ well at $300 \mathrm{~K}$. c.) QWEST density of states for an $82 \mathrm{~A}$ well at $300 \mathrm{~K}$.

Figure IV.5: A diagram of the electron path during its relaxation from the upper quantum well state. The initial process is an elastic scattering to the lower band, followed by a slower inelastic decay to the lattice temperature. The upper plot is of the allowed wavevectors of a quantum well. The transverse wavevectors are sufficiently close to be drawn as a line. Note the lowest energy quantum well is the $\mathrm{n}=1$ state. A constant energy surface is also in dicated. The lower plot illustrates the energy of the two lowest quantum well states as a function of transverse wavevector.

Figure IV.6: A plot of the 2-D exciton linewidth of various quantum well samples as a function of substrate growth temperature (from Ref. [31]).

Figure V.1: Nicolet spectrometer configuration. 
Figure V.2: Sketch (a) shows the polarization and angle of the $3 \mathrm{~mm}$ diameter infrared beam with respect to the sample. Note the internal electric field has only a small component perpendicular to the surface because of the strong refraction. Sketch (b) shows the elliptical beam pattern on the surface when the sample is at Brewster's angle $\left(\theta=73^{\circ}\right)$. The angle between the major axis of the ellipse and an arbitrary axis in the crystal plane is shown as $\phi$.

Figure V.3: An example of a typical infrared absorption spectra of a GaAs substrate with epitaxial growth of (AIGa)As quantum wells. This sample, A-516, failed to show any QWEST resonances, but exhibites the two small phonon abosrption peaks at 708 and $770 \mathrm{~cm}^{-1}$ seen on all of the later samples. Note that (AlGa)As materials have very little absorption in a broad region of the infrared from 2 to 20 micron wavelengths. (Absorbance $=-\log _{10}($ tranemission $)$ )

Figure V.4: Layer structure of the epitaxial quantum well growth for the earlier samples.

Figure V.5: Infrared absorption spectra of quantum well samples at $\theta=73^{\circ}$ (except where indicated) and at room temperature. a.) Sample A-494, b.) Sample A-495, c.) Difference spectra of samples A-495 and A-494, d.) Sample A-495, two days after spectra b.) was taken (note the differences), e.) Sample A-495 at normal incidence $(\theta=0), f$.) Sample A-495 two months after spectra b.) (note the energy shift of one peak).

Figure V.6: A very high resolution TEM photograph of sample A-495 in the [011] direction, showing atomic resolution of the well and barrier regions and indicating a high quality interface has been created. The $\mathbf{1 0} \mathrm{nm}$ marker 's slightly incorrect, the thickness of the black well region is actually about 33 half cells of the FCC lattice, or 93.277 $\AA$. (Photograph courtesy of F. Ponce at Hewlett-Packard Laboratories)

Figure V.7: A lower magnification of Fig. V.6 showing several of the 50 quantum wells grown for sample A-495.

Figure V.8: A plot of the photoluminescence intensity of various quantum well samples as a function of the substrate growth temperature. Annealing of the samples, at temperatures up to $750 \mathrm{C}$ for up to 4 hours, was found to increase the photoluminescence intensity as shown (from Ref. [31]).

Figure V.0: Layer structure of the epitaxial quantum well growth for the later samples. 
Figure V.10: Photoresist mask of Van der Pauw and Hall probe measurements to determine the electron density in the quantum well samples. The overall width of the entire pattern as etched was about $11 \mathrm{~mm}$. Thus each probe pattern was slightly over $1 \mathrm{~mm}$.

Figure V.11: An SEM photograph of the metalization overlay of a pad of a Hall probe etch in sample B-133. Note that the 7 micron deep etch clearly exposed the quantum well layers, seen as a darker coating about 3 microns deep, at an angle so as to provide some area of contact to the metalization for each of the 50 wells independently. Also note that some of the metalization over the pads extended down the edge of the etched region from improper liftoff of this metal. A larger view shows the etched regions shows that the metal extension cuts off completely at several spots. Also, since the upper surface is insulating, the only effect of this extra metal would be to short contact pad to one another. This was not observed.

Figure V.12: Infrared absorption spectrum of sample B-331 and B-3.32 at $\theta=$ $73^{\circ}$ and at room temperture. $\mathrm{mAbs}=-10^{-3} \log _{10}$ (Transmission). Spectrum (a) is of sample B-332, a $65 \AA$ quantum well, and was independent of rolation about $\phi$. Spectra (b) and (c) are both of sample B-331, a $82 \AA$ well on average. with $\phi=0^{\circ}$ (b) and $\phi=90^{\circ}$ (c), showing sample inhomogeneity with scan direction (see Fig. V.2).

Figure V.13: Absorption spectrum of sample B-332, a $65 \AA$ quantum well, at room temperature with (a), $\theta=73^{\circ}$, and (b), $\theta=0^{\circ}$. Note the QWEST absorption peak disappears when the elertric feld vector of the infrared beam is rotated to lie completely in the sample plane, as expected. The shallow baseline slope is an artifact of the fourier transform process of the spectrometer in the presence of small jitter in the zero point of the interferogram, and should be ignored as noise.

Figure V.14: Infrared absorption spectra of B-331-B at room temperature and $\theta=73^{\circ}$ as function of rotation angle, $\phi$, with $\phi$ indicated for each of the spectra.

Figure V.15: Infrared absorption spectra of sample B-332-A at $\theta=73^{\circ}$ and several temperatures.

Figure V.16: Infrared absorption spectra of B-332-A at $\theta=73^{\circ}$ for 34 and 294 K.

Figure V.17: Infrared absorption spectra of B-332-A at $\theta=73^{\circ}$ for 280 and $41 \mathrm{~K}$. This spectra is not redrawn so as to retain the original noise of the GaAs substrate and epitaxial layers. Note that except for the QWEST, the spectrum is 
devoid of absorption peaks at this level. The noise present is from two sources. The first is residual $\mathrm{CO}_{2}$ in the spectrometer, which is the source of the broad bumps at $3000 \mathrm{~cm}^{-1}$ for the $41 \mathrm{~K}$ plot and the numerous peaks around $1600 \mathrm{~cm}-1$. The $\mathrm{CO}_{2}$ spectrum actually consists of numerous $1 \mathrm{~cm}^{-1}$ wide peaks, but the spectra here are taken with too low a resolution to see the individual peaks. In addition, high frequency oscillations, from the cavity resonance formed by reflections of the outer wafer surfaces which are not quite removed at Brewster's angle, are seen at the higher wevienumbers.

Figure V.18: The infrared absorption spectrum of sample B-331-B at $\theta=73^{\circ}$ and $\phi=0^{\circ}$ on two different dates.

Figure V.19: The infrared absorption spectrum of sample B-332-A at $\theta=73^{\circ}$ and $\phi=0^{\circ}$ on two different dates.

Figure B.1: The desired transfer functions of a logical identity and inverter operation.

Figure B.2 An example of a transfer function of an optical cavity containing a nonlinear material.

Figure B.3: An example of a complementary NOR optical logic gate, also illustrating several interconnection elements such as right angle corners and crossthroughs.

Figure B.4: An example of a scattering-coupled interferometer.

Figure B.5: A possible optical computer physical layout. 


\section{List of Tables}

Table IV.1: Broadening effects for a $65 \AA$ quantum well at $300 \mathrm{~K}$.

Table IV.2: Broadening effects for a $65 \AA$ quantum well at $35 \mathrm{~K}$.

Table IV.3: The QWEST energy and energy shifts for quantum wells with thicknesses of 65,82 and $82 \AA$. Energies are in units of meV.

Table V.1: Comparison of observed QWEST energies with theoretical expectation. Energies are measured in $\mathrm{meV}$.

Table V.2: A possible interpretation of the broadening effects for a 65 . quantum well at $300 \mathrm{~K}$.

Trble V.3: A possible interpretation of the broadening effects for a $65 \hat{\Lambda}$ quantum well at $35 \mathrm{~K}$. 


\section{Introduction}

With the advent of quantum mechanics, many classical phenomena were found to give better agreement with experiment when reinterpeted in the quantum mechanical formalism. In addition, many new phenomena were discovered with no classical analog. Quantum wells fall in the later category. Quantum wells are described by nothing more than the kinetic term of Schrodinger's equation and boundary conditions.

A particle in one dimension trapped between two high potential barriers is said to be in a well. The resultant quantization of states and energy is rarely obscrved directly because dimensions as small as a micron have energies easily washed out by thermal and other effects. However within the last few years it has become possible to grow alternate layers of $\mathrm{GaAs}$ and (GaAl)As with dimensions of 0.5 nanometers. With layers of this dimension, the GaAs acts as the well and the (GaAl)As acts as the barrier to either the electron or hole. With these small layers and small mass of the electron in $\mathrm{GaAs}$, the energies become a fraction of an electron volt and are observable.

However, these quantum wells are not completely one dimensional. The electrons are still free to move about wishin the layer and this degree of freedom adds an aroitrary energy to the quantum state. In the past these quantum well stales have been optically observed by several mechanisms, all of which involve conductionto-valince band transitions. For instance, single photon atısorption experiments on an insulating GaAs "rantum well at low temperature has revealed a jump in the density of states as we energy is scanned during an optical transition through each quantum state. Additionally, an electron and a hole can form a bound staie in two dimensions called a two dimensional exciton. This state has a narrow bandwidth and a bound state energy of about $10 \mathrm{meV}$. Thus one 2-D 
exciton is seen for each quantum well state at an energy just below the jump in density of states. These effects will be discussed further in the next chapter.

These single photon absorption experiments involve transitions between the conduction band and valence band. Thus the energy of the quantum well state was only observed as an incremental addition to the band energy. However, theory predicts that a direct transition can be observed between quantum well states. This transition occurs between two states which are both in the conduction band and differs significantly from band-to-liand transitions.

A quantized state in a crystal can be described by the multiple of the wavefunctions of two states, the Bloch state and the envelope state. The Bloch state (also called Bloch function) is periodic with the lattice spacing. The envelope state describes the non-periodic behavior. Whereas a band-to-band transition uses a ? dipole between the Bloch states with the envelope states (or momentum) remaining constant, the transition reported here uses a dipole between envelope states with the Bloch states remaining constani. For these reasons, this transition between quantum well states wili be called a Quantum :Vell Envelope State Transition (or QWEST ).

The envelope state transiticn has several significant characteristics of special interest in optical applications. First, the spread in energy of the quantum well siates from the transverse kinetic energy is cancelled for the transition when momentum is conserved and the energy band has a quadratic dependence on the wavevector magnitude. Both of these conditions are valid for experinents performed. As a result, the transition has a very narrow bandwidth. Second, the dipole matrix element is between the envelope states. Since these envelope states are fairly large ( 5 - 10 nanometers), when compared with atomic or Bloch states ( 0.05 to 0.3 nanometers ), the dipole is also very large. This large dipole in turn yields a strong absorption 
and $\chi^{(3)}$ nonlinear effects, which vary as the second and fourth power, respectively, of the dipole matrix element. Third, the relaxation time of the transition is on the picosecond time scale, allowing high speed optical nonlinear interactions.

It is interesting to note the QWEST was not discovered out of a desire to demonstrate quantum physics or an extension of work with quantum wells. Instead, the QWEST discovery arose out of the need for a narrow transition with a large dipole a:d fast relaxation time for optical logic. Because of the requirement for picosecond relaxation, a transition was needed between two states in the same band. It was realized that two quantized states in the same band could have significant energy difference and a large dipole if the crystal were small and the effective mass were small. A year was spent exploring ways to grow spherical crystals 15 nanometers in diameter with high precision and regularity. Although this growth was possible with single element crystals, only binary crystals bad sufficiently small effective masses. Possible techniques for growth and sorting of good quality nanocrystals were found, but would take years to fully develop. These difficulties motivated a closer investigation of the quantum well substrates, which had been grown for ten years and were fully developed, giving good quality and high precision. The broadening of the energies from the lack of confinement in the transverse dimension was originally thought to lead to poor absorption and poor nonlinearities from the resultant transition broadening. Upon realizing the transition was not

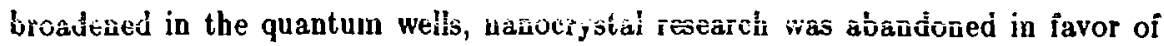
the QWEST.

This development of the QWEST is only a part of a long and continuing attempt to enhance the processing capability of todays electronic computers by many orders of magnitude by use of optics instead of electronics. This research began with the construction of supersonic jet streams to carry away the extreme heat generated 
by the high optical powers needed. The nanocrystals were originaliy investigated because their reduced Rayleigh scattering allowed use of semiconductors in a jet stream. The advances, some of which are seen in this manuscript, caused by investigation of the properties of matter at small sizes has eliminated the need for jet streams for cooling. In fact, it now appears as though this understanding will now lead to optical switching which is limited in power only by the need for sufficient quanta to define a signal. (See Appendix B. for details.)

The QWEST is seen to be breakthrough for the purpose of optical computing. The advantages for optical logic are both physical and technical. The physical advantages of the QWEST l:? in the low power nonlinear properties, provided by its large dipole and its narrow bandwidth, and its very fast relaxation time. The engineering advantages of the QWEST are multifold. The QWEST energies are in the infrrred where inexpensive laser sources can be found and the GaAs materials in whic it is constructed are sufficiently transparant to allow creation of waveguides in the same material. The QWEST energy is tunable to allow use of both signs of nonlinearity within the same logic element. Furthermore, a fully developed semiconductor technology has evolved which can be converted to optical logic fabrication with very little alteration. Logic designs based on the QWEST eppear capable of a thousand-fold improvement over todays elrctronic logic in both speed and power simultaneously, or subpicosecond switch times at microwatt power levels, all at room temperature operation. An overview of an optical digital computer based on the QWEST is given in Appendix B. 


\section{II.Background}

\section{A. Gallium Arsenide Quantum Wells}

The discussion of an infinite one dimensional quantum well is often used as a simple example in beginning quantum physics. ${ }^{[1.2 \mid}$ This elementary example takes on a particularly important realization with the advent of the ability to grow crystals with one dimension as small as nanometers, giving very significant quantized energy differences. The best quality quantum weils have been grown with alternate layers of $\mathrm{GaAs}$ for the well and $\mathrm{Ga}_{1-\mathrm{x}} \mathrm{Al}_{\mathrm{X}} \mathrm{As}(0 \leq \mathrm{x} \leq 1)$ for the barrier to the electron, $|3,4|$ The confinement of the electron occurs because the bandgap of $\mathrm{Ga}_{1-\mathrm{x}} \mathrm{Al}_{\mathrm{x}} \cdot \mathrm{As}$ is givell by ${ }^{\mid 8]} \mathrm{E}_{g}=1.424+1.247 \mathrm{x}(\mathrm{eV})$ for $\mathrm{x}$ less than 0.45 . Above this concentration of aluminum, the bandgap becomes indirect and the above formula is invalid. The heterojunction in ( $\mathrm{GaAl})$ As compounds sustains $65 \%$ of the bandgap difference as a jump in the conduction band potential, and $35 \%$ as a jump in the valence band potential. ${ }^{[23]}$ Thus the potential barrier height between GaAs and (GaAl)As for the conduction band electrou is given by $\Delta V=0.81 \times(\mathrm{eV})$.

The crystal structure of GaAs consists of two interpenetrating face-centered cubic lattices, one lattice each of $\mathrm{Ga}$ or As respectively, creating a zinc-blende structure. The gallium lattice site can be occupied instead by an aluminum atom, with no change in crystal structure and little crystal expansion, in any concentration up to $100 \%$, or pure AlAs.

A number of significant reasons are responsible for the ability to grow excellent quality quantum wells with (GaAl)ess compounds. First, for a variety of reasons, GaAs has become a very common growth material, especially for microwave transistors and diode lasers. This large commercial interest has led to a rich data base on the properties and processing of (GaAl)As compounds. More importantly, numerous facilities now exist which are devoted to the growth and analysis of 
good quality (GaAl)As materials. During the last decade, many of these facilities have given strong attention to growth of $(\mathrm{CaAl})$ As quantum wells. This attention arises from the quantum well's higher electron mobility, of use in making high speed transitors, $[7]$ and in significantly improving the performance of diode lasers. $4 \mid$ The best quantum well structures have been grown ${ }^{|8|}$ either by Molecular Beam Epitaxial[3] (MPE) or Organometallic Chemical Vapor Deposition(a) (OMCVD) techniques. These epitaxial growth techniques allow the freedom to grow planar matcrials with arbitrary choice of doping or lattice composition in the vortical dimension. An abrupt change in material composition in less distance than one lattice spacing is called an abrupt heterojunction and is required for quantum well manufacture. Typically, two materials at a heterojunction have different lattice sizes (and sometimes different lattice structure) which leads to stress at the interface and dislocations, in general giving poor materials. But fortunately, GaAs and AlAs have very similar lattice spacings ( $5.642 \AA$ and $5.661 \AA$ respectively ), with only a $0.34 \%$ lattice mismatch. This mismatch is even less for experimental conditions to be described, with only $30 \%$ of the gallium atoms substituted by aluminum atoms in the $\mathrm{Al}_{\mathrm{x}} \mathrm{Ga}_{1-\mathrm{x}} \mathrm{As}$ electron barrier regions. A particular advantage to the ternary (GaAl)As compounds is this good lattice size match for the substitution of only one site, whereas more generally this match is obtained only with the use of quaternary compounds. This advantage greatly eases the manufacture of these compounds. The growth of good quantum wells is aided by the isoelectronic nature of the $\mathrm{Al}$ and $\mathrm{Ga}$ atoms which lie in the same column of the periodic iable in adjacent rows. Thus the electronic structure of the two compounds is very similar. In particular, both compounds are insulating, which is fortunate since the high Al doping required to create a significant barrier to the electron would otherwise dominate the electronic properties of the sample. The structures are also very stable, with very 
iow interdiffusion of the $\mathbf{A l}$ and $\mathrm{Ga}$ atoms. Quantum well structures are observed to operate in devices at elevated temperatures for 10's of thousands of hours with little degradation. [82]

The interface, or heterojunction, in these ( $\mathrm{GaA})$ As growths is very smocth, with deviations in well thickness typically less than 0.3 nanometers. $[5,6]$ The smoothness of the surface results from the thermodynamics of epitaxial crystal growth. During the epitaxial growth, the deposited atoms of gallium, aluminum, or arsenic are given a thermal energy sufficiently low to allow a high probability of remaining bound to the surface. But this thermal energy is sufficiently high to permit the atom to move about the surface. When this atom now encounters a lattice site discontinuous with the planar surface, the binding enargy of the atom increases since the atom is now bound on more than one side. If the increased binding energy is sufficient, the atom has strong preference to bind to the irregularities, smoothing the surface. If the growth rate is held sufficiently low, then the growth occurs on a previously established irregularity. Thus after smoothing all initial irregularities, the growth completes one atomic plane ( about $2.8 \AA$ for a GaAs atomic pair in the [100] plane ) before initiating a new atomic plane of growth. The summation of all these prosesses in an optimal situation can lead to growth of an epitaxial layer extending centimeters in each transverse dimension without a single jump to the next atomic lattice plane. In practice, occasionally one os two lattice spacing jumps with if order $300 \AA$ of lateral extent are seen. $|5,0|$ These surfaces contain local imperfections ( of order 1 micron in size) from the radial growth of imperfections on the surface. In a clean growth chamber with a good starting substrate, these imperfections are few in number, with the fractional area of these imperfections usually very much less than $0.01 \%$. 


\section{B. Quantum Well Energies}

The simplest example of a quantum mechanical state is the particle in a box in one dimension. Suppose a particle is trapped in an energy potential described by $V(z)$ where $V(z)$ is given by $V(z)=0$ for $|z| \leq L_{z} / 2$ and $V(z) \rightarrow \infty$ for $|z|>L_{z} / 2$ .The Schrödinger equation is given by ${ }^{[1,2]}$

$$
i \hbar \frac{\partial \psi}{\partial t}=-\frac{\hbar^{2}}{2 m} \nabla^{2} \psi+V(\vec{r}) \psi
$$

where $\psi$ is the particle wavefunction, $m$ is the particle mass, and $\hbar$ is Planck's constant. Since Schrödinger's equation is separable in the coordinates for th is potential, only the $z$ dependance is solved here. The other coordinates are considered in the next section. Solving equation (II.1) for constant energy with the one dimensional potential above, this equation reduces in one dimension to[1,2]

$$
-\frac{\hbar^{2}}{2 m} \frac{d^{2} u(z)}{d z^{2}}=E u(z) \quad|z| \leq L_{z} / 2
$$

where $E$ is the energy of the state and

$$
\psi(z, t) \equiv u(z) e^{-i E t / \hbar}
$$

is the definition of $u(z)$, which has no time dependence. For $|z|>L_{z} / 2$, the potential demands $u(z)=0$. This requirement also gives the boundary conditions $u\left(L_{z} / 2\right)=$ $u\left(-L_{z} / 2\right)=0$. The wavefunctions are then quantized and equation (II.2) has the solution given by $[1,2]$

$$
u_{n}(z)=\sqrt{\frac{2}{L_{z}}} \sin n \frac{\pi}{L_{z}}\left(z+L_{z} / 2\right)
$$

where $\mathrm{n}$, a positive integer, is the quantum number of the state $u_{n}(z)$. 
Similar quantum well $s \div$ ates are often used in solid state theories of electrons in larger crystals but are never observed as a single state. In fact, because the states are never observed individually, the true wavefunctions are rarely used in solid state theory. Instead solid state theorists use a more convenient wavefunction which is continuous in value and slope in wrapping around frem opposite boundries. This commonly used wavefunction takes the form $\exp \left(i k_{x} x\right)$ instead of the form $\sin k_{x} x$ and is said to obey periodic boundary conditions. This exponential form only allows $k_{x}$ to take on integer values of $2 \pi / L_{x}$ instead of $\pi / L_{x}$, but compensates for the lower density of states by allowing negative values of $k_{x}$. This form gives totally erroneous results for the quantum well states and energies. Furthermore, it can be shown that Bloch states are no lenger a strictly valid concept. This will be discussed in Chapter IV, where the band theory is reworked.

Each staie has an energy given by equation (II.2) as

$$
E_{n}=\frac{\hbar^{2}}{2 m}\left(n k_{q}\right)^{2}=n^{2} E_{q}
$$

where $k_{q}$ and $E_{q}$ are defined by

$$
k_{q} \equiv \frac{\pi}{L_{z}}
$$

and

$$
E_{q} \equiv \frac{\hbar^{2}}{2 m} k_{q}^{2}
$$




\section{Quantum Well Density of States}

This quantization of states from an infinite barrier is now seen to lead to quantized energy states increasing in energy in proportion to the square of the quantum state number. But in a three dimensional crystal confined in one dimension as above, the particles are free to move in the lateral or trarsverse dimensions. This freedom allows the particles to take on a continuum of energies, and the energy density of states to take on a stairstep appearance. First, in three dimensions equation (II.3) takes on the separable form

$$
\psi_{\text {env }}(\vec{r}, t)=X(z) Y(y) u(z) e^{-i E t / \hbar}
$$

as a result of the separability of Schrödinger's equation, (II.1), with the quantum well potential varying only in one dimension. The $x$ and $y$ dimensions have boundary conditions similar to that of the $z$ dimension. In particular the wavefunction must go to zero at the $x$ and $y$ boundries and be zero outside the crystal. However, the dimensions of crystals of interest in the $x$ and $y$ directions are macroscopic and individual states are sufficiently close in energy to be indistinquishable. As such we can use wavefunctions, as is standard in solid state theory, oi the form $\exp \left(i k_{x} x\right)$ and $\exp \left(i k_{y} y\right)$ for the $\mathrm{x}$ and $\mathrm{y}$ dimensions. Also customary for this form of the wavefunctions, we use the periodic boundary conditions. Assume the crystal has transverse dimensions $L_{x}$ and $L_{y}$. Then the allowed wavevectors are evenly spaced in $k$ space with a spacing of $2 \pi / L_{x}$ and $2 \pi / L_{y}$, respectively. Now define a trancverse wavevector $k_{t}$ by

$$
k_{t} \equiv \sqrt{k_{x}^{2}+k_{y}^{2}}
$$

The number of states with an absolute value of the wavevector between $k_{t}$ and $k_{t}+d k_{t}$, where $d k_{t}$ is much smaller than $k_{t}$, is then given by $\left(L_{x} L_{y} / 4 \pi^{2}\right) 2 \pi k_{t} d k_{t}$. For 
a particle in free space with constant poisntial, the relation between energy and wavevector in the two transverse dimensions is given by

$$
E_{t}=\frac{\hbar^{2}}{2 m} k_{t}^{2}
$$

Taking the derivative, this equation becomes

$$
d E_{t}=\frac{\hbar^{2}}{m} k_{t} d k_{t}
$$

The number of states with energy between $E_{t}$ and $E_{t}+d E_{t}$ per unit area, $\rho\left(E_{t}\right)$, is then given. by equation (II.10) and the above relation for density in $k_{t}$ space. If in addition the particle has a spin degeneracy $g_{0}$,

$$
\rho\left(E_{t}\right) d E_{t}=\frac{g_{s} m}{2 \pi \hbar^{2}} d E_{t}
$$

and we soe that the density of states does not vary with $E_{t}$ for a given quantum state.

This derivation ior density of states per unit area has made several assumptions. First and most importantly, equation (II.11) applies to each quantum state. Thus each quantum state has a density of states given by equation (II.11). Second, since $E_{t}$ must be positive, from equation (II.9), no states exist for $E_{t}$ less ti..n zero. This means

$$
\rho\left(E_{t}\right)=0, \quad E_{t}<0 .
$$

If a step function is defined by

$$
\operatorname{Step}(x)=0 \quad x \leq 0
$$


and

$$
\operatorname{Step}(x)=1 \quad x>0
$$

then the density of states can be extended to negative values of $E_{t}$ by the formula

$$
\rho_{l}\left(E_{t}\right) d E_{t}=\frac{g_{8} m}{2 \pi \hbar^{2}} \times \operatorname{Step}\left(E_{t}\right) d E_{t}
$$

From the use of equation (II.7) in equation (II.1), we have the total energy $E$ given by

$$
E=E_{\mathrm{l}}+n^{2} E_{q}
$$

The density of states as a function of total energy is now given by the summation over all quantum states with use of equations (II.14) and (II.15):

$$
\rho(E) d E=\sum_{n=1}^{\infty} \rho_{l}\left(E-n^{2} E_{q}\right) d E=\sum_{n=1}^{\infty} \frac{g_{8} m}{2 \pi \hbar^{2}} S t e p\left(E-n^{2} E_{q}\right) d E
$$

which is illustrated in Fig. II.1. As the quantum number $n$ becomes large compared to unity, it can be seen that $\rho(E)$ is proportional to the square root of $E$, as is expected for a particle in a three dimensional box.

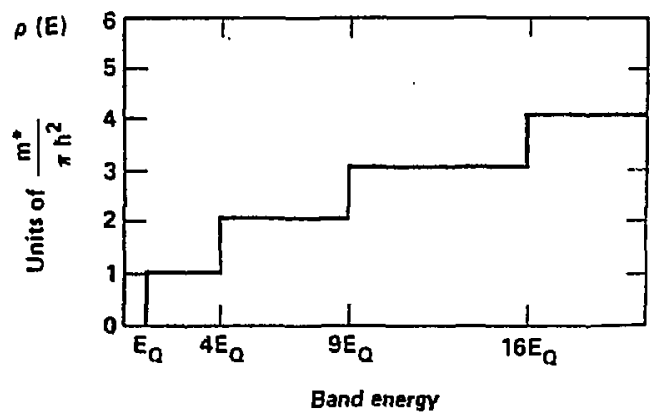

Figure II.1: A plot of the density of states vs. electron energy for a quantum well. 


\section{Two-Dimensional Excitons}

Solid state physics has long recognized and observed excitons in semiconductors. Excitons are composed of an electron in the conduction band and a hole in the valence band bound to each other by a coulomb potential. The solution in three dimensional space is very similar to the solution for the hydrogen atom in free space, but with a larger dielectric constant and a smaller electron mass. The justification for this approximation is tedius and given in the large amount of literature on the exciton.(10,11] The energy is thus given by

$$
E_{e x}(n)=-\frac{m_{r}^{*} e^{4}}{2 \hbar^{2} \epsilon^{2} n^{2}}
$$

in CGS units, where $m_{r}^{*}$ is the effective reduced mass (see equation II.28 for definition), $e$ is the electron charge, and $\epsilon$ is the dielectric constant. For GaAs, with $m_{r}^{*}$ about 0.065 electron masses and $\epsilon$ around $11, E_{e x}(n)$ is approximately $7.5 \mathrm{meV} / \mathrm{n}^{2}$. The radius $a_{e x}$ of the exciton is analogous to the Bohr radius of the hydrogen atom and is given by

$$
a_{e x}=\frac{\hbar^{2} \epsilon}{m_{r}^{*} e^{2}}
$$

and is about $90 \AA$ for GaAs. The diameter is then approximately $180 \AA$.

This three dimensional model of the exciton is based on the electron and hole being confined by only their mutual attraction. But when the confining boundries of the particles are separated by distances smaller than the exciton diameter this model is obviously no longer valid. The solution for the two dimensional case has been given by Ralph[12] and a derivation is outlined here. For sufficiently small confinement in one dimension, the electron wavefunction can be broken into a product of the previous quantum well states in equation (II.4) and a two dimensional 
wavefunction as in equation (II.7). This two dimensional wayefunction is solved by a two dimensional Schrödinger equation (equation (II.l) in two dimensions). Let the wavefunction $u(r, \phi)$ be the wavefunction in cylindrical coordinates $r$ and $\phi$ which is separable into the form

$$
u(r, \phi)=A R(r) \Phi(\phi)
$$

This wavefunction obeys the two dimensionsl Schrödinger equation in cylindrical coordinates of the form

$$
-\frac{\hbar^{2}}{2 m_{r}}\left[\frac{1}{r} \frac{\partial}{\partial r}\left(r \frac{\partial u}{\partial r}\right)+\frac{1}{r^{2}} \frac{\partial^{2} u}{\partial \phi^{2}}\right]-\frac{\epsilon^{2}}{\epsilon r}=E u .
$$

The separable equation for $\Phi$ is easily solved to give

$$
\Phi(\phi)=e^{i m \phi}
$$

where $m$ is an integer. Now make the following definitions

$$
\begin{gathered}
\rho \equiv \alpha_{n} r \\
\alpha_{n}^{2} \equiv-\frac{8 m_{r} E_{n}}{\hbar^{2}} \\
\lambda_{n} \equiv \frac{e^{2}}{\epsilon h}\left(-\frac{m_{r}}{2 E_{n}}\right)^{1 / 2}
\end{gathered}
$$

and

$$
R(r) \equiv e^{-\rho / 2} F(\rho)
$$


With these definitions, equation (II.20) becomes

$$
F^{\prime \prime}+\left(\frac{1}{\rho}-1\right) F^{\prime}+\left[\frac{\lambda_{n}-1 / 2}{\rho}-\frac{m^{2}}{\rho^{2}}\right] F=0
$$

where the prime on $F(\rho)$ indicates a derivative with respect to $\rho$. The function $F(\rho)$ can be solved by a polynomial expansion in $\rho$ but remains finite as $\rho$ goes to both zero and infinity only if $\lambda$ takes on values of $n+\frac{1}{2}$ where $n=0,1,2, \ldots$ The energy $E_{n}$ is now given by equation (П1.24) and takes on values $[12]$

$$
E_{n}=-\frac{m_{r} e^{4}}{2 \hbar^{2} \epsilon^{2}} \frac{1}{(n+1 / 2)^{2}}
$$

On comparison with equation (II.17), we find the maximum binding energy of the two dimensional exciton is four times larger than the three dimensional exciton. In GaAs, this energy is then about $\mathbf{3 0} \mathrm{meV}$. This large increase in binding energy has been used, with significant advantage, to increase the fraction of the electron-hole population residing in the exciton state at room temperature.|5|

When the confining boundries in one dimension are no longer small compared to the exciton diameter, the two dimensional approximation breaks down. However, we also know the solution in the other limit of large boundaries is simply that of the three dimensional exciton. The interpolation between the two is difficult analytically and is not known to be solved. Unfortunately, the dimensions of the quantum wells of interest are in the intermediate regime. As such the energies of these excitons are closer to the three dimensional exciton energies and would be expected to be about 2 to 3 times the three dimensional exciton energies or about 15 to $20 \mathrm{meV}$. Furthermore, the spectra of the excitons indicate that the above eivergies are high by a factor of two, so that the actual three dimensional binding energies are at - nt 4 meV and the quantum well excitons of 5 to 10 nanometers thickness have about 10 
meV binding energies. $[3,5]$ A possible reason for the smaller energy is that the reduced mass of the exciton was taken to be that of the electron in the conduction band. But the valance band has a mass which varies from $0.5 \mathrm{~m}^{*}$ to $0.082 \mathrm{~m}^{*}$, depending on which of the three valance bands is chosen. Thus the reduced mass will be somewhere between $0.058 m^{*}$ and 0.036 , with the actual value to be found from a more detailed analysis of the exciton effect. However, it is seen from equation (II.17) that this lowered reduced mass yields a lower exciton binding energy and gives better agreement with observation. Unlike interband spectra, excitons are not of interest to the QWEST abservations because the QWEST operates entirely in the conduction band and never creates the holes in the valence band needed for an exciton. So no excitons ever exist for the type of measurements performed. 


\section{E. Interband Spectra}

A large number of experiments have been perfor med to investigate the properties of optical transitions between the conduction and valence bands in GaAs quantum wells..$^{[3,13,14]}$ In particular, the absorption spectra ${ }^{[3]}$ and photoexcitation spectra ${ }^{[13]}$ have been measured in quantum well samples. The photoexcitation experiment scans a pump laser beam in frequency above the bandgap edge while observing the spontaneous emission of light at the bandgap energy. The assumption is that relaxation within the band of the electron and hole are fast enough compared to the spontaneous emission rate that all transitions emit at the bottom of the energy bands. Thus the emission rate at this band edge reflects the strength of the original absorption but is free of the background light of the pump laser with adequate filtering of the light spectrum.

This conduction-to-valance band, or interband, sprectrum can be derived from the discussions of the previous sections. First, for the free electron and hole the envelope state is described by equations (II.4) and (II.7). The optical dipole is between the Bloch states which multiply these envelope states to completely specify the particle wavefunction. The Bloch states vary slowly with energies of interest here (to be shown in a later chapter), so the transition strength is also nearly constant with transition energy. In order for the transition to have a nonzero dipole, and therefore nonzero strength, the envelope states for the electron and hole must have the same quantum well number and the same transverse wavevector, $k_{\mathfrak{t}}$, since these states are orthogonal. Although the photon can contribute some momentum to the wavevector, the contribution is iusignificant in comparison with the electron's much larger momentum. The energy difference for a particular transition can then be given by equation (II.15) with $m$ in equations (II.6b), (II.8), (II.11), and elsewhere replaced by $m_{r}^{*}$, where, as in equation (II.17), 


$$
\frac{1}{m_{r}^{*}}=\frac{1}{m_{e}^{*}}+\frac{1}{m_{h}^{*}}
$$

and $m_{e}^{*}$ is the effective electron mass and $m_{h}^{*}$ is the effective hole mass. The density of states for transition energy is then giver by equation (II.16) with $m=m_{r}^{*}$. Finally, let the wells be undoped and at low temperature so the valence band is full and the conduction band is empty. Since the transition strength and occupation probability are constant for each of these transitions, the free electron and hole absorption spectrum is given by transition density density of states, or the shape of Fig. Il.1 with $m=m_{r}^{*}$.

For each quantum well state $u_{n}(z)$, a two dimensional exciton exists which has a quantized energy, given by equation (II.27). This energy, resulting from the transv srse degrees of freedom and the coulomb interaction of the electron and hole, adds to the quantum well transition energy in equation (II.5) with $m=m_{r}^{*}$. Since this energy is negative, a peak in the absorption spectrum occurs in front of each step of Fig. II.1, giving a experimental absorption spectrum shewn in Fig. II.2. The resonance of the exciton has some energy spreading from such mechanisms as phonon dephasing and irregularities in quantum well thickness. [5]

The valance band consists of three different bands. One band is split off with a different energy ai zero transverse wavevector. The other two are degenerate at zero wavevector, but have different effective masses. Each of these bands has an absorption spectra, with the value of $m_{r}^{*}$ different for each of the bands. The overall interband absorption spectra is the sum of the spectrum for each of the three valance-to-conduction band transitions as described above. The split-off band has an energy $0.34 \mathrm{eV}$ below the degenerate bands and is not in the spectral region of most interband spectra. The degenerate valance band creates two valance quantum wells for each wavefunction given in equation (II.4). For each quantum number, n, 
there exists two different quantum energies, given by the different effective masses of the degenerate bands used in equations (II.5) and (II.6). This energy difference is slight but the two states can be resolved on higher resolution spectra.[13]

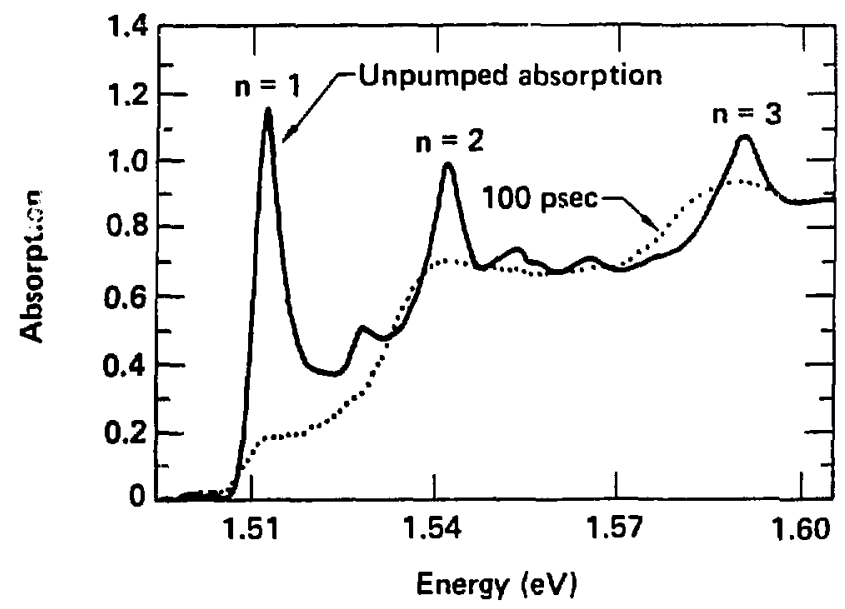

Figure II.2: Absorption spectra of a GaAs quantum well at $77 \mathrm{~K}$ both before and 100 picoseconds after pumping to an electron density of $5 \times 10^{11} \mathrm{~cm}^{-2}$. Note that the excitons disappear with the presence of the free electrons because of the shielding of the coulomb potential (from Ref.[27]). 


\section{F.Inelastic light scattering}

The quantum wells have also been probed by inelastic light scattering.|18,11] In the typical experiment, light is incident normal to the sample with a photon energy equal to the sum of the band gap energy, $E_{0}$, and the split off valance band energy, $\Delta_{o}$, so as to be in resonance between the conduction band and the lower energy split-off valance band (see Fig. II.3.). The photon causes an electon in the split-off valance band to be injerted into the conduction band with the same transverse wavevector as in the initial valance state, as discussed above for interband transitions. However, for the inelastic light scattering, the conduction band is populated and a conduction band electron with the same transverse wavevector, but in a smalller energy quantum well state, falls into the recently vacated valance band hole, emitting a photon in the process. Since the transverse wavevector remains constant, the emitted photon would be the same energy as the absorbed photon, giving elastic scattering, if the quantum well state does not change quantum number. But the transitions of interest are the inelastic transitions, in which the conduction band quantum well states are not the same for the initial and final states. In this case, the energy difference of quantum well states can be found from the energy difference of the absorbed and scattered photon. The band gap energy can therefore be subtracted out. These interband transitions use a dipole between the Bloch states, and the integral of the product of the initial and final envelope states multiplies this integral. The quantum well envelope states are orthogonal for an infinite barrier potential and thus no change in quantum well envelope state sould be allowed were it not for finite barrier giving slightly different, and not quite orthogonal, envelope states for the conduction and valance bands. 


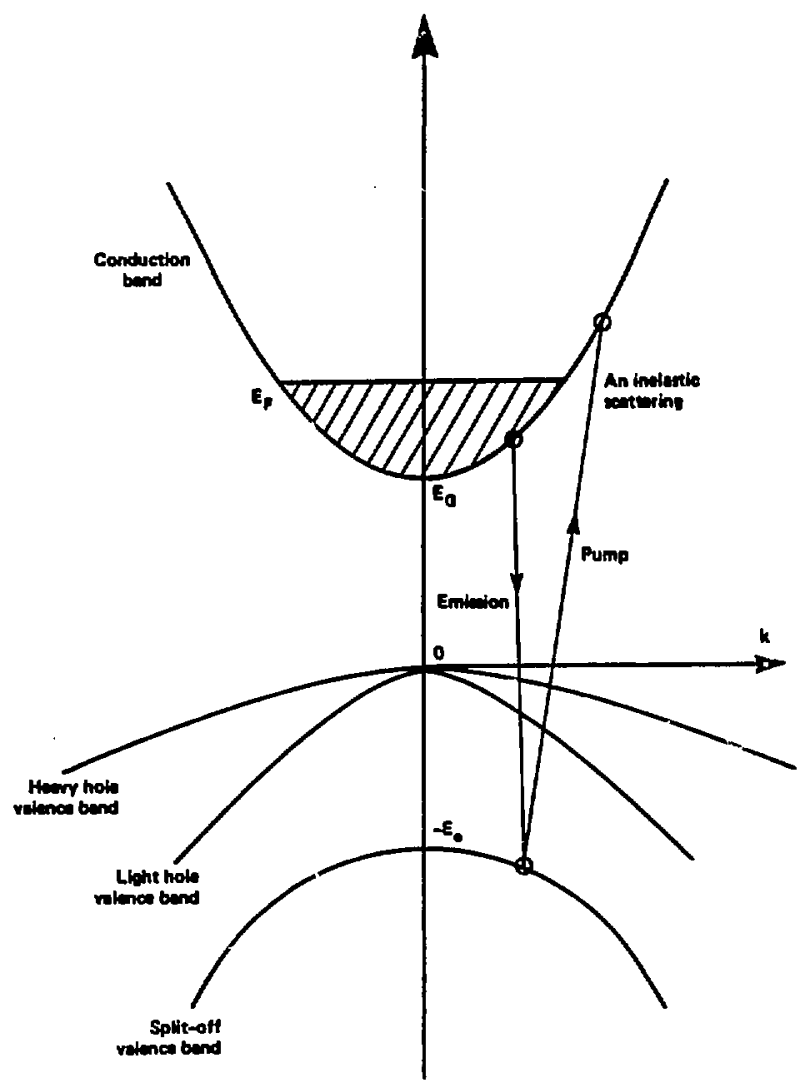

Figure II.3: A plot of the conduction and valence bands of GaAs, including a possible inelastic scattering.

Furthermore, the incident photon to be absorbed selects out a narrow range of wavevector states, thus the quantum well energy difference can be measured as a function of transverse wavevector and the emitted photon spectra is narrow. The narrow photon emission spectra is obtained by the selection of a narrow range of transverse wavevector states by the incident photon. In contrast, the exciton 
spectra is narrow because of the creation of quantum states without free electrons in any dimension, thus creating a state with only one energy. In the QWEST to be discussed, not only are the electrons free in two dimensions, leading to broad energy states, but any of these states can make a transition. The QWEST nevertheless is seen to be narrow because all these transition energies happen to be the same.

The split-off valance band is shifted from the other two valance bands by spinorbit coupling. This same interaction also quantizes this split-off valance band's Bloch state into a sum of two wavefuctions, $\phi_{v}=(2 / 3)^{1 / 2}(X+i \gamma) / \sqrt{2} \downarrow+(1 / 3)^{1 / 2} Z$ , where the arrow refers to the electron spin and $X, Y$, and $Z$ refer to the same odd symmetry valance wavefunction, when rotated to be symmetric about either the $\mathrm{x}, \mathrm{y}$, or $\mathrm{z}$ directions respectively. These wavefunctions will be derived in a later section. The conduction band's single Bloch state, labelled $s$, is rotationally symmetric and of course can have either electron spin. A dipole exists between the $S$ state and any of the $X, Y$, or $Z$ states and gives rise to the conduction to valance band transitions. Now let the quantum well be confined in the y direction, in contrast to the $z$ direction as in equations (II.1) through (II.6), and the electron is now free in the $x$ and $z$ direction. Now assume an electron is optically excited from the $\mathrm{n}_{\mathrm{i}}=1$ envelope state in the split-off valance band into the $n=2$ envelope state in the conduction band by light polarized in the $x$ direction and propagating in the $y$ direction, normal to the quantum well surface. Then given the form of $\phi_{v}$ above we see that the conduction band electron must have a $\downarrow$ spin. The hole in the valance band can now be filled by an electron in the conduction band's $n=1$ envelope state. If this conduction band electron's Bloch state has a $\downarrow$ spin, as did the electron excited to the $n=2$ conduction envelope state, then we see from the form of $\phi_{v}$ that the dipole is in the $\mathbf{x}$ direction and emits light with this polarization. If, on the other hand, the electron filling the valance band hole has a 1 spin, the dipole is seen to 
be in the $z$ direction, with orthogonal polarization to the original inciuent beam. Thus the inelastic scattering transfers an electron from the $m=1$ conduction band envelope state to the $m=2$ conduction band envelope state. The emitted spectra with a polarization parallel to the incident spectra results from a transition with no change in electron spin, and the emitted spectra witb a polarization perpendicular to the incident light polarization results from a transition in which the eleciron's spin tlips.

The ability to probe transitions with and without spin flip is used to separate collective electron effects from single particle effects. If the electron changes spin in the transition, the excited state cannot interact with the remainder of the electron gas, which is Fermi degenerate with an opposite spin hole, because of Fermi repulsion. In contrast, if the electron does not change spin, the excited electron interacts with the remaining conduction band electron plasma and has a different excitation energy than that of the noninteracting single particle excitation. The collective excitation has a higher absorption energy than the single particle excitation because of the extra energy in the oscillating polarization field of the oscillating charge coherence between the lower and upper envelope statis. The absorption erergy of the collective effect, $\omega_{12}^{*}$, is given in terms of the quantum energy difference between the states, $\omega_{12}$, and the plasma frequency, $\Omega_{p}$, of the conduction band electron gas by the relation[32-84]

$$
\omega_{12}^{*}=\omega_{12}^{2}+\Omega_{p}^{2}
$$

The plasma frequency is close to the normal three dimensional plasma frequency obtained when the two dimensional electron density, $N_{6}$, is converted into a three dimensional electron density by dividing $w$ y the quantum well width, or $\Omega_{p}^{2} \sim$ $N_{0} e^{2} / m^{*} \epsilon L_{w}$. The above relation and the formula for the plasma frequency are 
developed later.

The experimental observations ${ }^{[17]}$ were performed at temperatures of $2 \mathrm{~K}$ and conduction band doping of about $5 \times 10^{11} \mathrm{~cm}^{-2}$. The quantum well widths used in this $\left|{ }^{17}\right|$ study were in the range of $250 \AA$. Therefore the lowest energy $\left(\Psi_{1} \rightarrow \Psi_{2}\right)$ quantum well transition has an energy of about $20 \mathrm{meV}$. The plasma frequency at this doping density is about the same as the transition resonant frequency. Furthermore, the phonons also have reson:ant frequencies in this range. Thus, although the single particle spin-flip transition has an energy shift at the $20 \mathrm{meV}$ energy, the collective mode spectra shows the energy shift as being higher as indicated in equation (II.29) above, with an energy of about $34 \mathrm{meV}$. Furthermore, the interaction of the phonons with the dielectric constant further split this non spin-flip spectra into two inelastic scattering energy shifts of about 28 and $39 \mathrm{meV}$. The spin-flip spectra, however, continues to exhibit only one unaffected scattered energy shifi at the quantum well resonant energy. The shifts caused by the collective effects will be shown to operate on the QWEST also, but be nearly unobservable because of the higher energy, over $100 \mathrm{meV}$, of the transitions with the much smaller well widths, 60 - $80 \AA$, used for the investigations of the QWEST. 


\section{G. Intersubband Transitions in Other 2-D Systems}

The nost similar 2-D transition to the QWEST are the infrared transitions occuring between the surface charge states at the top surfaces of $\mathrm{Si}^{[37]}, \operatorname{InAs}{ }^{[38 \mid}$, InSb[30), and ( $\mathrm{HgCd}) \mathrm{Te}^{[10]}$. These observations were all reported by F. Kocì and his group at Technische Universität München in West Germany over the las several years. The surface charge states are electrons which are trapped at the surface of a semiconductor. With an sppropiate cover, the band will bend down within about $100 \AA$ of the surface and the electrons will lie within quantized states at this boundary. The energy of these states can be changed by application of 9 , voliage across the interface and thus bending the bands. Typically the electron suriace densities are in the $1 \times 10^{12} \mathrm{~cm}^{-2}$ range and the energies of the transition lie in the 30 to $100 \mathrm{meV}$ range. These transitions were observed by illumination with a far infrared laser while changing the voltage across the interface.

These transitions are similar in nature to the QWEST, but differ in several aspects. First, the bands are not $\%$ mmetric, thue even parity transitions become allowed. Second, the transitions and surface density are very itrongly coupled, as the electrons themselves perform the confinement on one side of the surface layer. The QWEST can entirely decouple these quantities. $n$. t the scan of the surface charge transition as a function of voltage makes measurement of the absorption strength difficult as the ciensity changes significantly ( $30 \%$ ) from olie side of the absorption to the other. Third, the QWEST is capable of transition energies of over $500 \mathrm{meV}$. Energies this high, if even possible, from surface charge layers would require surface charge densities so high that the transition would be very broadened. Fourth, the band bending is very dependant on the tem perature for a given charge density, and the energy of the transition changes very dramatically with temperature. In contrast, the QWEST will be seen to change very little $<<$ 
$3 \%$ ) over a large temperature range $(30-300 \mathrm{~K})$.

These are only a few of the differences between these quantum wells and surface charge layers. Yet another way to create surface charge layers in semiconductors is by aiternating layers of $\mathbf{n}$ and $\mathbf{p}$ type materials. Such stuctures are called NIPI layers. (1) These layers are confined on both sides by the potential set up by the charges themselves. The discussion above for surface charge layers also apply to NIPI layers as they are confined on both sides by tive same sort of self-coulomb potential that exists on one side for surface charge layers. No obser vation has yet been reported for infrared transitions between the envelope states of the NIPI layers.

Another related development involves free carrier photoionization of electrons out of GaAlAs quantum well states. A photodetector based on this effect has been proposed recently by D. D. Coon and R. P. G. Karunasiri at University of Pittsburgh. ${ }^{103]}$ A device with much lower absorption strength was proposed and tested by J. S. Smith, L. C. Chui, S. Margalit, A. Yariv, and A. Y. Cho at Caltech and Bell Labs. ${ }^{(61)}$ Their devicelo4] had an absorption of about $30 \mathrm{~cm}^{-1}$ and was used to create a photodetector with a response of 200 Amps per Watt of infrared power. 


\section{III.Infinite Barrier and Parabolic Energy Bands}

\section{A. The Envelope State Transition}

The energy and wavefunction of a three dimensional particle trapped beiween two coplaner barriers was considered in chapters II.B. and II.C.. The density of states per unit of energy was also derived. These results are well known to any beginning quantum mechanics student but nevertheless give accurate predictions of GaAs quantum well properties. ${ }^{(3,4)}$ These expressions will now be used to develop properties of Quantum Well Envelope State Transition. The states in equation (II.7) are called envelope states because they are not the full electron state in GaAs. The full electron state must include the wavefunction describing the local properties about each atom in the crystal lattice. But, as will be shown later, the behavior of the GaAs transition under consideration is determined almost entirely by the envelope states.

From equation (II.7) the wavefunction for the envelope state is given by

$$
\psi_{\text {env }}\left(k_{x}, k_{y}, n\right)=\sqrt{\frac{2}{L_{x} L_{y} L_{z}}} e^{i k_{z} x} e^{i k_{y} y} \sin n \frac{\pi}{L_{z}}\left(z+L_{z} / 2\right) .
$$

This wavefunction does not include the time dependance which is given, for the energy eigenstates under consideration, simply by multiplying the above equation by $\exp (-i E t / \hbar)$ for a state with energy $E$. This energy, for the above states, is assumed to be of the same form as in equations $\Pi .5$ through II.15, namely

$$
E\left(k_{x}, k_{y}, n\right)=\frac{\hbar^{2}}{2 m^{*}}\left(n^{2} k_{q}^{2}+k_{t}^{2}\right)
$$

where, as before, $k_{q} \equiv \pi / L_{z}$ and $k_{t} \equiv \sqrt{k_{x}^{2}+k_{y}^{2}}$. The effects of the GaAs lattice potential are assumed to be taken into account by the effective mass, $m^{*}$. Thus, the energy of the electron in the conduction band has the same quadratic dependance 
on the wavevector, $k$, as would a free electron, only with a greatly reduced mass. This assumption is good, but the higher order dependance of the energy on the wavevector is important and will be discussed later.

The Quantum Well Envelope State Transition (QWEST) is an infrared dipole transition between two of the above quantum well states. In a (GaAl)As crystal, both initial and final states are in the conduction band for this transition. Although the electron envelope wavefunction of equation (III.1) is not the full $\mathrm{GaAs}$ wavefunction, the properties of the QWEST are determined almost entirely from this envelope wavefunction. As such, this wavefunction is used to predict the properties of the QWEST to first order. The corrections to this approximation are discussed later.

\section{B. The QWEST Dipole}

The QWEST involves the interaction of an infrared beam of light with discrete quantized states. This interaction between light and matter is treated in semiclassical quantum mechanics by the potential term in Schrödinger's equation, eq. (II.1). The semiclassical treatment is valid for cases here with the potential term $v(\vec{r})$ in equation (II.1) becoming $V(\vec{r})=V_{o}(\vec{r})+e \vec{r} \cdot \vec{E}(\vec{r}, t) . V_{o}(\vec{r})$ is the static potential describing the original problem, which has aiready been solved to give a particle wavefunction, such as equation (III.1) above. Here $e$ is the electron charge, and $\vec{E}(\vec{r}, t)$ is the electric field of the optical wave. The equation of motion resulting from inserting this new potential in Schrödinger's equation, (II.1), can be solved by time dependant perturbation theory, $[15]$ where the $\vec{e} \vec{r} \cdot \vec{E}(\vec{r}, t)$ term is the perturbation. Now assume the electric field is given by $\vec{E}(\vec{F}, t)=\vec{E}_{o} \exp \left(\vec{i}_{p} \cdot \vec{F}-i \omega t\right)$ The important parameter for the optical transition between two quantum mechanical states becomes the dipole matrix element between the two states of the perturbating potential. This matrix element, $\mu$, for the optical transition is given by 


$$
\begin{aligned}
\mu & =e\left\langle\psi_{f}\left|\vec{\gamma} e^{i k_{p} \cdot \vec{r}}\right| \psi_{i}\right\rangle \\
& =e \int \psi_{f} \vec{F} e^{i k_{p} \cdot \vec{r}} \psi_{i} d^{3} r,
\end{aligned}
$$

where $\psi_{f}$ and $\psi_{i}$ are the final and initial quantum states of the optical transition. The final and initial states ior the QWEST will be $\psi_{\text {env }}\left(k_{x}^{\prime}, k_{y}^{\prime}, m\right)$ and $\psi_{e n v}\left(k_{x}, k_{y}, n\right)$, respectively.

We now evaluate this dipole for the QWEST in the above approximations of infinite barriers and parabolic energy bands. From equation (III.1) we have the wavefunction of the two states. In performing the integral of equation (III.3) we find the integral is separable into three parts, labeled $X, Y$, and $Z$, which are different for each of the three vector components. We first consider the $z$ component of the dipole matrix element. We find

$$
\mu_{z}=e X Y Z
$$

where

$$
\begin{aligned}
& X=\frac{1}{L_{x}} \int_{0}^{L_{x}} e^{-i k_{x}^{\prime} x} e^{i k_{p x} x} e^{i k_{x} x} d x, \\
& Y=\frac{1}{L_{y}} \int_{0}^{L_{y}} e^{-i k_{y}^{\prime} y} e^{i k_{p y} y} e^{i k_{y} y} d y,
\end{aligned}
$$

and

$$
z=\frac{2}{L_{z}} \int_{\frac{-L_{z}}{2}}^{\frac{L_{z}}{2}} \sin m \frac{\pi}{L_{z}}\left(z+L_{z} / 2\right) e^{i k_{p z} z} z \sin n \frac{\pi}{L_{z}}\left(z+L_{z} / 2\right) d z .
$$

The values of $k_{x} L_{x}$ and $k_{y} L_{y}$ for cases of interest here are in the range of $10^{6}$ to $10^{8}$. It is seen that the integrals of equations (III.4b) and (III.4c) are then very small and equal to the inverse of the previously stated values unless $k_{x}^{\prime}=k_{p x}+k_{x}$ and 
$k_{y}^{\prime}=k_{p y}+k_{y}$, in which case they are equal to unity. As such, the transition will invariably satisfy these conditions. We next look at equation (III.4d), which contains the $z$ component of the dipole. We first note that the quantum wells of interest will generally have dimensions, $L_{z}$, less than 10 nanometers. The energies of the transition, to be determined from equation (III.2), will generally require resonances with infrared beams from 4 to 10 micrometers in wavelength, $\lambda$. The term in the exponent in equation (III.4d) will vary from 0 to $k_{p z} L_{z}=2 \pi L_{z} / \lambda$, which is seen to have a maximum value of $\mathbf{0 . 0 1 5}$, and is generally less. The exponential will therefore be very close to unity throughout the integral and can be ignored. The dipole integral of equation (111.4a) is now seen to reduce to

$$
\mu_{z}=e\left(\psi_{f}|z| \psi_{i}\right\rangle_{z}
$$

where

$$
e\left(\psi_{f}|z| \dot{\psi}_{i}\right\rangle_{z}=e \int_{0}^{L_{z}} \sin \left(m \frac{\pi}{L_{z}} z\right) z \sin \left(n \frac{\pi}{L_{z}} z\right) d z
$$

Note a change in the origin of the coordinate $z$ has been made. The integrals in $x$ and $y$ coordinates were seen above to drop out with the creation of a selection rule on the transverse wavevector of the final state, in terms of the transverse wavevector of the initial state and the electric field.

The dipole matrix element, $\mu_{z}$, of equation (III.5) will now be solved. We first note the comutation relation for the position operator, $\hat{z}$, with the Hamiltonion operator, $\hat{H}_{a}$, is given by the position operator:

$$
\left[\hat{H}_{0}, z\right]=-\hbar^{2} / m^{*} \frac{\partial}{\partial z}=-\frac{i \hbar}{m^{*}} \hat{p}_{z}
$$

where

$$
\hat{H}_{o}=-\frac{\hbar^{2}}{2 m^{*}} \frac{\partial^{2}}{\partial z^{2}}
$$


and

$$
\hat{p}_{z}=\frac{\hbar}{i} \frac{\partial}{\partial z}
$$

Similar relations can be found for the coordinates $\mathbf{x}$ and $\mathbf{y}$. This relation can now be used in the expression III. 5 to give

$$
\begin{aligned}
\left\langle\psi_{f}\left|\hat{p}_{z}\right| \psi_{i}\right\rangle & =\frac{i m^{*}}{\hbar}\left\langle\psi_{f} \| \hat{H}_{o}, z|| \psi_{i}\right\rangle \\
& =\frac{i m^{*}}{\hbar}\left(E_{m}-E_{n}\right)\left\langle\psi_{f}|z| \psi_{i}\right\rangle \\
& =\frac{i \hbar k_{q}^{2}}{2}\left(m^{2}-n^{2}\right)\left\langle\psi_{j}|z| \psi_{i}\right\rangle,
\end{aligned}
$$

where use has been made of equations (II.1) through (II.6). The evaluation of $\left\langle\hat{p}_{z}\right\rangle$ is straightforward. The sines in equation (III.5) are first converted into sums of complex exponentials. The operator $\hat{p}_{z}$ is then an eigenvalue of these exponentials. The resulting combinations of exponents must then be gathered and integrated over the integer number of half cycles. The result of this integration is

$$
\begin{aligned}
\left\langle\psi_{f}\left|\hat{\theta}_{z}\right| \psi_{i}\right\rangle & =\frac{2 i \hbar}{L_{z}} \frac{n m}{m^{2}-n^{2}}\left[(-1)^{n+m}-1\right] \\
& =-\frac{4 i \hbar}{L_{z}} \frac{n m}{m^{2}-n^{2}} \operatorname{Par}(n+m) .
\end{aligned}
$$

We have defined a function $\operatorname{Par}(n)$ as a function of any integer $\mathbf{n}$ which takes on the values

$$
\operatorname{Par}(n)= \begin{cases}0, & \text { if } n=\text { even; } \\ 1, & \text { if } n=\text { odd }\end{cases}
$$

Using equations (III.7) and (III.8) we finally obtain an expression for the dipole for an arbitrary quantum well envelope state transition:

$$
e\left(\psi_{f}|z| \psi_{i}\right\rangle=-e L_{z} \frac{8}{\pi^{2}} \frac{m n}{\left(m^{2}-n^{2}\right)^{2}} \operatorname{Par}(n+m) .
$$

This dipole is seen to exist, as expected, only for transitions between envelope states of opposite parity. The transition receiving most of our attention will be between 
states $m=2$ and $n=1$, or between the two lowest energy states. For this transition, the dipole is seen to be

$$
e(2|z| 1)=-e L_{z} \frac{16}{9 \pi^{2}} \approx-0.18 e L_{z}
$$

for a well of thickness $L_{2}$.

We now look at the other components of the dipole matrix element in the $\mathrm{x}$ and $y$ direction. Note first that the above matrix element made no assumptions about the size of $L_{z}$. Thus equation (III.10) applies to the other directions $\mathrm{x}$ and $\mathrm{y}$ simply by substitution of the appropiate dimension, $x$ or $y$, for $z$ in the equation. The principle observable differences arise from $L_{x}$ and $L_{y}$ being about $10^{\beta}$ times larger than $L_{z}$ for the samples of interest. From equation (III.2), the energy of a transition is lowered by the square of this well width and thus is about $10^{12}$ times smaller. These energies are not of interest and too easily broadened to be observable. In order for $a \mathrm{n}=1$ to $\mathrm{m}$ transition to have sufficient energy to be comparable with the quantum well energies, $\mathrm{m}$ must be as high as $L_{x} / L_{z} \sim 10^{6}$. But we see from equation (III.10) that the dipole matrix element is reduced to about $e L_{x} / 10^{18}$ or $e L_{z} / 10^{12}$ and thus is effectively zero at the energies of observation. The dipole matrix element for the QWEST is therfore polarized in the $\mathrm{z}$ direction with zero components in the other two directions.

\section{C.The QWEST Energy}

The energy of the envelope states is given in equation (III.2). In the previous section we found that dipole matrix element gave strong enhancement to final states with transverse wavevectors which are the vector sum of the initial envelope state transverse wavevector and the photon's transverse momentum vector. Using this selection rule, we find the transition energy from state $\psi_{\text {env }}\left(k_{x}, k_{y}, n\right)$ to state $\psi_{\text {env }}\left(k_{x}^{\prime}, k_{y}^{\prime}, m\right)$ as 


$$
\begin{aligned}
\Delta E\left(m, k_{t}^{\prime} ; n, k_{t}\right) & =\frac{\hbar^{2}}{2 m^{*}}\left[\left(m^{2}-n^{2}\right) k_{q}^{2}+k_{t}^{2}-k_{t}^{2}\right] \\
& =\frac{\hbar^{2}}{2 m^{*}}\left[\left(m^{2}-n^{2}\right) k_{q}^{2}+k_{p t} \cdot k_{t}+k_{p t}^{2}\right]
\end{aligned}
$$

where $k_{p t}$ is the component of the photon wavevector in the plane of the quantum well. As remarked previously, the value of $k_{p}$ is about a thousand times smaller than either $k_{q}$ or $k_{t}$. The above energy reduces to

$$
\begin{aligned}
\Delta E\left(m, k_{t}^{\prime} ; n, k_{t}\right) & =\frac{\hbar^{2} k_{q}^{2}}{2 m^{4}}\left(m^{2}-n^{2}\right) \\
& =\frac{\hbar^{2} \pi^{2}}{2 m^{*} L_{z}^{2}}\left(m^{2}-n^{2}\right)
\end{aligned}
$$

and becomes independent of the initial wavevector. This very important result indicates the density of states for the transtition is a delta function and has an infinitely narrow linewidth. This result is in stark contrast to the conduction-tovalance band transitions in which the transition density of states increases as the square root of $E-E_{G}$ for three dimensional crystals or a step function for two dimensional crystals as shown in section II.E..

D. The QWEST Oscillator Strength

We define an oscillator strength, $f$, for the transition between quantum well states $m$ and $\mathbf{n}$ by the formula,

$$
f \equiv \frac{2 m_{e} \omega}{\hbar}(z)^{2}
$$

where $m_{e}$ is the free electron mass, $\omega$ is equal to the energy difference between the states divided by $\hbar$, and $(z)$ is the dipole between the states as defined in equation (III.10) for the potential under consideration here. The above definition of the oscillator strength is based on the relative dielectric response of a quantum 
mechanical electron to a classical bound electron ${ }^{[0]}$ and is thus very general and will be used later in a more accurate theory of the QWEST in (AIGa)As quantum wells. This oscillator strength also obeys a sum rule[18] which for a single electron transition is given by

$$
\sum_{k} f_{0 k}=1
$$

where the sum is over all transitions which start from state $s$ to any and all other states, labeled $k$. The oscillator strength is positive when coupling to energies above $E_{8}$ and negative when coupling to lower energies. We observe that the derivation of this sum rule is sufficiently fundamental and general so as to also apply to our system. The derivation relys only on the comutation relations $\left[z, p_{z}\right]=i \hbar$ and $[H, z]=-i \hbar p_{z}$ which apply to all quantum mechanical systems. If we use the energy of a envelope state transition from equation (III.13) and the transition dipole from equation (III.10), we obtain an oscillator strength for a transition between quantum well states $m$ and $\mathbf{n}$ given by

$$
f_{m n}=\frac{m_{e}}{m^{4}} \frac{64}{\pi^{2}} \frac{m^{2} n^{2}}{\left(m^{2}-n^{2}\right)^{3}} .
$$

The oscillator strength has the interesting property of increasing linearly with $\mathrm{m}$ for a $(m \rightarrow m+1)$ transition. For a $\left(\Psi_{1} \rightarrow \Psi_{2}\right)$ transition, $f=0.96 m_{e} / m^{*}$. For a $\left(\Psi_{2} \rightarrow \Psi_{3}\right)$ transition, $f=1.87 m_{e} / m^{*}$. For GaAs $m_{e} / m^{*} \simeq 15$.

The sum of QWEST oscillator strengths between a given quantum state and all other states can be found by creating an artificial hamiltonian as we have done in the previous sections and deriving the sum rule using the effective mass of this system. The oscillator strengths must, as before, sum to unity. But if we renormalize the oscillator strengths to the free electron, we find that for the QWEST, $\Sigma_{m} f_{m n}=$ 
$m_{e} / m^{*}$. But in a real system, we know that the sum must continue to equal unity. Obviously, there are other transitions which must be included in a real system in the sum rule. We will see how this occurs in detail at the end of the next chapter. 
IV.Quantum Wells in (AlGa)As Crystals

This chapter is concerned with the structure of the QWEST in a real crystal such as the (GaAl)As compounds. A single quantum well would consist of a planer well region of GaAs with a thickness $L_{w}$ of about $100 \AA$ covered on both sides by infinite sheets of $\mathrm{Ga}_{1-\mathrm{x}} \mathrm{Al}_{\mathrm{x}}$ As (see Fig. IV.1). Throughout this chapter, we will take the zero of the $\mathrm{z}$ coordinate at the center of the well, so the bonndaries occur at $\mathrm{z}=$ $\pm L_{z} / 2$. We make this change so as to more easily take advantage of the symmetry properties of these wells. We placed the zero at the edge of boundary throughout the previous chapter because the infinite barrier caused the wavefunction to go to zero at the boundaries and allowed us to describe all envelope states by sine wave functions. By taking the zero at the center of the well, we have the additional complication of having to use cosine functions for even symmetry states and sine functions for odd symmetry states. We begin by looking at the properties of GaAs and their effects on the QWEST.

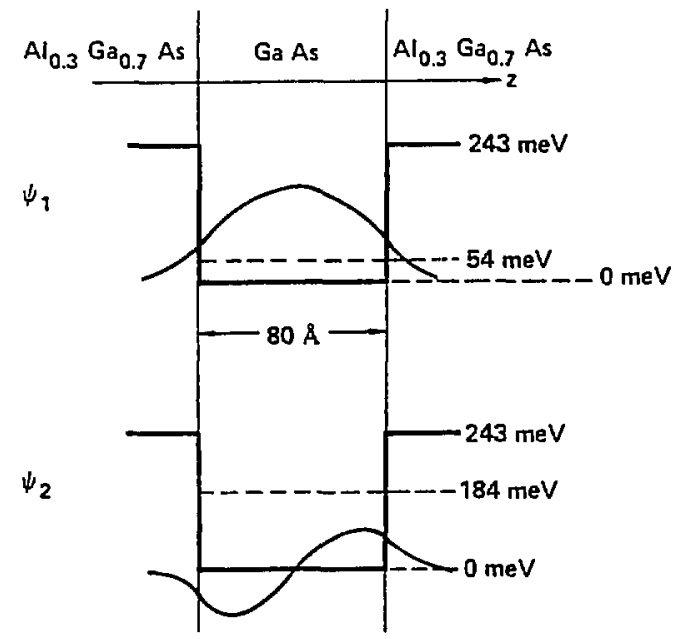

Figure IV.1: A diagram of an actual quantum well in (AlGa)As materials. The potentials and two lowest envelope wavefunctions are illustrated. 


\section{A. Band Structure in Gais}

In the previous chapter the states were labeled envelope states and the wavefuctions were labeled envelope wavefuctions. This distinction was made because the full condunction band electron wavefunction in (AJGa)As compounds is generally a product of this wavefunction and the Bloch wavefunction. The Bloch wavefunction describes the part which is periodic from one crystal unit cell to another. The envelope wavefunction describes the nonperiodic behavior. In contemporary solid state theory, the full wavefunction is taken to be a product of these two wavefuctions. This approximation will be seen to need modification for the quantum well states. However, within this approximation, the electron behevior can be well described by the envelope with its kinetic energy based on a lower effective mass as done in the previous chapter. In this chapter, we look more at the complete wavefuction and energy.

The important behavior of the GaAs conduction band, which is where the QWEST occurs in entirety, can be adequately described by $k \cdot p$ theory. ${ }^{[20]}$ The $k \cdot p$ theory is an expansion in $k \cdot p$ around any $\vec{k}$. Fortunately, the region of interest for our experiments is a small region in $\vec{k}$ space about $\vec{k}=0$. Because we will need much of the results and use many parts of the $k \cdot p$ theory in our development, we will sketch the major points in a relatively quick derivation.

We first note that solid state theorists generally use a wavefunction of the form

$$
\Psi(r)=\Psi_{e n v}(r) u(r)
$$

where $u(r)$ is the Bloch wavefuction and is periodic with the lattice spacing. The envelope wavefunction, $\Psi_{\text {env }}(r)$, describes the nonperiodic extended behavior of the electron wavefuction. For periodic boundry conditions in uniform crystals, $\Psi_{\text {env }}$ is generally shown to take on the form 


$$
\Psi_{\text {env }}=e^{i \vec{k} \cdot \vec{t}}
$$

The value of $\vec{k}$ is quantized by the periodic boundry conditions into taking on the values only of

$$
\vec{k}=n \frac{2 \pi}{L_{x}} \hat{z}+m \frac{2 \pi}{L_{y}} \hat{y}+p \frac{2 \pi}{L_{z}} \hat{z}
$$

where $\mathrm{n}, \mathrm{m}$, and $\mathrm{p}$ are positive and negative integers and $L_{x}, L_{y}$, and $L_{z}$ are the crystal's dimensions in the $x, y$, and $z$ direction. If we now apply Schrödinger's equati in to the wavefunction as given by equations (IV.1) and (IV.2), we find the Schrödinger equation for the Bloch state wavefunction becomes

$$
\begin{aligned}
\left\{p^{2} / 2 m+V+\right. & (\hbar / m) \vec{k} \cdot \vec{p}+\left(\hbar / 4 m^{2} c^{2}\right)[\nabla V \times \vec{p}] \cdot \sigma \\
& \left.+\left(\hbar^{2} / 4 m^{2} c^{2}\right)[\nabla V \times \vec{k}] \cdot \sigma\right\} u_{k}(r) \\
& =\left\{E_{k}-\left(\hbar^{2} / 2 m\right) k^{2}\right\} u_{k}(r),
\end{aligned}
$$

where $V$ is the potential and $\vec{p}$ is the momentum operator. We have used the product rule for derivitives and applied the operators the full wavefunction and afterwards divided out the exponential envelope wavefunction. We now assume knowledge of the solution of the $k=0$ Bloch state wavefunction and energy. From group theory, the conduction band Bloch wavefunction is shown to have symmetry properties similar to the atomic $\mathbf{s}$ state and is therefore labeled $\mathrm{S}$. The valance band Bloch functions are shown to have symmetries similar to the atomic $p$ wavefunctions, have three-fold degeneracy and are labeled $X, Y$, and $Z$, depending on which is antisymmetric in the $x, y$, or $z$ direction, respectively. Each of these states are twofold degenerate depending on electron spin. Thus we have assumed an exact soluticn to the above first two terms in the Schrödinger's equation (IV.4). The last spin-o bit term in terms of $\vec{k}$ is neglected relative to the fourth term, which gives the spin-orbit in terms of the mach larger $\vec{p}$. The third $\vec{k} \cdot \vec{p}$ term and the fourth, 
spin-orbit, term are then treated as a perturbation. The $\vec{k} \cdot \vec{p}$ term will be seen to lead to the small effective mass of GaAs and the spin-orbit, term will be seen to lead to the split-off valance band. We now let $\vec{k}$ be in the $z$ direction, $\vec{k}=k_{z} \hat{z}$. The two perturbing terms can be shown to couple only four of the above eight states if they are normalized in the basis $\mathrm{S}_{\mathfrak{l}}, \mathrm{Z} !,(\mathrm{X}$-iY $) \uparrow,(\mathrm{X}+\mathrm{iY}) \uparrow$, where the arrow indicates the direction of the electron spin. The other four states, with spins in each of the states opposite to those here, are independant of the first four but.interact in the same manner. In this basis, the perturbing terms become off-diagonal matrix elements in the Hamiltonian and can be solved expactly to all orders. The Hamiltonian becomes

$$
H=\left[\begin{array}{cccc}
E_{z} & i k_{z} P_{z} & 0 & 0 \\
-i k_{z} P_{z} & E_{p} & \frac{\sqrt{2}}{3} \Delta & 0 \\
0 & \frac{\sqrt{2}}{3} \Delta & E_{p}-\frac{\Delta}{3} & 0 \\
0 & 0 & 0 & E_{p}+\frac{\Delta}{3}
\end{array}\right] .
$$

The energies $E_{\varepsilon}$ and $E_{p}$ refer to the conduction band and valence band energies, respectively. The quantities $P_{z}$ and $\Delta$ are defined by

$$
P_{z}=-\frac{i \hbar}{m}\left(S\left|p_{z}\right| Z\right)
$$

and

$$
\Delta=\frac{3 i \hbar}{4 m^{2} c^{2}}\left\langle X\left|\frac{\partial V}{\partial x} p_{y}-\frac{\partial V}{\partial y} p_{x}\right| Y\right\rangle
$$

Note that both $P_{z}$ and $\Delta$ are real and $\Delta$ is positive. If we now let $E^{t}=E-\frac{\hbar^{2} k^{2}}{2 m}$, then the solutions of $H u=E^{\prime} u$ give eigenvalues for $E^{\prime}$ solved by

$$
E^{\prime}=0
$$




$$
E^{\prime}\left(E^{\prime}-E_{G}\right)\left(E^{\prime}+\Delta\right)-k_{z}^{2} P_{2}^{2}\left(E^{\prime}+2 \Delta / 3\right)=0
$$

for the energies of the four states, where $E_{G}=E_{e}-E_{p}-\Delta / 3$. We label the states $\phi_{c}, \phi_{v 1}, \phi_{v 2}$, and $\phi_{v 3}$. If we expand the above solution to the first power of $k_{z}^{2} P_{z}^{2}$, we obtain the zero order solutions for the Bloch wavefunctions as

$$
\begin{aligned}
\phi_{c} & =s \downarrow \\
\phi_{v 1} & =\lfloor(x+i \gamma) 1] / \sqrt{2} \\
\phi_{v 2} & =\sqrt{1 / 3}[(x-i \gamma) \uparrow / \sqrt{2}]+\sqrt{2 / 3}[z \downarrow] \\
\phi_{v 3} & =\sqrt{2 / 3}[(x-i \gamma) \uparrow / \sqrt{2}]-\sqrt{1 / 3}[z \downarrow]
\end{aligned}
$$

and with associated energies of

$$
\begin{aligned}
& E_{c}=E_{G}+\frac{\hbar^{2} k^{2}}{2 m}+\frac{P^{2} k^{2}}{3}\left(\frac{2}{E_{G}}+\frac{1}{E_{G}+\Delta}\right) \\
& E_{v 1}=\frac{\hbar^{2} k^{2}}{2 m} \\
& E_{v 2}=\frac{\hbar^{2} k^{2}}{2 m}-\frac{2 P^{2} k^{2}}{3 E_{G}} \\
& E_{v 3}=-\Delta+\frac{\hbar^{2} k^{2}}{2 m}-\frac{P^{2} k^{2}}{3\left(E_{G}+\Delta\right)} .
\end{aligned}
$$

We notice the conduction band has a positive effective mass and the valance bands have a negative effective mass, except $\phi_{v 1}$, which is unaffected to this approximation. Coupling to other bands does make the effective mass of $\phi_{v 1}$ go negative, but not strongly so because of their weaker coupling. The $\vec{k} \cdot \vec{p}$ theory is thus seen to explain the small positive effective mass of the conduction band and the three valence bands. Two valence bands are predicted to be degenerate at $k=0$, one with a heavy negative mass and the other with a light negative mass. A split-off valence band is also predicted with a energy below the other two and a small negative effective mass.

The equation for the energy of the conduction band is quadratic in the momentum wavevector, $k$. This dependance allows us to describe the nature of the band 
near the band edge by the use of an effective mass, $m_{c}^{*}$, defined by

$$
E_{c} \equiv E_{G}+\frac{\hbar^{2} k^{2}}{2 m_{c}^{i}}
$$

We now define for future convenience the value of a dipole coupling energy, $E_{p}$, by

$$
E_{p}=\frac{2 m_{e}}{\hbar^{2}} P_{z}^{2}=\frac{2}{m_{e}}\left\langle S\left|p_{z}\right| Z\right\rangle^{2}
$$

The effective mass can now be found from equation (IV.10) as

$$
\frac{1}{m_{c}^{*}}=\frac{1}{m_{e}}\left[1+\frac{E_{p}}{3}\left(\frac{2}{E_{G}}+\frac{1}{E_{G}+\Delta}\right)\right]
$$

It can be seen that if $\Delta=0$, then $m_{e} / m_{c}^{*}=1+E_{p} / E_{G}$. The effective mass and the bandgap energy, of GaAs at $\mathrm{T}=0 \mathrm{~K}$ is found[22] to be $m_{c}^{*} / m_{\varepsilon}=0.067$ and $E_{G}=$ $1.52 \mathrm{eV}$, which from equation (IV.13) gives $E_{p}=22.54 \mathrm{eV}$. At $300 \mathrm{~K}, E_{G}=1.423$ $\mathrm{eV}$ and $m_{\mathrm{c}}^{*} / m_{e}=0.063$, giving $E_{p}=22.62 \mathrm{eV}$

We are primarily interested in the conduction band properties in the analysis of the QWEST. It is noticed that the spin-orbit coupling does not directly couple the conduction band with any other state. The spin-orbit only effects the conduction band through the mixing of valence band states, which is a higher order effect. Thus we can often approximate the above Hamiltonian in equation (IV.5) by its reduced two-by-two Hamiltonian coupling only the $S$ and $Z$ states via the $k_{z} P_{z}$ terms. Either by solving, for the eigenvalues of this reduced Hamiltonian or by solving for equation (IV.8) with $\Delta=0$, we find the quantum energies of the two states from a quadratic equation with solutions of

$$
E=\frac{E_{G}}{2} \pm \sqrt{\left(\frac{E_{G}}{2}\right)^{2}+\left(k_{z} P_{z}\right)^{2}}
$$


where the plus gives the conduction band energy and the minus gives the valence band energy. For small $\left(k_{z} P_{z}\right)^{2}$, the above equation reduces to

$$
E=\frac{E_{G}}{2} \pm\left(\frac{E_{G}}{2}+\frac{k_{2}^{2} P_{z}^{2}}{E_{G}}\right)
$$

where as before the plus gives the conduction band energy and the minus is the valence band energy. Notice the conduction band energy is the same as in equation (IV.10) with $\Delta=0$. However the valence band energy cannot be similarly obtained. The two-by-two Hamiltonian with the above energy is solved by wavefunctions which can easily be expanded to first order in $k_{z} P_{z}$ to be

$$
\begin{aligned}
& \phi_{c}=S \downarrow-i\left(k_{z} P_{z} / E_{G}\right) Z \downarrow \\
& \phi_{v}=-i\left(k_{z} P_{z} / E_{G}\right) S \downarrow+Z \downarrow
\end{aligned}
$$

with the normalization of the wavefunction not included. The value of $k_{z} P_{z} / E_{G}$ can is evaluated as follows. We first notice $k_{z}^{2} P_{z}^{2}=E_{f} E_{p}$ where $E_{f}=\hbar^{2} k^{2} / 2 m_{e}$ is the free electron energy. But the energy of an electron in the conduction band is given in the effective mass approximation by $E_{c}(k)=m_{e} / m_{c}^{*} E_{f}$ and we saw previously that $E_{p}=\left(m_{e} / m_{c}^{*}-1\right) E_{G} \simeq m_{e} / m_{c}^{*} E_{G}$. Using these observations, we find

$$
\frac{k_{z} P_{z}}{E_{G}}=\frac{\sqrt{E_{c}(k) E_{G}}}{E_{G}}=\sqrt{\frac{E_{c}(k)}{E_{G}}} .
$$




\section{B. GaAs Quantum Well Energy}

We now use the above analysis to derive formulations for the band energy of the GaAs quantum well and various related quantities such as QWEST density of states and Bloch functions. We are first concerned with the energy of the QWEST. We found previously that the density of states for the transition would be a delia function, or with zero bandwidth. But this result, as indicated in equation (III.12), is based on the energy being quadratic in the momentum vector, or the effective mass approximation. Since we are interested in finding the density of states for the transition at least to first order, we must retain the higher order terms in equation (IV.8) for the energy eigenvalues. It can also be noted that inclusion of the nonquadratic terms must be used simply to calculate the quantum well energies with any accuracy for smaller well widths where the energy of the momentum state begins to extend into the linear part of the band diagram and the effective mass approximation is no longer valid. The effective mass approximation must be adjusted for two effects. The first has been illustrated above. The solution to the quadratic equation, (IV.14), for the band energy in $k \cdot p$ theory is not quadratic in $k_{z}$ for large values of $k_{z} P_{z}$. Next, the spin-orbit coupling also affects the bands. But this latter effect is lower for the conduction bands since the spin-orbit coupling does not directly connect the conduction band to any other band in the Hamiltonian. Our approach will solve equation (IV.8) without mathematical approximation with the aid of a computer. But this does not imply our solutions will be particularly accurate. In the derivation of equation (IV.8), we neglected the coupling to the other bands by $k \cdot p$ theory. This additional coupling is given by second order perturbation theory in quantum mechanics, neglecting spin- orbit coupling, as [21]

$$
E_{i}\left(k_{z}\right)=E_{i 0}+\frac{\hbar^{2} k_{z}^{2}}{2 m_{e}}+\frac{\hbar^{2}}{m^{2}} \sum_{j \neq i} \frac{\left|k_{z}\left(\Psi_{j}\left|p_{z}\right| \Psi_{i}\right)\right|^{2}}{E_{i 0}-E_{j 0}}
$$


where we see the coupling of band " $\mathrm{i}$ " with the other bands " $\mathrm{j}$ " through the $k \cdot p$ term. This expression reduces to that of equation (IV.15) when the sum only includes two bands. This extension of the $k \cdot p$ coupling to the other bands is critical for the heavy valence band as it is seen to be totally unaffected by the coupling to the conduction band. As a measure of the accuracy of the $k \cdot p$ theory, we can calculate the value of $m^{*}$ independently for the three bands that are affected by $k \cdot p$ theory and checking for consistency. We use the relations of equation (IV.10) and room temperature values for $E_{G}$ and $E_{\mathrm{p}}$ of $1.423 \mathrm{eV}$ and $22.62 \mathrm{eV}$. This value of $E_{p}$ was derived from the conduction band effective mass and will return the original number used for this mass. We let the spin-orbit splitting be 0.341 eV|22]. From equation (IV.10) we find the effective masses of the bands to be $m_{c}^{*}=0.063, m_{v 1}^{*}=1.0, m_{v 2}^{*}=-0.104$, and $m_{v 3}^{*}=-0.305$. In comparison, the experimental values are found to be ${ }^{[22]} m_{c}{ }^{*}=$ $0.063, m_{v 1}^{*}=-0.50, m_{v 2}^{*}=-0.076$, and $m_{v 3}^{*}=-0.145$.

The valence band masses are seen to have different degrees of accuracy. The most likely cause of errors is the neglect of the coupling to all other bands as indicated in equation (IV.18). A clear measure of the effect of the other bands is seen from the calculation of $m_{v 1}^{*}$. This valence band is not affected at all by $k \cdot p$ theory in the two-band approximation, and in fact bends in the wrong direction. An examination of a GaAs band diagram[48] indicates that bands exist at energies 4.55 , $1.51,0,-0.35$, and $-12.55 \mathrm{eV}$ above and below the valence band edge, respectively. The energies at 1.51 and $\mathbf{- 0 . 3 5}$ correspond to conduction and split-off valence bands, already descriped by $k \cdot p$ theory in the two-band model. The next closest band is at $4.55 \mathrm{eV}$, about $3 \mathrm{eV}$ above the conduction band. It can be seen from equation (IV.18) that this band will reduce the inverse effective mass. The value of the reduction of $m_{e} / m_{v 1}^{*}$ from the two-band value of 1.0 to the emperical value of -2.0 is equal to -3. If we assume the other valence band inverse effective masses calculated in the two-band 
model are reduced by a similar amount, we find new values of $m^{*}$ of $m_{v 2}^{*}=-0.079$ and $m_{v 3}^{*}=-.159$, in much better agreement with theory. We measure the strength of a band coupling in equation (IV.18) by dividing by the electron free energy to obtain units of $m_{e} / m^{*}$. This unit will be seen later, in equations (IV.53) and (IV.54), to be related to the oscillator strength. In this unit the strength of the two-band coupling is about 12 for the conduction band coupling to the valence band and about 3 for coupling to the other bands. The two-band model is therefore considered to be accurate only to about $25 \%$. Nevertheless, the energy of the quantum well wavefunctions within the well region can be obtained to first order accurately using this analysis by simply matching the quadratic expansion of the solution to equation (IV.8) to the known electron effective mass in the conduction band, since this is the the only band of interest. The only energy approximation remaining is for the deviations of the energy from quadratic dependance on momentum wavevector. Our calculations indicate that for the small $\mathbf{k}$ vectors, relative to the reciprocal lattice vector, of interest here, the deviation from the effective mass approximation changes the energy of the QWEST by only about $15 \mathrm{meV}$ for the higher energy transitions. Also, deviation of the band energy from quadratic dependence on the $k$ vector only causes the density of states to increase to about $5 \mathrm{meV}$ at room temperature and much less at lower temperatures. Furthermore, even if we had perfectly accurate band energies, the quantum well energies can be off by as much as $10 \mathrm{meV}$ or more because of the great difficulties in treating the well boundary conditions. The broadening of the states by the band nonparabolicity is not the dominant broadening mechanism. Thus the approximation of using the two-band $k \cdot p$ theory seems well justified for cases of interest here.

The envelope approximation for the beh avior of the electron assumes the electron remains in a similar environment, such as the conduction band of a GaAs 
crystal. We now attempt to describe the behavior of an electron in the in homogeneous medium of a quantum well (see Fig. IV.1). We first note our quantum wells are piecewise continuous, with the well defining one region and the barriers defining another two regions. The crystals used in the experiments to be described usually contain more than one well, but we separate the wells sufficiently to prevent any coupling or interaction between wells. Since we are concerned with only three regions, the most practical approach seems to be one of describing the behavior in each of the regions as described above and then trying to define what happens at the boundary. This boundary condition determines much of the properties of the quantum well states. Yet it will be seen to be the most difficult to describe for a large number of reasons. The boundary condition for an electron at a potential barrier is normally simply taken to be continuity of the wavefunction and its derivitive normal to the boundary. This boundary condition is a direct result of first and second integrations of Schrödinger's equation across the boundary in the absence of delta function or infinite potentials. However, the boundary condition for our electron is more difficult because the envelope wavefunction is not the full wavefunction of the electron.

One of the first modifications of the boundary is to the concept of a Bloch state which remains constant from one cell to another, and the assumptions of equations (IV.1), (IV.2), and (IV.3) do not hold and need to be changed. The separation of the electron wavefunction into a product, as in equation (IV.1), is permitted in band theory because of translational invariance of the potential terms. The usual treatment of the wavefunction uses periodic boundary conditions to require the envelope take the form given in equations (IV.2) and (IV.3). This treatment is improper for real crystals. First we note that the crystal is not infinite, translational symmetry does not hold, and thus not allowing a separation of the envelope and Bloch states as simple as stated in equation (IV.1). We also note that periodic 
boundary conditions are improper and instead one should use boundary conditions which allow the wavefunction to go to zero at the edge of the crystal. If we assume equation (IV.1) to be correct momentarily, then $\Psi=0$ boundary conditions give envelopes which are sine iunctions instead of exponentials and allow $\vec{k}$ to take values half that of equation (IV.3). Negative values of $\vec{k}$ are no longer valid as they describe the same exact state which has already been described by the positive values of $\vec{k}$. Thus the number density of states per unit energy is the same as under the usual band theory. The states missing by not allowing negative values of $\vec{k}$ are made up in the density count by allowing half integer values of $2 \pi / L$ for $\vec{k}$ in addition to the normal full integer values. The improper treatment usually makes little difference for large crystals involving averages over large numbers of states, but for quantum wells in which only one state is being described, the differences are critical.

We now attempt a simple description of how the states are modified to account for the differences. We first note that for $\Psi=0$ boundary conditions, the wavefunction must be symmetric about the center of the well. This is also true for our quantum well with a finite barrier on both sides which is also symmetric about its center. A solution we might look for is one which is sinusoid and symmetric or antisymmetric about the crystal center. We first investigate, using $k \cdot p$ theory, a sinusoid envelope. The solution can be found with the simple observation that the energies resulting from the $k \cdot p$ theory are independent of the sign of $k_{z}$, as illustrated in equation (IV.8). This degeneracy of the positive and negative $k_{z}$ states allows us to renormalize the states by any linear combination of the two wavefunctions of the degenerate states. For this discussion we use the simple wavefunctions of equation (IV.16), when combined with the exponential envelope of equation (IV.2) as in equation (IV.1). We notice that equation (IV.16) is not independant of the sign of $k_{z}$ and thus the periodicity of the Bloch state wavefunction from one lattice 
cell to another breaks down as expected. The linear combinations of interest are

$$
\Psi\left(k_{z}^{n}\right)=\left[e^{i k_{z}^{n} z} \phi\left(k_{z}^{n}\right)+(-1)^{n-1} e^{-i k_{z}^{n}} \phi\left(-k_{z}^{n}\right)\right] / 2
$$

which, with the aid of equation (IV.16), becomes

$$
\left.\Psi\left(k_{z}^{n}\right)=(i)^{n-1}\left\{\cos \left(k_{z}^{n} z-\frac{\pi}{2}(n-1)\right) S^{W}+\frac{k_{z} P_{z}}{E_{G}} \sin _{1 \cdot z z}-\frac{\pi}{2}(n-1)\right) z^{W}\right\} \downarrow
$$

We have superscripted the Bloch states $S$ and $z$ because the well and barrier regions consist of similar but different materials and their Bloch states may have different wavefunctions. For an infinite well, $k_{z}^{n}=n k_{q}=n \frac{\pi}{L_{z}}$. The value of $k_{z} P_{z} / E_{G}$ was found in equation (IV.17) to be equal to the square root of the ratio of the quantum well energy to the band gap energy. This quantity takes on a maximum value of about 0.3 or less for quantum states to be discussed. We see that this form still does not go entirely to zero at the boundary of an infinite well since, for a finite $k_{z}$, a small component of the $Z$ Bloch state remains because the sine term has a maximum magnitude where the cosine term is zero. Also note that we have assumed here that the zero of the $z$ coordinate is at the center of the well. This assumption is used throughout the remainder of this chapter. In contrast, the zero of the $\mathrm{z}$ coordinate was taken at the edge of the well throughout chapter III.

A similar analysis to that above may be done for the barrier region. Only the confined states are of interest here so the electron will have a wavefunction which decays as it penetrates the barrier. We try to find such a state in GaAs by replacing $k_{z}$ with ir in equations (IV.1) through (IV.18). The envelope equation, (IV.2), becomes

$$
\Psi_{\text {env }}^{B}=e^{-\gamma z} .
$$


As before, we find that equation (IV.16) in combination with equation (IV.21) above for the envelope gives a full wavefunction of

$$
\Psi^{B}\left(\gamma^{n}\right)=e^{-\gamma z}\left[S^{B}+\left(\gamma P_{z} / E_{G}\right) Z^{B}\right] \downarrow
$$

The superscript on $\gamma$ refers to the $n$ 'th state of the quantum well. The value of $\gamma$ for this quantum state depends on the energy of the state. If we use the expression for the energy of two bands in equation (IV.15) and replace $k_{z}$ by ir we obtain

$$
E=\frac{E_{G}}{2} \pm\left(\frac{E_{G}}{2}-\frac{\gamma^{2} P_{z}^{2}}{E_{G}}\right)
$$

This is equivalent to use of the effective mass approximation with the energy below the conduction band edge being given by $\hbar^{2} \gamma^{2} / 2 m^{*}$. This barrier region is made of $\mathrm{Ga}_{1-\mathrm{x}} \mathrm{Al}_{\mathrm{X}} \mathrm{As}(0 \leq \mathrm{x} \leq 1)$. The bandgap of $\mathrm{Ga}_{1-\mathrm{x}} \mathrm{Al}_{\mathrm{X}} \mathrm{As}$ is given by $E_{G}=1.424+$ 1.247x for $x \leq 0.45$. For $0.45<x<1.0$, the bandgap is indirect and the value of the direct bandgap varies as $E_{G}=1.424+1.247 x+1.147\left(x^{2}\right.$ in this region, to a maximum of $3.018 \mathrm{eV}$ for AlAs. However the minimum gap of AlAs is indirect and has a much lower value of $2.168 \mathrm{eV}$. Since we are interested only in bound states, the effect of the electron band structure in the barrier region is not very critical and we describe the behavior of the electron in this region by an electran effective mass of ${ }^{[8]} m_{B}^{*}=0.067+0.083 x$ for a barrier with Al fraction of $x$.

The question now is how the difference in the energy gap between the GaAs and the $\mathrm{Ga}_{1-\mathrm{x}} \mathrm{Al}_{\mathbf{x}} \mathrm{As}$, or $\Delta E_{G}$, is divided between the conduction and valence bands at the interface of the two materials, a much more difficult parameter to measure. For many years it was felt that the conduction band discontinuity was $85 \%$ of $\Delta E_{G}$. However, very recent measurements ${ }^{\mid 23]}$ are consistent in pointing towards a different value of about $65 \% \Delta E_{G}$ for the conduction band discontinuity, and 
this is the value we use for the barrier height of the electron in the conduction band quantum well. Then for an Al fraction $x$ less than 0.45 we find a potential barrier height of $V_{B}=0.811 \mathrm{x} \mathrm{eV}$. For $0.45<\mathrm{x}<1.0$, the barrier potential is taken to be $V_{B}=0.811 x+0.746(x-0.45)^{2}$. This barrier height for $x>0.45$ assumes that an electron in the well region couples to the same symmetry Bloch state into the barrier region. This assumption is arbitrary and nay ueed modification in light of the future accuracy of its predictions. A possible alternative assumption allows the well region electron, at the lowest energy point with $\Gamma$ symmetry, to have a smaller barrier height by coupling to the $X$ symmetry Bloch state. This is because the bands become indirect for $x$ greater than $45 \%$ with the $X$ symmetry state becoming the lowest energy state in the conduction band and the $\Gamma$ point remaining the energy maximum for the valence band for all values of $x$. But this lower barrier height would only occur for a change in symmetry of the Bloch state across the bouudary, and is considered to be a lower probability assumption.

The quantum well eigenvalues can now be solved with the above assumptions by first assuming an energy for the state. Then from equation (IV.8) we know the value of $k_{z}$ for that energy and from equation (IV.20) the wavefunction in the well region of that energy. From the barrier height given above we can determine how far the quantum energy is below the band edge in the barrier region. Then from equation (IV.23) we determine the value of $\gamma$ for the state and from equation (IV.22) the wavefunction of the state in the barrier region. These calculations can be done for any energy and do not yet determine which energies are allowed quantum states. This quantization is performed by the boundary conditions at the boundary between the well and the barrier. Unfortunately, this boundary condition is one of the most difficult conditions to determine.

The boundary conditions for a full Hamiltonian can easily be found by a simple 
integration of the Hamiltonian across the boundary, and are given by continuity of the wavefunction, $\Psi$, and its derivative, $\partial \Psi / \partial z$, at the boundary of the two regions. $[1,2]$ However, this approach fails for the envelope approximation. The envelope cannot be assumed to have a continuous wavefunction and derivitive at the boundary. The reason is that the envelope wavefunction is not the full wavefunction of the conduction band electron. The full wavefunction is given by such expression as equations (IV.16), (IV.20), and (IV.22), where the Bloch states $S$ and $Z$ are strong functions of position of the electron. Some attempts have been made to create a new Hamiltonian based on the envelope states[24,25], with this new Hamiltonian being given simply by an effective mass approximation or better as in the $k \cdot p$ approximation. This attempt typically leads to a requirement that the envelope wavefunction, $\Psi$, and the inverse effective mass times its derivitive, $\left(1 / m^{*}\right) \partial \Psi / \partial z$, remain constant across the boundary. Unfortunately, this approach of assuming a new Hamiltonian for the envelope states, although strictly valid for the envelopes within a uniform region, has problems in being valid across a boundary because the basis states (the Bloch states, in this instance) which are used are no longer constant across the boundary. An analysis by Bastard|21] explicitly assumes that the Bloch states remain constant across the boundary and that they couple to the other $S$ and $Z$ Bloch stales across the boundary in the same relative ratio. Both of these assumptions are arbitrary and not necessarily correct. This assumption will be compared with others in relation to experimental evidence in the next chapter. We first look at what is believed to be the proper boundary conditions. We assume that that the continuity of the full wavefunction and its derivitive holds. One then has, srom equations (IV.20) and (IV.22), the following relation at the boundary $\pm L_{z} / 2$

$$
A\left[\cos \left(A_{n}\right) S^{W}+\frac{k_{z} P_{z}}{E_{G}} \sin \left(\vartheta_{n}\right) Z^{W}\right]=B e^{-\gamma^{n} L_{z} / 2}\left[S^{B}+\frac{\gamma^{n} P_{z}}{E_{G}} Z^{B}\right]
$$


from the continuity of the wavefunction across the boundary and

$$
\begin{gathered}
A k_{z}^{n}\left[-\sin \left(\theta_{n}\right) S^{W}+\frac{k_{z} P_{z}}{E_{G}} \cos \left(\theta_{n}\right) Z^{W}\right]+A\left[\cos \left(\theta_{2}\right) \frac{\partial S^{W}}{\partial z}+\frac{k_{z} P_{z}}{E_{G}} \sin \left(\theta_{n}\right) \frac{\partial z^{W}}{\partial z}\right] \\
=-B \gamma^{n} e^{\left(-\gamma^{n} L_{z} / 2\right)}\left[S^{B}+\frac{\gamma^{n} P_{z}}{E_{G}} Z^{B}\right]+B e^{\left(-\gamma^{n} L_{z} / 2\right)}\left[\frac{\partial S^{B}}{\partial z}+\frac{\gamma^{n} P_{z}}{E_{G}} \frac{\partial Z^{B}}{\partial z}\right]
\end{gathered}
$$

from the continuity of the derivitive across the boundary. The value of $\theta$ is defined by

$$
\theta_{n}=k_{z}^{n} L_{z} / 2-\frac{\pi}{2}(n-1)
$$

These boundary conditions are the proper form to achieve accurate quantum well energies. The problem is that they require knowledge of the wavefunction of the Bloch states, which has not been required up until now. However, from the symmetry properties of the Bloch states, the above can be reduced slightly. For instance, assume the boundary ocurrs at the edge of a primitive lattice cell. Then from the symmetry of the $S$ Bloch state, we know it must have a derivitive which goes to zero at the boundary. Similarly, we know that the wavefunction of the $Z$ Bloch state must go to zero at the boundary. The above equation then simplifies by a factor of two. Using these symmetry assumptiors, the above equations reduce to

$$
A \cos \left(\theta_{n}\right) S^{W}=B e^{-\gamma^{n} L_{x} / 2} S^{B}
$$

and

$$
A k_{z}^{n}\left[-\sin \left(\theta_{n}\right) S^{W}\right]+A\left[\frac{k_{z} P_{z}}{E_{G}} \sin \left(\theta_{n}\right) \frac{\partial Z^{W}}{\partial z}\right]=-B \gamma^{n} e^{\left(-\gamma^{n} L_{z} / 2\right)}\left[S^{B}\right]+B e^{\left(-\gamma^{n} L_{z} / 2\right)}\left[\frac{i \gamma^{n} P_{z}}{E_{G}} \frac{\partial Z^{B}}{\partial z}\right]
$$


It is noticed that equation (IV.27) reduces simply to continuity of the envelope wavefunction if we assume the Bloch wavefunction of the well region is similar to the Bloch wavefunction of the barrier region. However, equation (I $\sqrt{ } .28)$ is much more compli :ated and cannot be solved without detailed knowledge of the Bloch state wavefunctions. If we alter equation (IV.17) slightly, we find that $\gamma P_{z} / E_{G}$ is approximated by $\sqrt{\Delta E / E_{G}}$, where $\Delta E$ is the energy of the state below the bandgap. It is easy ' $\mathrm{s}$ see this value is about 0.4 for the GaAs bandgap of $1.43 \mathrm{eV}$ and barrier heights of about $300 \mathrm{meV}$. Assume momentarily that the derivitive of the $Z$ Bloch state is smaller than the value of the $S$ Bloch state multiplied by $k_{z}$ or $\gamma$ (this is a poor assumption since one expects the Bloch state to vary much more rapidly than the envelope). In this approximation one sees that the second terms on each side of equation (IV.27) drop out. Then the ratio of (IV.28) to (IV.27) reduces simply to

$$
k_{z}^{n} \tan \left(\theta_{n}\right)=\gamma^{n}
$$

which is the expected boundary cordition if we were to assume continuity of the envelope wavefunction and its derivitive for the $S$ Bloch state alone. Note the Bloch function drops out in this ratio automatically with no further assumption.

We are still faced with the unsolved problem of the boundary conditions for the quantum wells. Inspection of the past literature ${ }^{[3]}$ indicates that good agreement was found in the envelope approximation simply with the use of equation (IV.28). Unfortunately these energies were calculated in the past when the energy barriers were thought to be $85 \%$ of the bandgap discontinuity instead of the now known $65 \%$. A common assumption currently made in the calculation of quantum well energies $[24,25]$ is that the envelope remains continuous as before but that the derivitive of the envelope must be multiplied by the inverse effective mass to be continuous across the boundary. If we ignore the $Z$ Bloch state and only lonk at the $S$ state envelope, 
this boundary condition reduces simply to

$$
\left(\frac{k_{z}^{n}}{m_{W}^{*}}\right) \tan \left(\theta_{n}\right)=\frac{\gamma}{m_{B}^{*}}
$$

for effective masses in the well and barrier of $m_{W}^{*}$ and $m_{B}^{*}$, respectively. This boundary condition is one of the most commonly used relations in the calculation of the quantum well states. However as discussed previously, these assumptions are not necessarlly any more accurate then the ones here. Yet further problems occur because the boundary between, for instance, $\mathrm{GaAs}$ and $\mathrm{Al}_{3} \mathrm{Ga}_{7} \mathrm{As}$ is further complicated because the $\mathrm{Al}$ atoms are not uniform thoughout the boundary. Thus even if the Bloch wavefunctions were known accurately, one would need to somehow perform the proper averaging technique to get the proper boundary conditions. Ultimately we must judge the merit of which assumptions by which ones agree best with observation. Fortunately, the very simple boundery conditions of equation (IV.30) when combined with the new barrier height assumption of $65 \%$ gives nearly perfect agreement with experimental observations. Several effects will be discussed soon which have been observed to shift the quantum well energies, but these en $\sigma y$ shifts are only 2 or $3 \mathrm{meV}$ in magnitude.

The energies of the quantum wells are now calculated using the above assumptions. We calculate the transition energies for both assumptions of continuity of the envelope derivitive, with equation (N.29) above, and with this derivitive multiplied by the inverse effective mass, using equation (IV.30) above. We then compare these two results with the experimental results for best agreement. The envelope wavefunction within the well is assumed to be entirely the envelope part of equation (IV.20) for the $S$ Bloch state with the $Z$ part ignored. Similarly, we use only the envelope part of equation (IV.22) which belongs to the $S$ state. These assumptions were implicit in the form of equations (IV.29) and (IV.30). We connect the quantum 
well energy with $k_{z}$ by use of equation (IV.8) and the room temperature values|22| of $m^{*}=0.063 m_{e}, E_{G}=1.423 \mathrm{eV}$, and $\Delta=0.341 \mathrm{eV}$. We can use these values and equation (IV.13) to derive a value of $E_{p}$ equal to $22.62 \mathrm{eV}$, which is used in the solution to equation (IV.8). The energy in the barrier region is related to $\gamma$ by use of the effective mass approximation as shown in equation (IV.23). Equation (IV.23) can be made simpler by use of the effective mass definition to give the result

$$
V_{B}-E=\frac{\hbar^{2} \gamma^{2}}{2 m^{*}}
$$

where $V_{B}$ and $m_{B}{ }^{*}$ are the barrier height and the effective mass of the $\mathrm{Al}_{\mathrm{X}} \mathrm{Ga}_{1-\mathrm{X}}$ As barrier, respectively. The values of $V_{B}$ and $m_{B}{ }^{*}$ used were given previously.

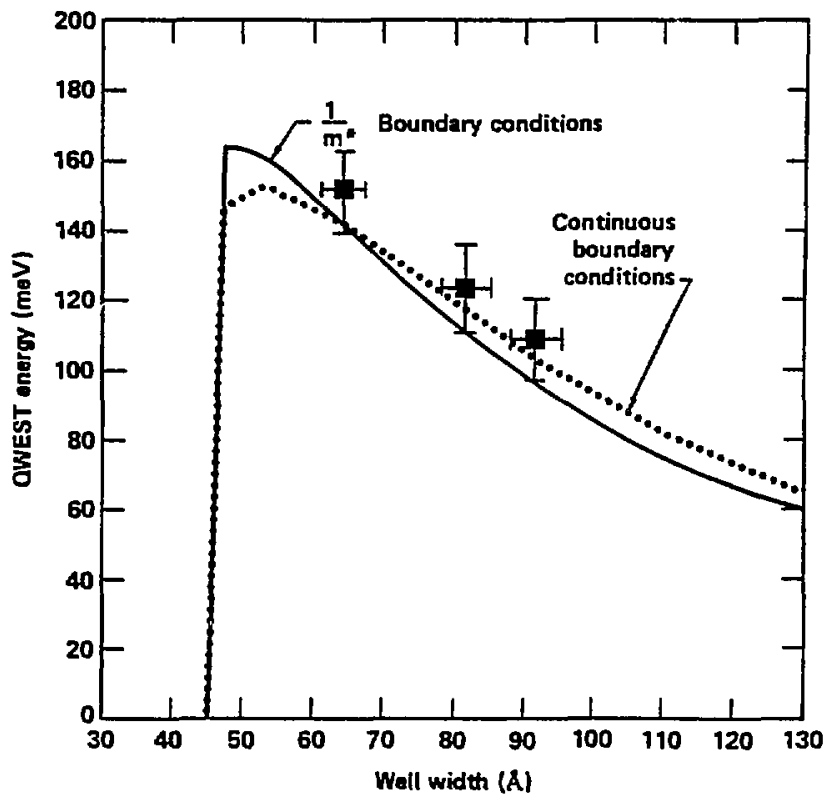

Figure IV.2: A plot of the calculated QWEST energies using the $m^{*}$ and normal boundary conditions with the experimental observations also indicated. 
A plot of the calculated QWEST energies as a function of well thickness is given in Fig. IV.2 for both assumptions of boundary conditions and a barrier of $\mathrm{Al}_{3} \mathrm{Ga}_{7} \mathrm{As}$. Also plotted is the QWEST energies for the three observed transitions. It can be seen that a much better fit to the observations is obtained with the $1 / \mathrm{m}^{*}$ boundary conditions of equation (IV,30). Thus these conditions are used for all future discussions and analysis. 


\section{GaAs QWEST Dipole and Oscillator Strength \\ GaAs QWEST Dipole}

In chapter III., we described the envelope states and the dipole between them. Quantum states in GaAs have much more structure than presented by the envelopes and in this chapter we describe how the dipole must be modified. It will be seen that the envelope approximation is very good.

The dipole between two quantum well states can be written down directly with the use of equations (IV.20) and (IV.22) for their full wavefunctions. A dipole, $\mu_{m n}$ between an upper state with envelope quantum number of $m$ and a lower state with an envelope quantum number of $n$ is given by

$$
\begin{aligned}
& \mu_{m n}=e\left(\Psi_{u}|z| \Psi_{l}\right)=\int_{-\infty}^{\infty} \Psi_{u}^{*} z \Psi_{l} d z \\
& =e \int_{-L_{z} / 2}^{L_{z} / 2}\left\{\cos \left(k_{z}^{m} z-\frac{\pi}{2}(m-1)\right) S_{u}^{W^{*}}+\frac{k_{z}^{m} P_{z}}{E_{G}} \sin \left(k_{z}^{m} z-\frac{\pi}{2}(m-1)\right) z_{u}^{W^{*}}\right\} z \\
& \quad \times\left\{\cos \left(k_{z}^{n} z-\frac{\pi}{2}(n-1)\right) S_{l}^{W}+\frac{k_{z}^{n} P_{z}}{E_{G}} \sin \left(k_{z}^{n} z-\frac{\pi}{2}(n-1)\right) z_{l}^{W}\right\} d z \\
& +2 e C \int_{L_{w} / 2}^{\infty}\left\{S_{u}^{B^{*}}+\frac{\gamma_{u} P_{z}}{E_{G}} Z_{u}^{B^{*}}\right\} z\left\{S_{l}^{B}+\frac{\gamma_{l} P_{z}}{E_{G}} Z_{l}^{B}\right\}^{-\left(\gamma_{u}+\gamma_{l}\right) z} d z
\end{aligned}
$$

The subscripts $l$ and $u$ refer the lower and upper quantum well states with envelope quantum numbers of $\mathrm{n}$ and $\mathrm{m}$, respectively. The constant $C$ is the product of the amplitude of ihe barrier wavefunctions, found as stated in the previous section by continuity of the envelope at the boundary. We have made use of the assumptions of symmetry of the quantum well about $z=0$ to simplify the integral of the barrier wavefunction. As noted before we are interested in transitions between envelope states which are of opposite parity, or equivalently, $m$ and $n$ must be of opposite parity. For example, we are most interested in the $\Psi_{1} \rightarrow \Psi_{2}$ transition. In this case the lower state has an $S$ Bloch state multiplied by a cosine function and a $Z$ Bloch state multiplied by a sine function. The upper state has an $S$ Bloch state 
multiplied by a sine function of approximately twice the lower state frequency and a $Z$ Bloch state multiplied by a cosine function of approximately twice the lower state frequency.

We now wish to integrate equation (IV.32). We first note that the Bloch states, although strong functions of position, are periodic in the lattice spacing. The envelope wavefunction, however is a slowly varying function and changes very little across a given lattice spacing. The size of the FCC lattice cell of GaAs is about $5.642 \AA$ on a side ${ }^{[22]}$ and we are interested in quantum wells with thicknesses in the range of 60 to $100 \AA$. Because the envelope varies slowly over a unit cell, we can approximate the above integral over a unit cell by the integral of the Bloch functions times the value of the envelope at the center of that unit cell. The value of the Bloch state integral over the unit cell is the same for each cell. The overall integral then reduces to a sum over all values of the envelope at the center of each cell times the overlap integral of the Bloch states. The sum over the values of the envelope can then be reduced to an integral of the envelope over the entire quantum well. For simplicity, we label the independent variables in the cosines and sines of equation (IV.32) by $\theta_{m}(z)$ and $\theta_{n}(z)$. Equation (IV.32) now reduces to

$$
\begin{aligned}
& \mu_{m n}=e\left(\Psi_{u}|z| \Psi_{l}\right)=\int_{-\infty}^{\infty} \Psi_{u}^{*} z \Psi_{l} d z \\
& =e \int_{-L_{z} / 2}^{L_{z} / 2} \cos \left(\theta_{m}(z)\right) z \cos \left(\theta_{n}(z)\right)\left\langle S_{u}^{W} \mid S_{l}^{W}\right\rangle \\
& +\left(\frac{k_{z}^{m} P_{z}}{E_{G}} \frac{k_{z}^{n} P_{z}}{E_{G}}\right) \sin \left(\theta_{m}(z)\right) z \sin \left(\theta_{n}(z)\right)\left\{z_{u}^{W}\left|z_{l}^{W}\right\rangle\right. \\
& +\frac{k_{z}^{n} P_{z}}{E_{G}} \cos \left(\theta_{m}(z)\right) \sin \left(\theta_{n}(z)\right)\left(S_{u}^{W}|z| Z_{l}^{W}\right\rangle \\
& +\frac{k_{z}^{m} P_{z}}{E_{G}} \sin \left(\theta_{m}(z)\right) \cos \left(\theta_{n}(z)\right)\left\langle z_{u}^{W}|z| S_{l}^{W}\right\rangle d z \\
& \left.+2 e C \int_{L_{z} / 2}^{\infty} z e^{-\left(\gamma_{u}+\gamma_{u}\right) z} d z\left\{\left(S_{u}^{B} \mid S_{l}^{B}\right)+\frac{\gamma_{u} P_{z}}{E_{G}} \frac{\gamma_{l} P_{z}}{E_{G}}\left\langle z_{u}^{B}\right| z_{l}^{B}\right)\right\} .
\end{aligned}
$$

We have taken the values of $(S \mid Z)$ to be zero. Note that the dipole integral has 
now reduced to four terms in the well region and one term in the barrier region. The barrier integral is simply the product of the envelope dipole part and the Bloch state overlap. The principle term in the well region is the first term, which accounts for over $\mathbf{8 0 \%}$ of the dipole. For allowed transitions of opposite parity, then one of the envelope states will change all of its cosine functions into sine functions and sine functions into cosine functions because of the $\pi / 2(m-1)$ term in $\theta_{m}(z)$. The first term in the well region integral is seen to be the dipole as calculated from the envelope wavefunctions multiplied by the overlap of the two $S$ Bloch states. The integral can be transformed into the form of equation (III.5) by letting $k_{z}^{m}=m k_{q}=$ $m \pi / L_{z}$ and changing the origin of $z$ by substitution. We then have for an infinite barrier

$$
\begin{aligned}
& e \int_{-L_{z} / 2}^{L_{z} / 2} \cos \left(\theta_{m}(z)\right) z \cos \left(\theta_{n}(z)\right) d z \\
& =(-1)^{m+n} \int_{0}^{L_{z}} \sin \left(m k_{q} z\right) z \sin \left(n k_{q} z\right) d z \\
& =(-1)^{m+n+1} L_{z} \frac{8}{\pi^{2}} \frac{m n}{\left(m^{2}-n^{2}\right)^{2}}
\end{aligned}
$$

where use has been made of equation (III.10).

The second term is more complicated since it occurs out of phase with the envelope wavefunction normally multipling the $s$ Bloch state. This term has a maximum at the well boundary. We relate this envelope integral to the envelope integral of the first term by integrating by parts. For simplicity of this calculation, we use the envelope wavefunctions of the infinite well. It will be assumed that for the finite well the two integrals will extend into the barrier by a similar amount since they are integrated over wavefunctions with identically the same $k_{z}^{n}$ values, but with a different phase. Therefore the ratio of the integrals for the infinite well should remain reasonably accurate for the finite well. Also this second term contributes less 
than $10 \%$ to the dipole strength so this approximation is quite adequate to achieve answers accurate to better than $1 \%$. We first transform the second integral to a form with the zero at the boundary edge using the infinite barrier solutions of $k_{z}^{m}=m \pi / L_{z}$ with the result

$$
\begin{aligned}
& \int_{-L_{z} / 2}^{L_{z} / 2} \sin \left(\theta_{m}(z)\right) z \sin \left(\theta_{n}(z)\right) d z \\
& =(-1)^{m+n} \int_{0}^{L_{z}} \cos \left(m k_{q} z\right) z \cos \left(n k_{q} z\right) d z \\
& =(-1)^{m+n}\left[\int_{0}^{L_{z}} \frac{n}{m} \sin \left(m k_{q} z\right) z \sin \left(n k_{q} z\right) d z-\int_{0}^{L_{z}} \frac{1}{m k_{q}} \sin \left(m k_{q} z\right) \cos \left(n k_{q} z\right) d z\right](I
\end{aligned}
$$

where we note the first term in the last equation contains the dipole integral of equation (IV.34). The second term is integrated by the use of trigonometric relations with the result

$$
\begin{aligned}
& \frac{1}{m k_{q}} \int_{0}^{L_{z}} \sin \left(m k_{q} z\right) \cos \left(n k_{q} z\right) d z \\
& \quad=\frac{1}{m k_{q}} \int_{0}^{L_{z}} \frac{1}{2}\left(\sin \left((m+n) k_{q} z\right)+\sin \left((m-n) k_{q} z\right)\right) d z \\
& =\frac{1}{m k_{q}^{2}} \frac{1}{2}\left(\frac{2}{m+n}+\frac{2}{m-n}\right) \\
& =\frac{4 L_{z}}{\pi^{2}} \frac{1}{m^{2}-n^{2}}
\end{aligned}
$$

where the parity properties of the allowed $m$ and $n$ values are used and note the normalizing constant $2 / L_{z}$ has been multiplied in the last step. We now can find the value of the integral of equation (IV.35) as

$$
\begin{aligned}
& \int_{-L_{z} / 2}^{L_{z} / 2} \sin \left(\theta_{m}(z)\right) z \sin \left(\theta_{n}(z)\right) d z \\
& =(-1)^{m+n}\left[\frac{-8 L_{z}}{\pi^{2}} \frac{n^{2}}{\left(m^{2}-n^{2}\right)^{2}}-\frac{4 L_{z}}{\pi^{2}} \frac{1}{m^{2}-n^{2}}\right] \\
& =(-1)^{m+n}\left(\frac{-4 L_{z}}{\pi^{2}}\right) \frac{m^{2}+n^{2}}{\left(m^{2}-n^{2}\right)^{2}} .
\end{aligned}
$$


This integral is now ratioed with the integral of equation (IV.34) to find the ratio of the integrals, $R$, is given by

$$
R=\frac{1}{2} \frac{m^{2}+n^{2}}{m n}
$$

Although derived for an infinite barrier, this ratio is assumed to be valid for any barrier height as discussed above.

The two remaining terms are treated the same as the second term. We calculate the ratio of this term to the first term for the infinite well and assume the ratio remains constant for any barrier height. As before, we begin by converting the integral to a form with the zero at the edge of the boundary. This gives

$$
\begin{aligned}
& \int_{-L_{z} / 2}^{L_{z} / 2} \cos \left(O_{m}(z)\right) \sin \left(\theta_{n}(z)\right) d z \\
& =(-1)^{m+n+1} \int_{0}^{L_{z}} \sin \left(m k_{q} z\right) \cos \left(n k_{q} z\right) d z \\
& =(-1)^{m+n+1} \frac{4}{\pi} \frac{m}{m^{2}-n^{2}} .
\end{aligned}
$$

A similar term for the last integral in the well region can be solved by a simple exchange of the variables $m$ and $n$. These last two terms take their dipoles between the Bloch states instead of between the envelopes. The magnitude of this dipole can be determined from the earlier $k \cdot p$ theory. We do not know the sign of this dipole but we can nevertheless determine the sign of this term, important because this term can subtract or add to the other terms, by relation to the value of $P_{z}$. Using the relations (IV.6) and the commutation relations of (III.6) and (III.7) with the free electron mass instead of the effective mass, we obtain

$$
P_{z}=E_{G}\langle S|z| Z\rangle
$$


With the aid of equations (IV.39) and (IV.40) the third term of equation (IV.33) becomes

$$
\begin{aligned}
& \left.\int_{-L_{z} /}^{L_{z} / 2} \frac{k_{z}^{n} P_{z}}{E_{G}} \cos \left(\theta_{m}(z)\right) \sin \left(\theta_{n}(z)\right)\left\langle S_{u}^{W}|z| Z\right\}^{W}\right\rangle d z \\
& \quad=\frac{k_{z}^{n} P_{z}^{2}}{E_{G}^{2}}(-1)^{m+n+1} \frac{4}{\pi} \frac{m}{m^{2}-n^{2}} \\
& \quad=\frac{k_{q} P_{z}^{2}}{E_{G}^{2}}(-1)^{m+n+1} \frac{4}{\pi} \frac{m n}{m^{2}-n^{2}} .
\end{aligned}
$$

We have used $k_{z}^{n}=n k_{q}$. The fourth term is identical to the above term with the exchange of variables $n$ and $m$. It is then seen that the fourth term is identical in all respects to the third term except that the sign is opposite. Thus these two terms cancel one another and drop from the integral for the dipole.

The remaining terms contain an overlap integral, such as $\left\langle S_{u} \mid S_{l}\right\rangle$ or $\left\langle Z_{u} \mid Z_{l}\right\rangle$. The $S$ and $z$ Bloch states are based on the atomic potential, as discussed in the section on $k \cdot p$ theory, and are the same for all $k$ vectors. The overlap integral is not necessarily unity, however, because the reference frames of the two states can be rotated from one another. The rotational properties of these states are given by E. O. Kane[20]. The $\mathrm{S}$ Bloch state is rotationaly symmetric and indeed has a unity overlap. The $\mathrm{Z}$ Bloch state rotates as the crystal axis, giving their overlap equal to the cosine of the angle between the lower and upper quantum state $k$ vector, or

$$
\left\langle Z_{u} \mid Z_{l}\right\rangle=\frac{k_{z}^{m} k_{z}^{n}+k_{t}^{m} k_{t}^{n}}{\sqrt{k_{z}^{m 2}+k_{t}^{m 2}} \sqrt{k_{z}^{n 2}+k_{t}^{n 2}}} .
$$

For low denstities and temperatures, the value of the overlap is close to unity as both the upper and lower states are have a very small transverse $k_{t}$ component and both $k$ vectors point normal to the boundary surface. As estimate of the above overlap can be found from effective mass theory, where we take the value of $k_{z}$ 
to be proportional to the square root of the quantum energy of the state and the value of $k_{t}$ to be a distribution from zero to the square root of the thermal energy, with the same proportionality constant. The minimum overlap integral can now be estimated by

$$
\left\langle z_{u} \mid z_{l}\right\rangle=\frac{\sqrt{E_{u} E_{l}}+E_{t h}}{\sqrt{E_{l}+E_{t h}} \sqrt{E_{u}+E_{t h}}}
$$

where $E_{l}, E_{l}$, and $E_{t h}$ are the lower quantum state energy, the upper quantum state energy, and the thermal energy, respectively. Taking the values of $E_{l}, E_{l}$, and $E_{t h}$ to be 50,180 , and $25 \mathrm{meV}$ respectively, the minimum velue of $\left\langle Z_{u} \mid Z_{l}\right\rangle$ becomes 0.867 . For a given transition, we must evaluate this term by using a thermally weighted average over all possible transverse $k$ vectors for a given temperature.

The second term is also multiplied by a term of $k_{z}^{m} k_{z}^{n} P_{z}^{2} / E_{G}^{2}$. This term can be seen with the aid of equation (IV.17) to reduce to $\sqrt{E_{l} E_{u}} / E_{G}$. This term will take on the value of 0.066 using as values of $E_{l}$ and $E_{u}$ and $E_{G}$ the energies $0.05,0.180$, and $1.43 \mathrm{eV}$, respectively. We saw earlier from equation (IV.38) that the envelope dipole of this second term was about $5 / 4$ times the value of the first terms envelope dipole for a $1 \rightarrow 2$ transition. Thus this second term contributes less than $10 \%$ to the dipole. Because of the small contribution of this term, we can approximate the Bloch state overlap factor of equation (IV.43) by unity with good accuracy. This approximation is consistent with the other approximations in terms of accuracy and eliminates the difficulty of having a dipole that has a dependance on density and temperature.

The approximations for the overlap of the Bloch states also apply to the barrier region with similar results. However, equation (IV.17) must be modified with the aid of equation (IV.23) to give 


$$
\frac{\gamma_{u} \gamma_{l} P_{z}^{2}}{E_{G}^{2}}=\frac{\sqrt{V_{B}-E_{u}} \sqrt{V_{B}-E_{l}}}{E_{G}}
$$

where $V_{B}$ is the barrier height given earlier.

The dipole integral of equation (IV.33) now reduces to a much simpler form given by

$$
\begin{aligned}
& \mu_{m n}=e\left(\Psi_{u}|z| \Psi_{l}\right)=\int_{-\infty}^{\infty} \Psi_{u}^{*} z \Psi_{1} d z \\
& =e \int_{-L_{z} / 2}^{L_{z} / 2} \cos \left(k_{z}^{m} z-\frac{\pi}{2}(m-1)\right) z \cos \left(k_{z}^{n} z-\frac{\pi}{2}(n-1)\right) d z \times \frac{1+\frac{m^{2}+n^{2}}{2 m n} \sqrt{E_{u} E_{l}} / E_{G}}{\sqrt{1+E_{u} / E_{G}} \sqrt{1+E_{l} / E_{G}}} \\
& +2 e C \int_{L_{z} / 2}^{\infty} z e^{-\left(\gamma_{u}+\gamma_{l}\right) z} d z \times \frac{1+\sqrt{V_{B}-E_{u}} \sqrt{V_{B}-E_{l} / E_{G}}}{\sqrt{1+\left(V_{B}-E_{u}\right) / E_{G}} \sqrt{1+\left(V_{B}-E_{l}\right) / E_{G}}}
\end{aligned}
$$

where we have included the Bloch state normalization factor in the denominator. For convenience, we will define the quantities $R_{W}$ and $R_{B}$ by

$$
R_{W} \equiv \frac{1+\frac{m^{2}+n^{2}}{2 m n} \sqrt{E_{u} E_{l}} / E_{G}}{\sqrt{1+E_{u} / E_{G}} \sqrt{1+E_{l} / E_{G}}}
$$

and

$$
R_{B} \equiv \frac{1+\sqrt{V_{B}-E_{u}} \sqrt{V_{B}-E_{l} / E_{G}}}{\sqrt{1+\left(V_{B}-E_{u}\right) / E_{G}} \sqrt{1+\left(V_{B}-E_{l}\right) / E_{G}}} .
$$

Using these definitions the dipole can be written in a yet simpler form of

$$
\begin{aligned}
& \mu_{m n}=e\left(\Psi_{u}|z| \Psi_{1}\right)=\int_{-\infty}^{\infty} \Psi_{u}^{*} z \Psi_{1} d z \\
& =e \int_{-L_{z} / 2}^{L_{z} / 2} \cos \left(k_{z}^{m} z-\frac{\pi}{2}(m-1)\right) z \cos \left(k_{z}^{n} z-\frac{\pi}{2}(n-1)\right) d z \times R_{W} \\
& +2 e C \int_{L_{z} / 2}^{\infty} z e^{-\left(\gamma_{z}+\gamma_{1}\right) z} d z \times R_{B}
\end{aligned}
$$


The dipole integral is now very similar to the integral expected simply from the envelope of the $\mathrm{S}$ Bloch state solved for the finite barrier, but with some reduction given by the factors $R_{W}$ and $R_{B}$. As an example, using values for $E_{u}, E_{l}, E_{G}$, and $V_{E}$ of $.180, .050,1.43$ and $.243 \mathrm{eV}$, respectively, we find that $R_{W}$ and $R_{B}$ equal 1.003 and .9895 , respectively, or 1.00 to the degree of approximation valid here.

\section{GaAs QWEST Oscillator Strength}

In chapter III. we defined an oscillator strength, $f$, of an optical dipole transition between two quantum states by equation (IIl.14), which we repeat here as

$$
f=\frac{2 m_{e} \omega}{\hbar}(z)^{2}
$$

where $m_{e}$ is the free electron mass, $w$ is equal to the energy difference between the states divided by $\hbar$, and $e(z)$ is the dipole between the states $s$ defined in equation (IV.32) for the wavefunctions under consideration here. This form of the oscillator strength is fundamentally derived using the quantum mecharical formula for the dipole interaction of two quantum states with an electric field $\left.\right|^{\mid 15\}}$ and comparing with the definition based on the classical response ${ }^{[10]}$ of a bound electron.

The oscillator strength of a GaAs QWEST is ca' ulated using the energies of the states as solved by the method of section $B$ and with the dipole solved as indicated in equation (IV.47). The factors $R_{W}$ and $R_{B}$ are taken to be unity with $1 \%$ accuracy. The oscillator strengths of a well with $\mathrm{Al}_{3} \mathrm{Ga}_{7} \mathrm{As}$ barriers and GaAs well thicknesses of 65,82 , and $92 \AA$ are found to be $12.1,13.2$, and 13.7 , respectively. In contrast to the quantum well energies and dipole, the oscillator strength is a slow function of well width. In chapter III, the oscillator strength for an infinite well was found in equation (III.16) to be $0.96 \mathrm{~m}_{e} / \mathrm{m}^{*}$ or about 15.2 at room temperature, and independent of well width. Quantum wells with finite barriers will have smaller oscillator strengths because the envelope wavefunctions of the two states involved 
in the transition will extend into the barriers different distances, lowering dipole integral. Some insensitivity of the oscillator strength to the well width nevertheless remains because as the energies of the quantum well states are lowered substantially by extension of the envelope wavefunctions into the barrier, the dipole is increased similarly to compensate in the expression for the oscillator strength.

The oscillator strength also obeys a sum rule[18] known as the Thomas-ReicheKuhn sum rule. This sum rule is derived in the quantum mechanics text|18] by $\mathrm{E}$. Merzbacher, among others, and for a single electron transition is given by

$$
\sum_{k} f_{k \varepsilon}=1
$$

based on the definition of the oscillator strength, $f_{k s}$, similar to that above and given by

$$
f_{k s} \equiv \frac{2 m_{e} w_{k e}}{\hbar}\left(z_{k e}\right)^{2}
$$

Note this nscillator strength need not necessarially be equal to the above definition since this quantity is simply an element occuring in a sum oyer states afler some manipulation. However it happens to be identisal. The sum in equation (IV.49) must be over all states, both bound and unbound. The quantities $\omega_{k s}$ and $\left(z_{k 8}\right\rangle^{2}$ are defined by

$$
\omega_{k s} \equiv\left(\vec{E}_{k}-E_{\theta}\right) / \hbar
$$

and

$$
\left\langle z_{k s}\right\rangle^{2} \equiv\left|\int \Psi_{k}^{*} z \Psi_{\theta} d z\right|^{2}
$$


The oscillator strength is positive when coupling to energies above $E_{0}$ and negative when coupling to lower energies. We observe that the derivation of this sum rule is sufficiently fundamental and general so as to also apply to our system. The derivation relys only on the comutation relations $\left[z, p_{z}\right]=i \hbar$ and $[H, z]=-\frac{i \hbar}{m} p_{z}$ which apply to all quantum mechanical systems. The sum rule includes both positive and negative values and includes all dipole couplings, even to nonbound states. Thus the sum rule is useful for giving limits on the strength of a given oscillator strength only for transitions from the lowest energy state, where all terms in the sum rule are positive. The QWEST does not occur between a ground state. The sum rule still applies, but does not directly tell us anything about the QWEST oscillator strength from fundamental principles.

Some properties of the oscillator strength of the QWEST can be developed, however. To see in detail how the oscillator strength applies to GaAs quantum wells and their transitions, we first look at the results of Ridley[21]. Ridley's expression for the energy bands of GaAs was given by $k \cdot p$ theory in equation (IV.18). This equation is reduced to give the effective mass, $m^{*}$, of the electron as

$$
\frac{m_{e}}{m_{i}^{*}}=1+\frac{2}{m_{e}} \sum_{j \neq i} \frac{\left|k_{z}\left\langle\Psi_{j}\left|p_{z}\right| \Psi_{i}\right\rangle\right|^{2}}{E_{i 0}-E_{j 0}}
$$

where $E_{i 0}$ are the $\mathrm{k}=0 \mathrm{Bloch}$ ilate energies and the dipoles are between the Bloch states. We now note with the use of equation (III.7), the oscillator strength can be written as

$$
\begin{aligned}
f_{k s} & \equiv \frac{2 m_{e}\left(E_{k}-E_{\theta}\right)}{\hbar^{2}}\left(z_{k_{\theta}}\right)^{2} \\
& =\frac{2}{m_{e}} \frac{\left|\left\langle\psi_{k}\left|p_{z}\right| \psi_{s}\right\rangle\right|^{2}}{E_{k}-E_{\theta}}
\end{aligned}
$$

Comparing equations (IV.53) and (IV.54), we see that 


$$
\frac{n_{e}}{m_{i}^{*}}=1-\sum_{j \neq i} f_{j i}
$$

where the sum is over all Bloch states. From the conduction band oscillator strength of $\mathbf{0 . 0 6 7}$, we find that the oscillator sum is equal to $1-m_{e} / m^{*}$ or about -14 . Whereas the conduction band summation of interband oscillator strengths is large and negative, $t$, \& valence band has a large positive sum.

It might appear that the Thomas-Reiche-Kuhn sum rule either implies all effective masses nust be infinite for all bands or else is invalid. However the sum in equation has not included all possible dipole transitions. The sum was over interband transitions which have their dipole between the Bloch states and the envelope states remain nearly constant. But we must sum over all transitions for the sum rule to apply. Thus we must include ali possible intraband dipole transitions between the envelope states (such as the QWEST) between each state and its neighbor in the band. This sum can be found either by summing equation (III.16) (which is independant of well width even up to large crystals) or noting that an artificial Hamiltonian can be created for the conduction band electron with a smaller effective mass which will yield a sum rule equal to unity in terms of its effective mass which will be equal to $\mathrm{m} / \mathrm{m}^{*}$ when normalized to the free electron mass. Similarly, the valence band will have a large negative sum of oscillator strengths over its intraband envelupe state transitions. Thus we see the sum of equation (IV.55) plus the envelope state transition oscillator strength 3 does indeed sum to unity and the Thomas-Reich-Kuhn sum rule continues to apply.

The k.P theory shows us that the coupling between the envelope states and Bloch states causes the sum of oscillator strengths to separate into two large groups of opposite sign, with the interband conduction band transitions between Bloch states having a large negative sum of $1-\mathrm{m} / \mathrm{m}^{*}$ and the intraband conduction band 
transitions between envelope states having a large positive sum of $\mathrm{m} / \mathrm{m}^{*}$. The reciprocity of the two systems, the envelope states and the Bloch states is deep and each system gains greatly from the other by the their coupling from the $k \cdot p$ perturbation. For instance, the energy of the Bloch states is easy to understand from the nature of the atomic energy states, but it is difficult to understand why these atomic Bloch states would have a dipole between them of nearly $7 \mathrm{e}-\AA$. (The size of a primitive cell is about $2 \AA$ and the dipole size is usually a small fraction of the physical size, but the GaAs atomic dipole is bigger than the atoms!). On the other hand the dipole of envelope state transitions is readily understandable from the size of the wavefunctions, but it is hard to understand why the energy should be so high. Obviousity the $k \cdot p$ perturbation has allowed a highly favorable trade to take place between the two systems and the measure of that trade is given by the oscillator strength splitting and therefore the inverse effective mass. The intricacies of the interaction are very interesting ${ }^{[20,21]}$, but beyond the scope of this thesis. 


\section{Energy Broadening Mechanisms}

In chapter III. the optical absorption of the QWEST was shown to have zero bandwidth. In this section we attempt to determine the mechanisms which lead to a finite absorption bandwidth.

Several mechanisms are important. First the density of states for the QWEST was seen in chapter III. to be infinitely narrow only for parabolic bands and conservation of the transverse momentum during a transition in an infinite well. Real bands and transitions depart from this ideal. Second, the transition has a finite lifetime which leads to some linewidth. Third, the energy of the transition depends on the well width. If some variations occur in the width of the well from one location to another, then the QWEST energy is inhomogeneously broadened by this effect. An attempt is made to determine the magnitude of each of these effects and offer an explaination of the observed linewidths. All of these broadening effects contain large uncertainties at this time, thus no definitive conclusions can truly be made here, instead only a qualitative indication.

\section{Density of QWEST states}

Two effects give a finite bandwidth to the density of states. One is deviation of the energy bands from parabolic dependence on the wavevector. The second is the dependence of the effective electron barrier height on transverse momentum wavevector. These two effects are very nearly equal for quantum wells with our parameters.

The calculation begins by solving for the quantum well energies as a function of the transverse $\mathrm{k}$ vector. We continue to use the material poperties as described in sections A. and B., and the boundary condition (IV.30) does not change. However, the equations relating the energy and the propogation vectors in the two regions are changed to include the transverse energy. The energy in the well region is described 
in equation (IV.8) and now becomes

$$
E^{\prime}\left(E^{\prime}-E_{G}\right)\left(E^{\prime}+\Delta\right)-\left(k_{z}^{2}+k_{t}^{2}\right) P_{z}^{2}\left(E^{\prime}+2 \Delta / 3\right)=0
$$

where $k_{t}$ is the transverse wavevector. Similarly, we modify equation (IV.31) for the barrier region to give

$$
V_{B}-E=\frac{\hbar^{2} \gamma^{2}}{2 m_{B}^{*}}-\frac{\hbar^{2} k_{f}^{2}}{2 m_{B}^{*}}
$$

From continuity of the wavefunction across the boundary, the transverse wavevector is taken to be the same in both the barrier and well region. The additional kinetic energy from this transverse momentum is not equal in the two regions, however. This energy difference can be described with parabolic bands in the effective mass approximation. The transverse kinetic energy of the well region is $\hbar^{2} k_{t}^{2} / 2 m_{W}^{*}$ but in the barrier region is $\hbar^{2} k_{t}^{2} / m_{B}^{*}$. The effective mass in the well region at room temperature is $0.063 m_{e}$ and that of the $\mathrm{Al}_{.3} \mathrm{Ga}_{.7} \mathrm{As}$ barrier is approximately $0.088 m_{e}$. Thus an electron at room temperature with a transverse kinetic energy in the lower quantum state of $25 \mathrm{meV}$ has a transverse kinetic energy in the barrier region about $30 \%$ lower. This energy difference is equivalent to a reduction of the barrier height, in the one dimensional calculation, by the same energy difference or about $7 \mathrm{meV}$. This effective lowering of the barrier height depends on the magnitude of the transverse momentum and thus gives some bandwidth to the density of states. The finite bandwidth of the QWEST thus arises from both the nonparabolic part of equation (IV.56) and the change in effective barrier height. 

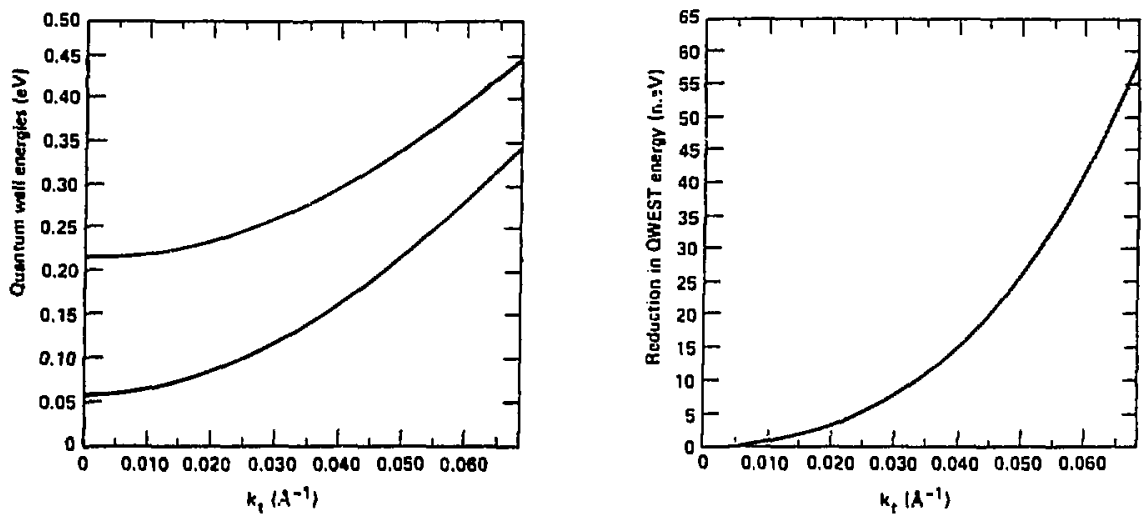

Figure IV.3: a.) A plot of the quantum well energies versus transverse $k$ vector for the two lowest energy quantum well states. b.) A plot of the the magnitude of QWEST energy reduction from the $k_{t}=0$ value as a function of $k_{t}$.

The solution of the transverse band energies including both effects is given in Fig. IV.3a for a $65 \AA$ thick well of $\mathrm{GaAs}$ with a barrier of $\mathrm{Al}_{3.3} \mathrm{Ga} .7 \mathrm{As}$. In Fig. IV.3b the reduction in the QWEST energy as the transverse wavevector increases is plotted. It can be seen that as the thermal energy of the electrons in the lower state increases towards $25 \mathrm{meV}$ above the $k_{t}=0$ edge, the transverse wavevector takes on values of about $0.022 \AA^{-1}$. At this transverse wavevector, this reduction in QWEST energy is about $4 \mathrm{meV}$. The relative importance of the reduction of the effective barrier height is found by letting the bands be perfectly parabolic in a similar calculation. The reduction in QWEST energy with transverse momentum is very similar in form with very close to half the QWEST energy reduction found with both effects included. The two effects are thus very nearly equal for quantum wells with the parameters we use.

The density of states for the QWEST is now derived in terms of the band energies of the lower and upper quantum states, $o_{1} E_{l}\left(k_{t}\right)$ and $E_{u}\left(k_{t}\right)$, respectively. 
The QWEST energy, $E_{Q}\left(k_{t}\right)$, is defined in terms of quantum well energies by

$$
E_{Q}\left(k_{t}\right)=E_{u}\left(k_{t}\right)-E_{l}\left(k_{t}\right)
$$

We first calculate the density of states for the lower quantum well state assuming the bands are spherically symmetric in the direction of the $k$ vector. If we rederive the density of states equation (II.11) without assuming $E_{l}\left(k_{t}\right)$ to be quadratic as we did in equations (II.8) and (II.10), then the density of states can be written

$$
\rho_{l}\left(E_{l}\right) d E_{l}=\frac{0}{2 \pi} \frac{k_{t}}{d E_{l}\left(k_{l}\right) / d k_{t}} d E_{l}
$$

This equation is solved numerically to give a solution very similar to that illustrated in Fig. II.1, with the difference rom the nonparabolic nature of the bands only barely observable.

The QWEST energy density of states depends on the population distribution in the lower state. The spread of electrons among the transverse momentum states is dependent on both doping density and temperature. Furthermore, both broadening effects are very dependent on the thickness of the quantum well region. The probability of any of the lower states being occupied is given by the Fermi factor, $F\left(E_{l}\right)$, which is given by

$$
F(E)=\frac{1}{1+e^{(E-\mu) / k T}}
$$

where is the Fermi energy of the electron. The Fermi level determines the electron density from the integral over all states to the probability of an electron being in that state. If we label the surface density as $\rho_{B}$, then the surface density of electrons per well is given by 


$$
\rho_{e}=\int \rho\left(E_{l}\right) F\left(E_{l}\right) d E_{l} .
$$

Only the lower quantum state is included in the above integral over transverse states. The upper quantum states are assumed to be sufficiently high in energy so as to not be populated. This assumption is valid because the QWEST energies are over $100 \mathrm{meV}$ but the experimental temperatures are $25 \mathrm{meV}$ and below.

The density of QWEST states is now calculated for a given Fermi level and temperature. The density of QWEST states is expected to have the narrowest linewidth for low Fermi energies and low temperatures, since the electrons would then be concentrated in the low energy parabolic region of the lower state where we would expect zero bandwidth. However if either the doping is high, requiring the Fermi level at zero temperature to move up into the nonparabolic region of the band, or the temperature is high, allowing the electron the freedom to move into the upper part of the transverse band, the QWEST density of states will broaden. This QWEST density of states, $\rho_{Q}\left(E_{Q}\right)$, is found from the equation

$$
\rho_{Q}\left(E_{Q}\right) d E_{Q}=\rho_{l}\left(E_{l}\right) F\left(E_{l}\right) \frac{d E_{l}}{d E_{Q}} d E_{Q} .
$$

We calcuate the QWEST density of states for some of the conditions under which experiments are performed. The calculation is performed for several different Fermi levels. The Ferm' 'evel also determines the surface density of states, and the Fermi level chosen is that which corresponds best with the actual surface density of the well. All the wells used in our experiments were doped to have a surface density of $4 \times 10^{11} / \mathrm{cm}^{2}$. The actual doping varied somewhat as will be discussed in the next chapter. The density of states at room temperature varied very little with the surface charge density, and the low temperature density of states varied slowly but was almost negligable in comparison with the other broadening mechanisins. 
Therefore small differences in surface charge density do not change the density of states significantly. Illustrated in Fig. IV.4a is the calculated QWEST density of states for the $65 \AA$ thick well with an electron thermal energy of $3 \mathrm{meV}$ or a temperature of about $35 \mathrm{~K}$. The Fermi energy for this calculation was at $70 \mathrm{meV}$, or $15 \mathrm{meV}$ above the $55 \mathrm{meV}$ energy of the $k_{t}=0$ band edge of the lower state (all energies are measured relative to the band edge in bulk GaAs). The surface density, $\rho_{8}$ at this Fermi level is $4.32 \times 10^{11} / \mathrm{cm}^{2}$. The FWHM of the distribution of energies is seen to be about $2.4 \mathrm{meV}$ for this temperature. As the temperature is increased to room temperature with $25 \mathrm{meV}$ thermal energies, the density of states broadens as illustrated in Fig. IV.4b. The Fermi level at this temperature decreases to about $45 \mathrm{meV}$ or $10 \mathrm{meV}$ below the lowest energy quantum state, for a surface density of $3.76 \times 10^{11} / \mathrm{cm}^{2}$. It can be seen that the FWHM has now increased to about $7.4 \mathrm{meV}$ at room temperature.

The QWEST density of states linewidth is also a function of the well thickness. A larger thickness well has a smaller wavevector in the $\mathrm{z}$ direction and does not place the electron as far into the nonparabolic region as does the thinner well. Thus a larger thickness weil has a much reduced linewidth in its density of QWEST states. We illustrate the QWEST density of states for an $82 \AA$ thick well in Fig. IV.4c. This calculation is at room temperature (25 meV) with a Fermi energy of $31 \mathrm{meV}$, which is $10 \mathrm{meV}$ below the lowest energy quantum state at $41 \mathrm{meV}$. This Fermi level corresponds to a surface electron density of $3.68 \times 10^{11} / \mathrm{cm}^{2}$. This density of states can be seen to have a FWHM of $6.3 \mathrm{meV}$. This linewidth is $0.9 \mathrm{meV}$ below that of the $65 \AA$ thick well at room temperature. 

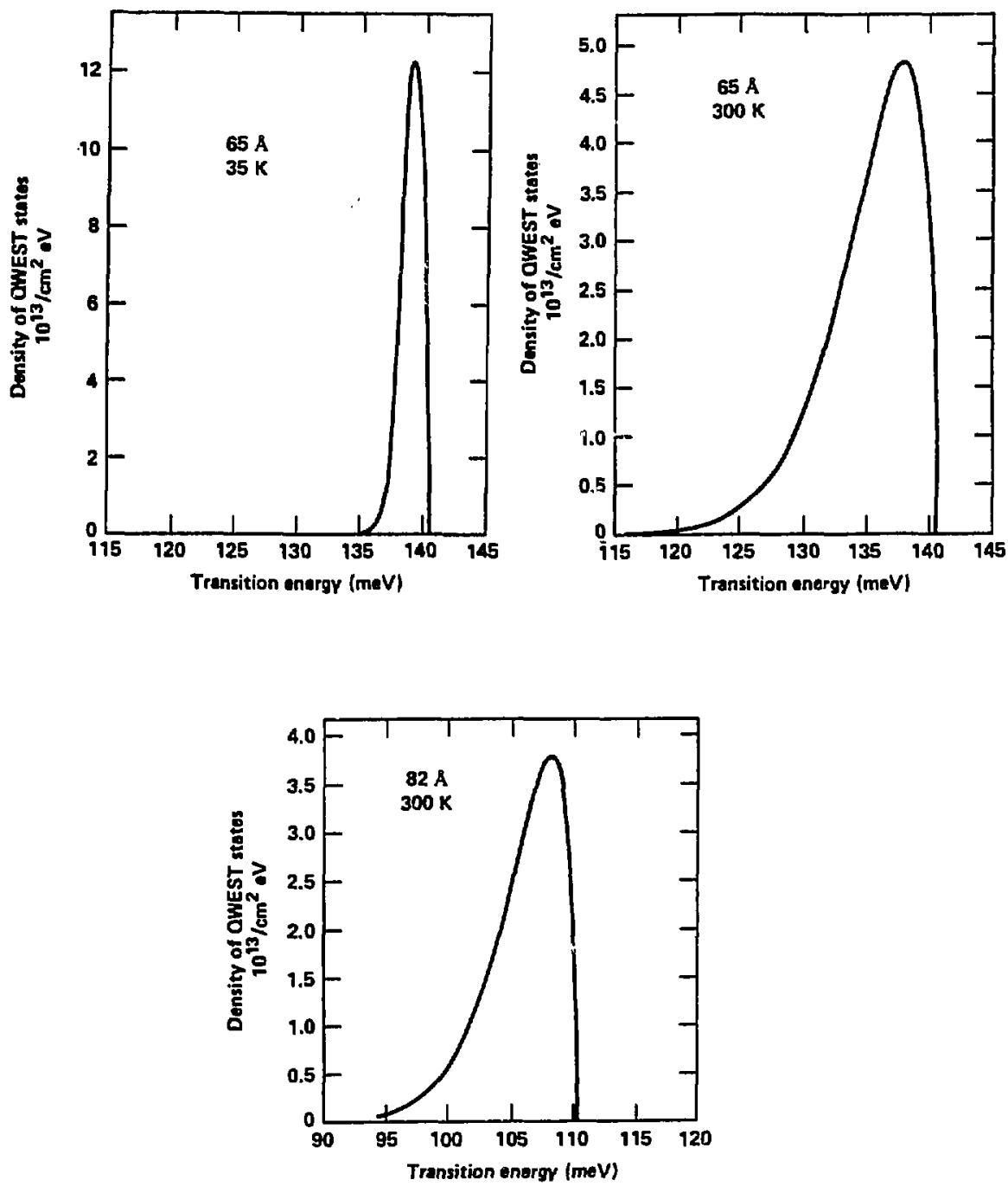

Figure IV.4: a.) A plot of QWEST density of states for 65 A well at $35 \mathrm{~K}$. b.) QWEST density of states for a $65 \mathrm{~A}$ well at $300 \mathrm{~K}$. c.) QWEST density of states for an $82 \mathrm{~A}$ well at $300 \mathrm{~K}$. 
It must be noted that some serious uncertainties as to the validity of this calculation exists. Most all of the uncertajntity concerns the degree to which real GaAs bands are nonparabolic. Of the many parameters used to calculate the properties of the QWEST, this parameter is one of the few which has no emperical verification. This term instead was derived based on the $k \cdot p$ theory between two bands and is subject to the limitations both of the theory and limited number of bands used. In the begining of seation B., the accuracy of $k \cdot p$ theory was shown to be imperfect at prodicting the effective masses of both conduction and valence bands simultaneously, with indications that the imperfections arose from the limited number of bands incorporated into the theory. The coupling to other bands was shown to have as much as a $30 \%$ effect. The theory works well at predicting energies because the major part of this energy term can be found emperically from the effective mass. Whereas the error in the energy terms might only be $10 \%$, the error in the bandwidth of the QWEST density of states is only accurate to $30 \%$. 


\section{Lifetime Broadening}

The upper state can relax back to the lower state elastically simply by rotating its momentum vector so as to convert some of its momentum perpendicular to the barrier to momentum transverse to this direction (see Fig. IV.5). This effect allows the electron to return to the lower state via an inelastic collision and thus gives the relaxation a very high speed. For sufficiently high excitation of this electron gas, the inelastic scattering among the electrons heats the electron gas relative to the latlice temperature. The QWEST absorption and refractive index would thereby lower since the QWEST energy would then be a smaller multiple of the temperature times Boltzmann's constant, and the electron gas would quickly reach equilibrium ( in a time suspected to be much less than a picosecond). The relaxation of the electron gas temperature to the lattice temperature is much slower and has been measured to be several picospconds, $\left.\right|^{[27]}$
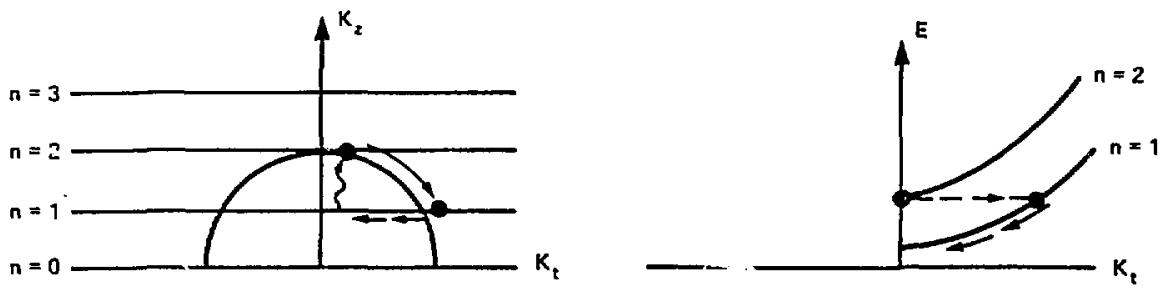

Figure IV.5: A diagram of the electron path during its relaxation from the upper quantum well state. The initial process is an elastic scattering to the lower band, followed by a slower inelastic decay to the lattice temperature. The plot on the left is of the allowed wavevectors of a quantum well. The transverse wavevectors are suffiniently close to be drawn as a line. Note the lowest energy quantum well is the $n=1$ state. A constant energy surface is also indicated. The plot on the right illustrates the energy of the two lowest quantum well states as a function of transverse wavevector. 
The process by which an electron looses momentum is similar to the erectron processes which determine the mobility of an electron in the conduction band of GaAs. An estimate of the speed of this process might be deduced from the measured mobility of the quantum well. The mobility, $\mu_{e}$, is related to the scattering time, $\tau$ of an electron by the expression[20]

$$
\mu_{e}=\frac{e \tau}{m_{c}^{*}} .
$$

A typical room lemperature mobility for a quantum well sample is about $7,000 \mathrm{~cm}^{2} / \mathrm{V}$ $s e c$, which increases to $50,000 \mathrm{~cm}^{2} / v-s e c$ as the temperature is reduced to $77 \mathrm{~K}$. The scattering time, as given by the above formula, is found to be about 0.25 picoseconds for the room temperature sample and about 1.8 picoseconds for the low temperature sample. If the decay of the upper state is assumed to be exponential then the lifetime will lead to a broadening with a Lorentzian lineshape and a FWHIM of $h / \pi r$. The linewidth increase for a room temperature sample due to lifetime is about $5.3 \mathrm{meV}$. The low temperature increase is about $0.69 \mathrm{meV}$ by the same calculation.

Unfortunately, the mobility lifetime is only loosly related to the ifelime of the upper quantum well state. The two relaxation processes are related in that they both involve a scattering of a conduction band electrc.s. The similarity ends here and the differences are many. The mobility is determined by the relaxation of the average momentum of an electron distribution back to the rest frame of the crystal, which requires in elastic scattering. Furthermore, the mohility of a quantum well is increased by the tendancy of a conduction band elect: $:$ not to scatter between quant.um well states, but instead scatter only within the plane of a single quantum state. But the scattering rate which determines the QWEST lifetime is probably an elastic transition between quantum well states, in contrast to both of the processes determining the mobility lifetime. Elastic scattering is generally 
much faster than inelastic scattering, and thus the mobilty determined rate is too slow on this grounds. But the scattering rate between quantum well states, which determines the QWEST lifetime, is slower than the scattering rate within the plane of single quantum well state, which determines the mobility lifetime. So the mobilty lifetime could easily be many times larger or smaller than the QWEST lifetime.

Attempts have been made to measure this lifetime in quantum wells with the newly developed femtosecond lasers.|27-80] Unfortunatly, these experiments, in spite of their resolution and sophistication, do not quite measure the quantities of interest to the QWEST. The highest resolution study performed of these references is that of D. J. Erskine, A. J. Taylor, and C. L. Tang[20], in which relaxation times as small as 30 femtoseconds are measured for the quantum well samples. But they used a $2.0 \mathrm{eV}$ photon as as the excitation of the upper states, leaving the electron with an excess energy of about $0.5 \mathrm{eV}$ for the room temperature measurement. An electron at this high an energy can elastically scatter from the $\Gamma$ symmetry point in the band diagram to the $L$ symmetry states, which have a band edge about $310 \mathrm{meV}$ above the direct band edge at the $\Gamma$ symmetry point. Since our quantum well energies are less than $310 \mathrm{meV}$ ahove the band edge, this scattering does not occur in our quantum wells. The optical phonon scattering was calculated in the same paper[20] have about an order of magnitude lower scattering rate, which would give a number similar to the numbers based on the mobility, but this rate was not measured.

An excellent measurement of the relaxation time of the electron gas temperature to the lattice temperature over many picoseconds under various exciatations and temperatures has been made by two groups. The first is that of C. V. Shank, R. L. Fork, R. Yen, J. Shah, B. I. Green, A. C. Gossard, and C. Weisbuch at Bell Laboratories. [27] The second is Z. Y. Xu and C. L. Tang at Cornell University. But of interest to the QWEST upper state lifetime is a different quantity, that of the 
relaxation time of the electrons among themselves, known to be a much faster phenomena. [27]

One of the measurements of D. J. Erskine, A. J. Taylor, and C. L. Tang, ${ }^{\text {[30] }}$ is of special interest since this experiment does measure the relaxation time of the electrons among themselves by viewing how quickly electrons excited at $0.5 \mathrm{eV}$ above the band edge return to the band edge. This measurement has the same problems as that of reference 29 in that at this high an excitation energy, the electron's dominant scattering mechanism is the $\Gamma-\mathrm{L}$ intervalley scattering mentioned above. But unlike the measurement of reference 29 , this ${ }^{[30]}$ experiment is looking at the lower energy states as it excites the upper states. So the electrons, which may initially scatter to other valleys, eventually must return to the $\Gamma$ valley as they cool. The rate of intervalley scattering is found ${ }^{[20]}$ to be in the 30 to 60 fimtosecond time scale. Thus we expect this ineasurement to be of some utility since the electrons quickly return to the valley of interest. The relaxation time measured for the quantum wells at room temperature was about 1 picosecond. However this measurement was made with an excitation density of $2 \times 10^{19} / \mathrm{cm}^{2}$ and our quantum wells are doped to a density of $4 \times 10^{17} / \mathrm{cm}^{2}$. Furthermore the electrons excited via the QWEST are only about $130 \mathrm{meV}$ above the lowest energy state, whereas the measurement ${ }^{30 \mid}$ is from electrons which start about $500 \mathrm{meV}$ ebove the lowest energy state. As such the QWEST lifetime could be significantly different.

In view of the uncertainties of the above description and meascrements and their relation to the upper quantum well relaxaition time, little can be definitively soid about the QWEST lifetime. However the above observations lead us to expect a relaxation time between 0.2 and 1.0 picoseconds for the quantum well at room temperature. This would lead to a linewidth broadening between 1 and $6 \mathrm{meV}$. The scaling to lower temperatures is not known. If the scattering is primarily 
determined by phonon scattering, then the QWEST would almost certairly have a longer lifetime and smaller broadening as the temperature is reduced. However, if the scattering is determined by free electron scattering or scattering off of the walls, ions or other fixed source, then the scattering rate for the upper state will probably remain constant with temperature reduction. The latter case is suspected to be much more probable.

\section{Well width inhomogeniety}

A variation in the width of the well can lead to a change in the energy of the quantim well states. As an example we see in Fig. IV.2 that the variation in QWEST energy with well thickness is roughly linear with a slope of $1.1 \mathrm{meV}$ per $\AA$ of well width. Fortunately, this well width variation can be measured directly from its broadening of a two-dimensional exciton energy (see chapter II., section D.). This measurement has been made as a function of the substrate growth temperature in the Molecular Beam Epitaxy chamber[31] and found to have a minimum width of $1.0 \mathrm{meV}$ at a growth temperature of about $690^{\circ} \mathrm{C}$ (see Fig. IV.6). Our samples were grown at a temperature of about $660^{\circ} \mathrm{C}$. The linewidths of the exciton at this temperature are about $1.4 \mathrm{meV}$. Since the well thickness used in this measurement ${ }^{[31]}$ is $200 \AA$, the linewidth of our 65 to $100 \AA$ wells with the same size variation in thickness is about $1.6 \mathrm{meV}$. 


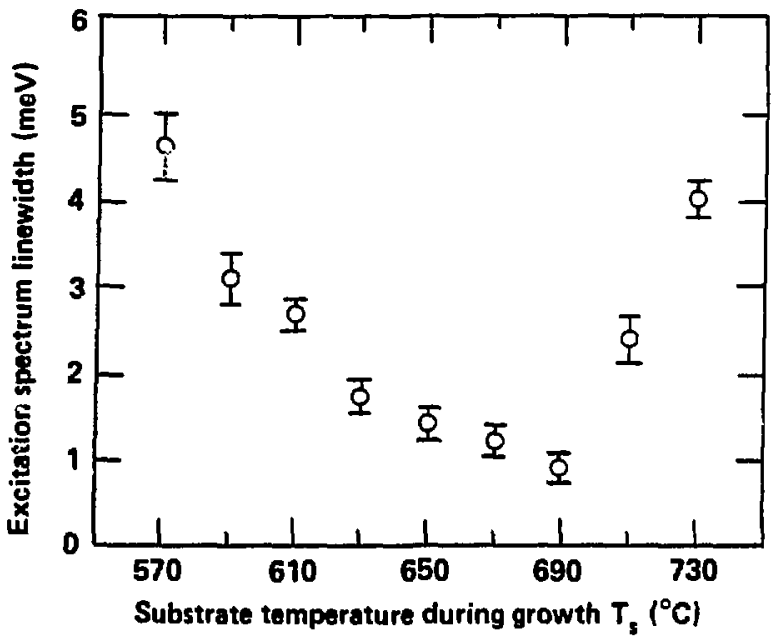

Figure IV.6: A plot of the 2-D exciton linewidth of various quantum well samples as a function of substrate growth temperature (from Ref. [31]).

In addition to perturbations on a microscopic scale, one can also have syste.. atic gradients in well thickness across the surface of a quantum well sample. These usually arise from a gradient in the rate of the molecular flux on the surface of the sample since the molecular sources are usually off axis from the center of the sample. The variation in flux rate is about 20 to $30 \%$ across the diameter of a 2 inch wafer. The wafer is sometimes rotated to average out these variations. This was not done for the quantum wells grown since the maximum rotation rate possible for the growth apparatus did not permit several rotations during the 30 seconds needed to grow a single well of $65 \AA$. It will be shown later that the infrared beam probing the QWEST has a dimension of 3 by $11 \mathrm{~mm}$. The variation in thickness will then be that of the wafer times $3 / 51$ or $11 / 51$, depending on the direction scanned. For a $30 \%$ variation in thickness across the wafer, the variation in thickness across the beam pattern is about 1.8 to $6.5 \%$ of the thickness grown. We note this 
variation corresponds to a variation in thickness of about 1.1 to $4.2 \AA$ for a $65 \AA$ well. This in turn gives a linewidth increase or about 1.2 to $4.6 \mathrm{meV}$, depending on the orientation of the crystal thickness gradient to the infrared beam pattern. If we add this inhomogeneous term to that above, the inhomogeneous broadening is anticipated to be in the range of 2.8 to $6.2 \mathrm{meV}$. The crystal is oriented so as to acheive the narrowest linewidth so the smaller number will be used. The variation of linewidth with crystal orientation will be examined later.

One of the quantum wells we tested was inadvertantly rotated at a very slow speed during growth, so each section of the sample received a distribution of well widths within the 50 quantum well stack which was grown. The measured spectra was seen to be broadened about an additional $10 \mathrm{meV}$, corresponding to a thickness variation of about 10 to $15 \%$ among the wells. The spectra also became very strongly dependent on the beam pattern on the surface. This sample, B-331, will be discussed in some detail in the next chapter.

\section{Sum of the broadening effects}

The suspected broadening terms of the QWEST linewidth are tabulated for a $65 \AA$ well at room temperature in Table IV.1.

\section{Table IV.1}

Broadening effects for a $65 \AA$ quantum well at $300 \mathrm{~K}$.

\begin{tabular}{ll} 
Density of QWEST states & $7.5 \pm 2.0 \mathrm{meV}$ \\
Lifetime broadening & $3.0 \pm 3.0 \mathrm{meV}$ \\
Inhomogeneous well width & $3.0 \pm 1.5 \mathrm{meV}$ \\
\hline Total & $8.7 \pm 3.3 \mathrm{meV}$
\end{tabular}

The total assumes the linewidths add as the square root of the sum of squares of the individual linewidths. If we reduce the temperature of a $65 \AA$ well to $35 \mathrm{~K}$, 
the broadening terms change as shown in Table IV.2.

\section{Table IV.2}

Broadening effects for a $65 \AA$ quantum well at $35 \mathrm{~K}$.

\begin{tabular}{ll} 
Density of QWEST states & $2.4 \pm 0.6 \mathrm{meV}$ \\
Lifetime broadening & $3.0 \pm 3.0 \mathrm{meV}$ \\
Inhomogeneous well width & $3.0 \pm 1.5 \mathrm{meV}$ \\
\hline Total & $4.9 \pm 3.0 \mathrm{meV}$
\end{tabular}

The bandwidth can be seen to have large uncertainties as to the mechanisms involved, particuarly in the lifetime. The $82 \AA$ well is clearly broadened by an inadvertant variation of weil thickness from well to well during growth of this sample as will be discussed later. Precise measurements of lifetime, exciton linewidths, and quantum well energies as a function of well thickness (to determine band nonparabolicity ) may eventually lead to a better understanding of the precise role of each of these mechanisms. 


\section{E. Energy Shift Mechanisms}

The energy of the quantum well states was calculated in section $B$. from the potential of the crystal in which it was contained. However this calculation neglected any external fields and the coulomb potential arising from the electrons themselves. An external field can be shown to have only a small effect on the QWEST energy and is not discussed. In contrast, the electron self-repulsion can have a noticable effect on the quantum well energy. This effect is dependent on electron deusity and lowers the QWEST encrgy by about $2 \mathrm{meV}$ at the densities we use of $4 \times 10^{11} / \mathrm{cm}^{2}$. Another mechanism which shifts the observed energy spectrum is the dynamic interaction of the probing field with the electron plasma of the quantum well. This causes the absorption peak to appear at a higher energy than the true quantum energy difference of the states. For the density of electrons and well thicknesses we use, the observed energy absorption peak is about $3 \mathrm{meV}$ above the quantum well energy difference.

\section{Coulomb interaction}

The electrons within the well region create an electrostatic potential, $\Phi$, which interacts with both upper and lower quantum well states. This potential can be found from Maxwell's equations in one dimension;

$$
\frac{\partial^{2}}{\partial z^{2}} \Phi=\frac{-p(z) e^{2}}{\epsilon} .
$$

We can use the solution to the wavefunctions in section $B$ to find the electron distribution as a function of $z$. This probability distribution is integrated twice to find the potential using equation (IV.64). The effect of this potential on the quantum well states is found from first order perturbation theory.

The integrations over the wavefunctions of section $B$ and the subsequent perturbation calculations are very lengthy and fairly tedious. But all understanding 
and a fairly good estimate of the effect can be found by using infinite well envelope states. This estimate calculation is presented here and later compared with the results of the accurate calculation.

The wavefunctions of the lower and upper envelope states for the infinite well are given by

$$
\psi_{l}=\sqrt{\frac{2}{L_{z}}} \cos \left(k_{q} z\right)
$$

and

$$
\psi_{u}=\sqrt{\frac{2}{L_{z}}} \sin \left(2 k_{q} z\right)
$$

respectively, where the origin of the well is taken at the center of the well and as before, $k_{q}=\pi / L_{z}$ and the edges of the well are at $\pm L_{z} / 2$. The electrons are assumed to all reside in the lower energy state. The electron surface density is taken to be $\rho_{\text {. }}$. The density distribution, $\rho(z)$ then becomes

$$
\rho(z)=\frac{2 \rho_{8}}{L_{z}} \cos ^{2}\left(k_{q} z\right)
$$

Insertion of this density into equation (IV.64) yields after the first integration the result

$$
\frac{\partial}{\partial z} \Phi=-\frac{\rho_{\theta} e^{2}}{\epsilon} \frac{2}{L_{z}}\left[\bar{z}+\frac{\sin \left(2 k_{q} z\right)}{4 k_{q}}\right]-E_{0}
$$

where $E_{0}$ is the constant of integation and is equal to the electric field at $z=0$. In the absence of an external feld, this term can be seen to be equal to zero by reason of symmetry. Note that even if this term were nonzero, by application of an external field or by absence of symmetry, the effect on the quantum well energies 
can be seen to cancel to first order. If we let $E_{0}$ be zero, the integral of equation (IV.67) gives an expression for the potential of

$$
\Phi=-\frac{\rho_{\theta} e^{2}}{\epsilon} \frac{2}{L_{z}}\left[\frac{z^{2}}{4}-\frac{\cos \left(2 k_{q} z\right)}{8 k_{q}^{2}}\right]
$$

where we have taken the arbitrary constant of the potential to be zero.

Using the above expression for the electrostatic potential of the quantum well, the energy shift of the two quantum well states can now be calculated using first order perturbation theory. Perturbation theory gives the first order correction to the energy of the states, $\Delta F$ as

$$
\begin{aligned}
\Delta E & =(\psi|\Phi| \psi) \\
& =\int_{-L_{z} / 2}^{L_{z} / 2} \psi^{*} \Phi \psi d z .
\end{aligned}
$$

The energy shift of the lower envelope state now becomes

$$
\begin{aligned}
\Delta E_{l} & =-\int_{-L_{z} / 2}^{L_{z} / 2} \frac{\rho_{\theta} e^{2}}{\epsilon}\left(\frac{2}{L_{z}}\right)^{2}\left[\frac{z^{2}}{4}-\frac{\cos \left(2 k_{q} z\right)}{8 k_{q}^{2}}\right] \cos ^{2}\left(k_{q} z\right) d z \\
& =-\frac{\rho_{\theta} e^{2}}{\epsilon} \frac{L_{z}}{2 \pi^{3}} I_{1}
\end{aligned}
$$

where

$$
I_{1}=\int_{-\pi / 2}^{\pi / 2}\left[2 \theta^{2}-\cos (2 \theta)\right] \cos ^{2}(\theta) d \theta
$$

The integral $I_{1}$ is evaluated in Appendix A, part 1. The perturbation energy now becomes

$$
\begin{aligned}
\Delta E_{i} & =-\frac{\rho_{s} \mathrm{e}^{2}}{\epsilon} \frac{L_{z}}{2 \pi^{3}}\left[\frac{\pi^{3}}{12}-\frac{3 \pi}{4}\right] \\
& =-\frac{\rho_{8} \mathrm{e}^{2}}{\varepsilon} \frac{L_{z}}{8}\left[\frac{1}{3}-\frac{3}{\pi^{2}}\right]
\end{aligned}
$$


The upper state energy shift is calculated similarly. Tue energy shift of the upper state is found from first order perturbation theory as

$$
\begin{aligned}
\Delta E_{u} & =-\int_{L_{z} / 2}^{L_{z} / 2} \frac{\rho_{\varepsilon} e^{2}}{\epsilon}\left(\frac{2}{L_{z}}\right)^{2}\left[\frac{z^{2}}{4}-\frac{\cos \left(2 k_{q} z\right)}{8 k_{q}^{2}}\right] \sin ^{2}\left(2 k_{q}\right) d z \\
& =-\frac{\rho_{q} e^{2}}{\epsilon} \frac{L_{z}}{2 \pi^{3}} I_{2}
\end{aligned}
$$

where the integral $l_{2}$ is equal to

$$
I_{2}=\int_{-\pi / 2}^{\pi / 2}\left[2 \theta^{2}-\cos (2 \theta)\right] \sin ^{2}(2 \theta) d \theta
$$

and is evaluated in Appendix A, part 2. The upper state energy is then shifted by an amount

$$
\begin{aligned}
\Delta E_{u} & =-\frac{\rho_{g} e^{2}}{\epsilon} \frac{L_{z}}{2 \pi^{3}}\left[\frac{\pi^{3}}{12}-\frac{\pi}{8}\right] \\
& =-\frac{\rho_{g} e^{2}}{\epsilon} \frac{L_{z}}{8}\left[\frac{1}{3}-\frac{1}{2 \pi^{2}}\right] .
\end{aligned}
$$

The energy shift of the QWEST, $\Delta E_{Q}$ can now be calculated from the above two expressions;

$$
\begin{aligned}
\dot{\Delta} E_{Q} & =\Delta E_{u}-\Delta E_{l} \\
& =-\frac{\rho_{\theta} e^{2}}{\epsilon} \frac{L_{z}}{2}\left[\frac{-1}{2 \pi^{2}}+\frac{3}{\pi^{2}}\right] \\
& =-\frac{\rho_{g} e^{2} L_{z}}{\epsilon} \frac{5}{16 \pi^{2}} .
\end{aligned}
$$

This energy shift is seen to be proportional to product of the well thickness and the surface charge density. If we let $L_{z}$ and $\rho_{0}$ be $80 \AA$ and $4 \times 10^{11} / \mathrm{cm}^{2}$ and use $\epsilon=13 \varepsilon_{0}$, then $\Delta E_{Q}=-1.4 \mathrm{meV}$. A more extensive derivation including the envelope tunneling into the barrier and the solutions for $k_{q}$ gives an energy shift 
of $-2.1 \mathrm{meV}$. The larger number is expected for the larger wavefunction of the true envelope states relative to those for the infinite well. The energy shift of other size wells scale proportionally so a $65 \AA$ thick well would have an entrgy shift of -1.7 $\mathrm{meV}$.

\section{Dynamic interaction with the free electron plasma}

The effect of an external electric field on the quantum well states is described by its value at the location of the well. But the potential at the location of the electron in the quantum well is not neccesarialy that determined from an external field, depending on the shielding caused by the electron gas. This shielding has been shown to cancel the peak absorption resonance at what would be expected to be absorption energy of the transition[32-34]. The effect was first described by Chen, Chen, and Burstein[34] and then formulated in a readily understandable quantum argument by Allen, Tsui, and Vinter ${ }^{321}$. The quantum argument was then further developed by Dahl and Sham. [3s] The validity of this effect was confirmed by inelastic light scattering ${ }^{[10]}$ in $\mathrm{GaAs}$ quantum wells as discussed in chapter II, section F.

A simple explaination in classical terms for this effect can be given as follows. Suppose the quantum well is placed in an external electric field of frequency $\omega$. Then the dielectric constant of the medium near a strong resonance at $\omega_{0}$ can be written as

$$
\epsilon(\omega)=\epsilon_{m}+\frac{\Omega_{p}^{2} f}{\omega_{0}^{2}-\omega^{2}+i \gamma \omega}
$$

where $\Omega_{p}^{2}=n e^{2} / m_{e} \epsilon$ is the plasma frequency squared, $\gamma$ is a resistive loss term of the resonance, $n$ is the three dimensional electron density, and $f$ is the oscillator strength. The dielectric strength from all other transitions are assumed to be slowly varying for this description near $\omega_{0}$, and are included by the constant $\varepsilon_{m}$. The power absorbed per unit volume, PA, is now given by [15] 


$$
P A=\frac{\epsilon_{0}}{2}\left|E_{i}\right|^{2} \omega \operatorname{Im}(-\epsilon(\omega))
$$

where $E_{i}$ is the internal feld of the crystal. Using this formula we usually expect a maximum absorption peak at the resonant frequency of $\omega_{0}$, as occurs from simple substitution of equation (IV.77) into equation (IV.78). But for strong resonances, this expression must allow for the differences between the external and internal field which varies as a function of frequency. For instance, the field which interacts with the QWEST is normal to the surface of the quantum well. Thus the boundary condition gives the relation $E_{i}=E_{e} / \epsilon(\omega)$, where $E_{i}$ and $E_{e}$ are the internal and external electric fields, respectively. From equation (IV.77), we see that

$$
\frac{1}{|\epsilon(\omega)|^{2}}=\frac{1}{\epsilon_{m}^{2}} \frac{\left(\omega_{0}^{2}-\omega^{2}\right)^{2}+\omega^{2} \gamma^{2}}{\left(\Omega_{p}^{2} J / \epsilon_{m}+\omega_{0}^{2}-\omega^{2}\right)^{2}+\omega^{2} \gamma^{2}} .
$$

The plasma frequency term can be written as $\Omega_{p}^{2} J / \epsilon_{m}=\Omega_{p}^{* 2}$, where $\Omega_{p}^{* 2}$ is the effective plasma frequency obtained by using the crystal dielectric strength and effective mass in the above expression for plasma frequency. The numerator of equation (IV.79) can be seen to cancel the denominator of the imaginary part of $f(\omega)$ as given in equation (IV.77). The power absorbed then becomes

$$
P A=\frac{\Omega_{p}^{2} \int \gamma \omega^{2}}{\left(\omega_{0}^{2}+\Omega_{p}^{*}-\omega^{2}\right)^{2}+\gamma^{2} \omega^{2}} \frac{\epsilon_{0}}{2 \epsilon_{m}^{2}}\left|E_{e}\right|^{2}
$$

and now is maximized at a different resonant frequency of $\omega_{0}^{*}$, given by

$$
\omega_{0}^{2}=\omega_{0}^{2}+\Omega_{p}^{\prime 2}
$$

as stated earlier in equation (II.29). The theory of Allen, Tsui, and Vinter [32] develops an expression from the quantum dynamics of the interaction and has the following result for the power absorbed per unit area; 


$$
P A S=\frac{\rho_{\theta} e^{2}}{\hbar \omega_{0}} \frac{\langle 1|z| 2\rangle^{2} \omega_{0}^{2} \omega^{2} \gamma}{\left(\omega_{0}^{2}+\omega_{p}^{2}-\omega^{2}\right)^{2}+\omega^{2} \gamma^{2}}\left|E_{e}\right|^{2}
$$

where

$$
\begin{gathered}
\omega_{p}^{2}=\frac{2 N_{e} \mathrm{e}^{2}}{c \hbar} \omega_{0} S_{22} ; \\
S_{22}=\int_{-\infty}^{\infty}\left[\int_{-\infty}^{z} \psi_{2}\left(z^{\prime}\right) \psi_{1}\left(z^{\prime}\right) d z^{\prime}\right]^{2} d z
\end{gathered}
$$

where $\psi_{n}$ is the envelope wavefunction of the $n^{\prime}$ th envelope state and $\rho_{\theta}$ is the electron density per unit area. If we let the plasma frequency be the same, then the two expressions (IV.80) and (IV.8\%) are identical with the aid of the definition of oscillator strength in equation (III.14) and letting $P A S$, the absorption per unit area, be converted to an absorption per unit volume by the ratio of $n$ to $\rho_{g}$.

The expression for $\omega_{p}^{2}$ in equation (IV.83) can be related to a three dimensional plasma frequency if we use the infinite barrier envelope states of chapter III. The frequency $\omega_{0}$ becomes $\omega_{0}=3 \hbar k_{q}^{2} / 2 m^{*}$ and the integral $S_{22}$ becomes $S_{22}=\left(5 / 9 \pi^{2}\right) L_{z}=0.0563 L_{z}$. The plasma frequency squared then becomes $\omega_{p}^{2}=$ $\left(N_{8} / L_{z}\right) e^{2} / \mathrm{cm}^{*} \times 5 / 3$. This expression shows qualitatively how the expression of equation (IV.83) is about $5 / 3$ times the three dimensional plasma frequency.

The energy shift is calculated using the expression for the plasma frequency in equation (IV.83). The value of $S_{22}$ is found emperically(10) to be about $0.072 L_{z}$. This is larger than the value of $0.056 L_{z}$ found for an infinite well, as expected, since the wavefunctions for a finite barrier extend further into the barrier region. We now calculate the energy shift for several wells for a doping concentration of $4 \times 10^{11} / \mathrm{cm}^{2}$. The energy used in equation (IV.83) is that calculated in section $B$ with the assumption of continuity of the envelope derivitive at the well boundary 
and labeled $E_{Q 0}$. The shifted energy is labeled $E_{Q}^{*}$ and the plasma frequency times $\hbar$ is labeled $E_{p l}$. Note the infinite well expression gives a value of $E_{p l}$ of $51 \mathrm{meV}$ for a $65 \AA$ quantum well. On the other hand, equation (IV.83) gives a value of $28.5 \mathrm{meV}$ for $E_{p l}$. The infinite well thus gives a poor approximation for the plasma frequency of the two-dimensional well.

The calculated energies and shifts are now tabulated in Table IV. 3 for three well thicknesses of interest. The theoretical absorption resonance energy, $E_{A}$, is found by adding the coulomb energy shift of the previous part to $E_{Q}^{*}$.

\section{Table IV.3}

The QWEST energy and energy shifts for quantum wells with thicknesses of 65,82 and $92 \AA$. Energies are in units of $\mathrm{meV}$.

\begin{tabular}{cccccc} 
Thickness & $E_{Q 0}$ & $E_{p l}$ & $E_{Q}^{*}$ & $\Delta E_{Q}$ & $E_{A}$ \\
\hline $65 \AA$ & 141 & 29.8 & 144.1 & -1.7 & 142.4 \\
$82 \AA$ & 116 & 30.5 & 118.8 & -2.1 & 117.8 \\
$92 \AA$ & 103 & 30.4 & 107.4 & -2.4 & 105.0
\end{tabular}

The dynamic electron plasma interaction is seen to nearly cancel the electrostatic coulomb interaction, anc the net energy shift is about $1.0 \mathrm{meV}$ upward for the well widths and doping densities used here. This small an energy shift is below the accuracy of the initial quantum well energies. 


\section{F. Optical Properties}

The optical properties of the QWEST are of special interest because of the transition's high oscillator strength, narrow bandwidth, and fast decay time. In this section, several optical properties are calculated for the QWEST. These include the index of refraction, the absorption rate, and the nonlinear index of refraction, usually called $n_{2}$.

\section{Index of refraction}

The electric susceptibility of a dielectric medium, $\chi$, is defined by $P=t_{0} \times E$ where $P$ and $E$ are the polarization of the medium and the applied electric field, respectively. The electric susceptibility for an dipole transition between two quantum states is given by Yarivisis as

$$
\begin{aligned}
& x^{\prime}(\omega)=\frac{\mu^{2} T_{2} \Delta N_{0}}{\epsilon_{0} \hbar} \frac{\left(\omega_{0}-\omega\right) T_{2}}{1+\left(\omega-\omega_{0}\right)^{2} T_{2}^{2}+4 \Omega^{2} T_{2} \tau}, \\
& \chi^{\prime \prime}(\omega)=\frac{\mu^{2} T_{2} \Delta N_{0}}{\epsilon_{0} \hbar} \frac{1}{1+\left(\omega-\omega_{0}\right)^{2} T_{2}^{2}+4 \Omega^{2} T_{2} \tau},
\end{aligned}
$$

where the dielectric response has been seperated into real and imaginary parts, where $x=x^{\prime}+i x^{\prime \prime}$. The linear and nonlinear index of refraction is determined from $x^{\prime}$ and the absorption of light and its saturation are determined by $x^{\prime \prime}$. The time for the upper and lower quantum states to loose phase coherence from one another is called $T_{2}$. The relaxation time for the quantum states to return to their equilibrium distribution between the lower and upper states is called $r$. The "precession" frequency $\Omega$ is defined by $\Omega \equiv \mu E_{0} / 2 \hbar$ where $E_{0}$ is the amplitude of the driving field at a frequency $\omega$. The equilibrium population difference, $\Delta N_{0}$, between the upper and lower states is taken to be the doping density since at the energies and temperaiures we use, only small fraction of electrons are in the upper 
state. The polarization obtained from the use of a surface density must be averaged over the distance between wells to obtain the average polarization. Therefore, one can generally use an averaged density for $\Delta N_{0}$. The dipole, $\mu$, is the same dipole calculated previously in section $\mathrm{C}$. The resonant frequency, $\omega_{0}$, is equal to the energy difference of the two quantum states over $\hbar$.

The index of refraction of the medium is determined from square root of the dielectric constant, $\epsilon$, which is related to $\chi$ by $\epsilon=\epsilon_{m}+\epsilon_{0} \chi$, where $\epsilon_{m}$ is the dielectric constant of all other transitions in the frequency domain of interest. The refractive index of the media from all other trasitions, $n_{0}$, is given by $n_{0}=\sqrt{\epsilon_{m}}$. The index of refraction change, $\Delta n$ due to the transition at $\omega_{0}$ is then given by

$$
\Delta_{n}=\frac{x^{\prime}}{2 n_{0}}
$$

If we assume that the dipole is $17 \mathrm{e} \AA$ and $T_{2}$ is equal to 0.2 picoseconds, then $\Delta n=2.5 \times 10^{-18} \mathrm{~cm}^{3} \Delta N_{0} g(\omega)$, where $g(\omega)$ is the lineshape factor,

$$
g(\omega)=\frac{\left(\omega_{0}-\omega\right) T_{2}}{1+\left(\omega-\omega_{0}\right)^{2} T_{2}^{2}}
$$

\section{Nonlinear refraction}

A nonlinear refraction index, $n_{2}$, is disined by

$$
n \equiv n_{0}+n_{2} I
$$

where $n_{2}$ is seen to be the linear constant between the iadex of refraction and the incident optical intensity, $I$. The expression for this term can be found from equation (IV.84a) by expanding the $\Omega^{2}$ term in the denominator to first order in this term. This first order term of $\chi^{\prime}$ in $\Omega^{2}$ is labeled $\chi^{(3)} E_{0}^{2}$ since the polarization is now proportional to $E_{0}^{3}$ instead of $E_{0}$. Expansion of equation (IV.84a) now gives 


$$
\chi^{(3)}(\omega) E_{0}^{2}=\frac{2 \mu^{4} \eta_{0} T_{2}^{3} \tau\left(\omega-\omega_{0}\right)}{n_{0} \epsilon_{0} \hbar^{3}\left(1+\left(\omega-\omega_{0}\right)^{2} T_{2}^{2}\right)^{2}} \Delta N_{0} \frac{n_{0} E_{0}^{2}}{2 \eta_{0}}
$$

where $\eta_{0} \equiv \sqrt{\mu_{0} / \epsilon_{0}} \approx 377 \mathrm{ohm}$ and $\mu_{0}$ is the magnetic permeability of free space. This nonlinear coefficient can be related to the nonlinear index of refraction by first using the relation between the amplitude of an oscillating electric field in a dielectric medium and the optical intensity in the medium;

$$
I=\frac{n_{0} E_{0}^{2}}{2 \eta_{0}}
$$

Now using equations (IV.86) through (IV.88) we obtain

$$
n_{2}=\frac{\chi^{(3)}(\omega) E_{0}^{2}}{2 n_{0} T}=\frac{e^{4}\langle z\rangle^{4} T_{\overline{2}}^{\prime \prime} \tau \Delta N_{0}}{\epsilon_{0}^{2} c \hbar^{3} n_{0}^{2}} \frac{\left(\omega-\omega_{0}\right) T_{2}}{\left(1+\left(\omega-\omega_{0}\right)^{2} T_{2}^{2}\right)^{2}}
$$

where the dipole $\mu$ has been set equal to $e\langle z\rangle$. We now define the lineshape factor on the right by $g^{(3)}(\omega)$, given by

$$
g^{(3)}(\omega) \equiv \frac{\left(\omega-\omega_{0}\right) T_{2}}{\left(1+\left(\omega-\omega_{0}\right)^{2} T_{2}^{2}\right)^{2}}
$$

If we now take $(z)=17 \AA, \Delta N_{0}=4 \times 10^{17} / \mathrm{cm}^{3}, n_{0}=3.27, \tau=4$ picoseconds, and $T_{2}=0.5$ picoseconds we then obtain

$$
n_{2}=0.96 \times 10^{-4} \frac{c m^{2}}{\text { Watt }} g^{(3)}(\omega)
$$

The magnitude of the nonlinear coefficient depends on how close the light is to the resonance. We find the maximum occurs when $\left(\omega-\omega_{0}\right) T_{2}$ equals $1 / \sqrt{3}$, at which point $g^{(3)}(\omega)=0.325$. The nonlinear coefficient then has a maximum value of $3.11 \times$ $10^{-5} \mathrm{~cm}^{2} /$ Watt. 


\section{Absorption rate}

The absorption rate, $x(\omega)$, for a collection of dipole oscillators is the rate at which light of a given intensity $I$ wili decay exponentially into a medium. This rate is given by Yariv[15] as

$$
\gamma(\omega)=-\frac{k_{0} x^{n}}{n_{0}}
$$

where $k_{0}$ is the free space wavevector of the light. This equation becomes with the aid of equation (IV.84b),

$$
\gamma(\omega)=-\frac{2 \pi}{n_{0} \lambda_{0}} \frac{\mu^{2} T_{2} \Delta N_{0}}{c_{0} \hbar} o^{\prime}(\omega)
$$

where $\lambda_{0}$ is the free space wavelength and the absorption lineshape factor, $g^{\prime}(\omega)$ is defined by

$$
g^{\prime}(\omega)=\frac{1}{1+\left(\omega-\omega_{0}\right)^{2} T_{2}^{2}} .
$$

This absorption constant can be put into a simpler form with the aid of the oscillator strength, $f$. Using the oscillator strength definition of equation (III.14), one obtains

$$
\gamma(\omega)=\Delta N_{0} \frac{e^{2} T_{2}}{2 \epsilon_{0} m_{e} c n_{0}} \int g^{\prime}(\omega) .
$$

We now take value of $\lambda_{0}$ as 8.15 microns, which is the wavelength of a photon with energy of $0.152 \mathrm{meV}$. We also let $\mu-17 \mathrm{e}-\AA, n_{0}=3.27, T_{2}=0.5$ picoseconds, and $\Delta N_{0}=4 \times 10^{17} \mathrm{~cm}^{-i} \quad \sim$ absorption constant then becomes $\gamma(\omega)=3.8 \times$ $10^{4} \mathrm{~cm}^{-1} g^{\prime}(\omega)$. The cross section, $\sigma$, defined as the ratio of the absorption constant to the population difference, is approximately $\sigma=1 \times 10^{-13}$.

We remark that the all of the above optical properties are proportional to $\Delta N_{0}$, the average dopant density. In our numerical estimates we used a value of 
$4 \times 10^{17} \mathrm{~cm}^{-3}$ for this quantity. In the next chapter we will see that this doping density only exists within a $100 \AA$ region, with the average undoped spacing between each of the doped regions being from 400 to $100 \AA$, depending on whether buffer layers are inserted or not. Thus the lower state density, when averaged over the entire region, will become equal to 20 to $50 \%$ of the doping density in the doped region. If we wish for the above numerical estimates, which are linear in lower state density, to apply we must multiply them by .2 to .5 respectively. These optical quantities also only apply to optical rays propagating entirely within the epitaxial region containing the quantum wells in a regular array. Obviously, outside the region containing the quantum wells no QWEST optical effects can occur. Also, the QWEST has a nonzero dipole only for one polarization of the electric field. The interaction of infrared light of other polarizations with the QWEST must be reduced by a factor including the inner product of this polarization with tue QWEST dipole, usually taken to be in the $z$ direction. The effect of this lower coupling is equivilent to lowering the dipole in the above optical quantities by the cosine of the angle between the infrared polarization and the QWEST dipole. The next chapter will show how the above optical properties are to be modified when the optical beam is propagating at an angle to a number of quantum wells, as occurs for our measurements. 


\section{V.QWEST Absorption Experiments}

In this chapter we describe the experiments performed to observe the QWEST. We present the results of those experiments and compare these results with the theory of chapter IV.

\section{A. Mechanics of the Optical Probe}

The optical experiments were oriented towards observing absorption of infrared radiation by the QWEST. The primary goal was simply to prove that a dipole transition between the envelope states truly exists in a real material. It required years of effort to see this effect, during which many serious questions arose about both its theoretical existence and how close real quantum wells approximated a theoretical two dimensional system. For instance, if the quantum well walls were sufficiently rough then scattering could destroy the effect either by increased lifetime broadening or by lack of conservation of transverse momentum wavevector. As will be shown, neither is the case. The primary difficulty in the observation of the effect was found to simply be a trappi'ig of all free electrons by deep energy states, most likely at the interfaces.

The second goal was to measure the energy of the QWEST. These energies could then be $c c$.pared with the theoretical predictions as a measure of understanding of the quantum well states. A third goal was to prove that the oscillator strength of the transition was as large as theory predicted and to obtain good agreement between the two. And finally, a measure of the linewidith would Indicate if $k$ vector conservation truly occured.

The lower quantum well state could be populated by either photopumping the state from the valence band or by doping the conduction band. The advantage of photopumping is the capability to vary the electron surface density by adjusting the pump power. The disadvantage is spatial inhomogeneity of the lower state 
population, transversly because of the laser beam profile and in depth because of attenuation of the optical beam. These variations would make an accurate measurement of oscillator strength very difficult. Also, it would require approximately 1 kilowatt of optical power to maintain an inversion density of $4 \times 10^{11} \mathrm{~cm}^{-2}$ per well, and this inversion is desired to be continots.

The infrared absorption measurement could use either an infrared laser or a spectrometer. The laser has the advantage in that it could be focused into a waveguide on the surface to couple to the QWEST with the proper polarization. In addition it could be pulsed to allow photopumping as a method of populating the lower state. The problem with using a laser is that it is not very tunable. A $\mathrm{CO}_{2}$ laser can be tuned from 9 to 11 micron wavelength and would be an ideal source for the absorption measurement if a QWEST resonance could be placed in the vicinity of the lasing tunability. However, it was felt that the chance of a successful measurement would be much better if a broad absorption spectrum could be observed. Not only would less demands be placed on the precision of the crystal growth and theory, but a surrounding spectrum could be observed as an example of types of phenomena which occur in the energy region. This approach proved invaluable.

Once it was felt a spectrometer measurement was advantageous, the choice of instuments eventually narrowed to a Hourier Transform Infrared Radiation (FTIR) spectrometer. These spectrometers are commonly used by chemists and readily available. Furthermore, the FTIR spectrometers are capable of measuring low absorption values with low noise and large spectral regions with fundamentally accurate calibration of frequencies.

The spectrometer used for these measurements was a Nicolet 7189 which incorporates its own 20 bit computer. The optical layout of the 7109 FTIR spectrometer 
is illustrated in Fig. V.1.

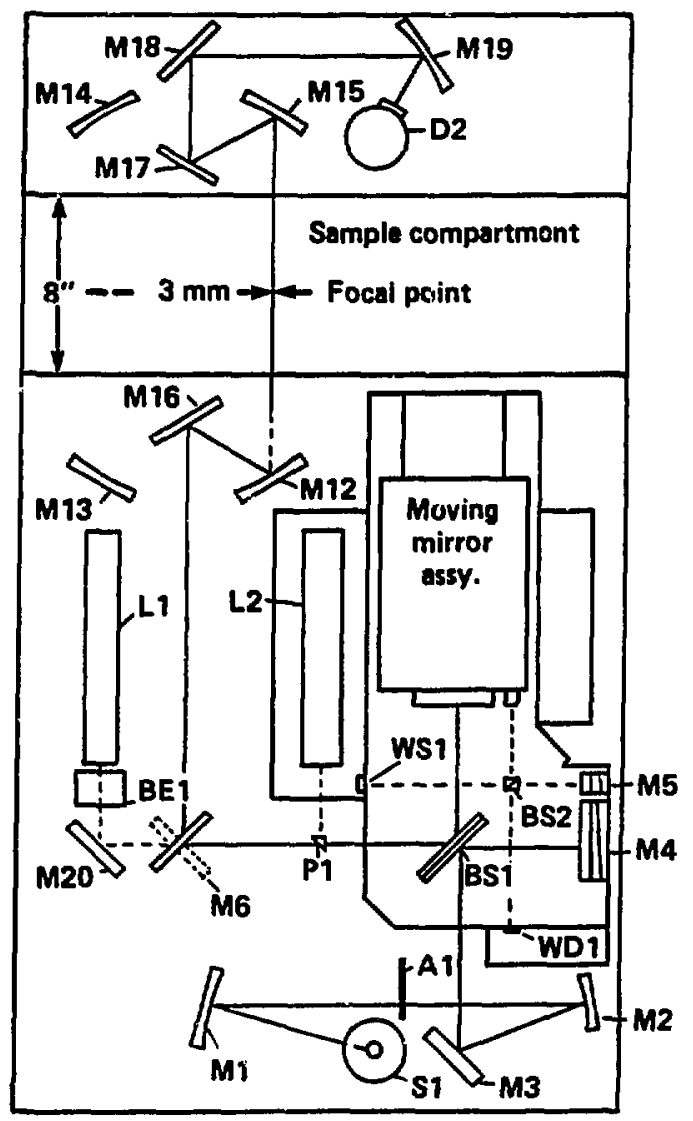

Figure V.1: Nicolet spectrometer configuration.

The legend is as follows: 
Si -Infrared Source

M1 -Spherical mirror

A1 -Aperture and chopper

M2 -Spherical mirror, 8.0" E.F.L.

M3 -Flat mirror

BS1 -Beamsplitter

BS2 - White light beamsplitter

M4 -Fixed mirror

M5 -White light mirror

P1 -Centerline laser prism

M6 -4-position flat mirror

M16 -2-position flat mirror

M17 -2-position flat mirror
M12 -Off-axis parabolic mirror, 9.3" E.F.L.

M13 -Off-axis parabolic mirror, 9.3" E.F.L.

M14 -Off-axis parabolic mirror, 9.3" E.F.L.

M15 -Off-axis parabolic mirror, 9.3" E.F.L.

M18 -Flat mirror

M19 -Off-axis parabolic mirror, 9.3" E.F.L.

L1 -Alignment laser

L2 -Centerline laser

M20 -Flat mirror

WS1 - White light source

WD1 - White light detector

BEl -Beam expander

D2 -HgCdTe detector and LN2 dewer

The interferometer operates as follows. Infrared light is emitted by source S1 and is then apertured by A1. The infrared light then enters a Michelson interferometer formed by beamsplitter BS1 and the two mirrors, M4 and a second movable mirror on an air bearing. The movable mirror slides back and forth on its mount, so that a given wavelength will have a sinusoidal variation in its intensity on the output of the interferometer at mirror M6. Each wavelength will have a different periodicity, so by looking at how each frequency component of an infrared beam differs on being transmitted through a sample or not, the absorption spectrum is obtajned. The fourier transform needed to convert the detected interferogram into a spectral curve is done digitally. The position of the moving mirror assembly is detected by laser L2, the centerline laser, which is also sent through the Michelson interferometer by a small prism which protrudes into the infrared beam path. The wavelength of the infrared light is ratioed to the HeNe lasel wavelength of laser L2 by 
simply comparing the relative ratio of cycles in the interferogram. Thus the infrared frequency calibration is very good and always within the frequency resolution of the measurement. The zero point of interferometer is found from a white light source, WS1, with its own interferometer formed by BS2 and detected by WD1. The resolution of a given measurement is determined by how far the moving mirror of the interferometer moves. The farther the slide moves, the more periods per cycle, and the better seperation between two close periods. Our experiments are typically performed with $4 \mathrm{~cm}^{-1}$ resolution, which corresponds to about a half centimeter movement of the interferometer mirror per scan. The minimum sensitivity of the system is limited by the detector noise. The single scan noise at $4 \mathrm{~cm}^{-1}$ resolution is at about the $2 \times 10^{-3}$ level. By averaging of 1000 to 2000 scans, the noise level can be reduced to under the $1 \times 10^{-4}$ level. Reduction of noise much below this level is limited by the 15 bit resolution of the analog to digital converter at the output of the infrared detector. A single scan at $4 \mathrm{~cm}^{-1}$ resolution takes 0.5 seconds. A 2000 scan experiment then takes about 20 minutes of run time. The optical system is totally enclosed and flushed with argon to eliminate infrared absorption from atmospheric $\mathrm{H}_{2} \mathrm{O}$ and more importantly, $\mathrm{CO}_{2}$. After rapidly placing a sample in the chamber, while holding one's breath, another 20 minutes of wait was required to bring the $\mathrm{CO}_{2}$ absorption to an acceptably low level by purging with argon. The argon had the unfortunate problem of short circuiting the HeNe laser high voltage power supply in spite of podding of the lasers in insulative material. Frequent replacement of these lasers was required and thus only the centerline laser, L2, was generally tsed. 
(b)

(a)

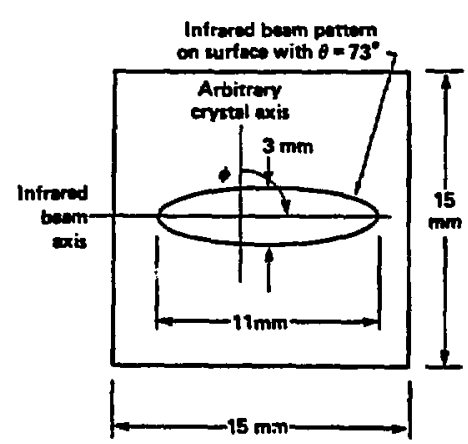

Figure V.2: Sketch (a) shows the polarization and angle of the $3 \mathrm{~mm}$ diameter infrared beam with respect to the sample. Note the internal electric field has only a small component perpendicular to the surface because of the strong refraction. Sketch (b) shows the elliptical beam pattern on the surface when the sample is at Brewster's angle $\left(\theta=73^{\circ}\right)$. The angle between the major axis of the ellipse and an arbitrary axis in the crystal plane is shown as $\phi$.

The quantum well crystal was placed in the sample chamber at the $3 \mathrm{~mm}$ diameter focus of the infrared beam. A polarizer and sample holder were placed on a translation mount and adjusted to place the sample exactly at the center of the infrared beam. Centering of the sample was particularly important since at Brewster's angle the $15 \mathrm{~mm}$ square samples only presented a $4.4 \mathrm{~mm}$ wide target to the $3 \mathrm{~mm}$ diameter infrared beam. The infrared beam was polarized horizontally by a KRS-5 window with a lithographically produced $0.4 \mu \mathrm{m}$ period metal grating on the surface. As shown in Fig. V.2, the sample was then rotated an angle $\theta$ about a vertical axis within the plane of the wafer. Because of the high index of refraction of GaAs, about 3.27 in the infrared region, Fresnel reflection off each surface of the wafer at normal incidence is about $28 \%$. These two surfaces form a Fabry-Perot cavity with a length of the wafer thickness and thus lead to very strong 
transmission oscillations as the frequency is scanned. A GaAs wafer of thickness $T$ will have a spacing between maximum cavity transmissions, $\Delta \bar{\nu}$, of $1 / 2 n_{0} T$, where

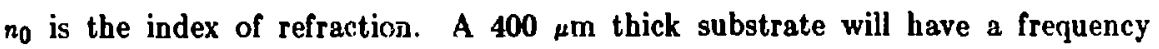
spacing between resonances of $3.8 \mathrm{~cm}^{-1}$. The scans are typically taken at $4 \mathrm{~cm}^{-1}$ resolution so as to filter out these strong oscillations in frequency. Otherwise, these Fabry-Perot oscillations, with a peak-to-peak oscillation height of about $30 \%$ of the transmission, make observation of the QWEST with a $2 \%$ absorption impossible. A further reduction in these oscillations is found by operating at Brewster's angle. Brewster's angle, $\theta_{B}$, is defined by

$$
\tan \left(\theta_{B}\right)=n_{0}
$$

and is found to be about $73^{\circ}$ for the refractive index of 3.27. At Brewster's angle our horizontally polarized infrared beam is transmitted entirely without reflection at either surface and the cavity resonance disappears. A second Fabry-Perot cavity is formed with reflection at the top surface of the epitaxial growth forming one mirror of the cavity and the interface between the eptaxial layers, consisting mostly of $\mathrm{Al}_{3} \mathrm{Ga}_{.7} \mathrm{As}$, and the $\mathrm{GaAs}$ substrate forming the other mirror of the cavity. This Fabry-Perot cavity can be shown to have a transmission peak-to-peak height of about $4 \%$. The periodicity of this resonance is found, as before, from the equation $\Delta \bar{\nu}=1 / 2 n_{0} T_{e p i}$. A typical value of the epitaxial thickness is about $3 \mu \mathrm{m}$ and using this value for $T_{\text {epi }}$, the spacing between resonaces becomes about $520 \mathrm{~cm}^{-1}$. This large a frequency spacing cannot be spectrally filtered, but because of the small amplitude of this oscillation, it can be eliminated in entirety at Brewster's angle. This resonance was extremely useful in calibrating the thickness of the overall epitaxial growth. This calibration was then used to calibrate the quantum well thickness. The procedure involved first deducing the overall epitaxial thickness 
from the Fabry-Perot cscillations with the infrared beam at normal incidence to the surface of the wafer. The ratio of the actual overall thickness to the overall design thickness is asummed constant throughout the epitaxial growth and thus the wells are multiplied by the same proportion. This assumption is not necessarily valid since the wells consist of a ciiferent material, GaAs, than the majority of the epitaxial growth, which consists mostly of $\mathrm{Al}_{.3} \mathrm{Ga}_{.7} \mathrm{As}$. However, Transmission Electron Microscope (TEM) data indicate this assumption may be valid. This TEM data is discussed in the next section.

Sample orientation at large angles such as Brewster's angle is important to see the QWEST absorption. This is because the QWEST dipole is normal to the surface and so the electric field of the infrared beam must bave as large a component as possible normal to the surface to maximize the absorption. Unfortunately the beam is strongly refracted upon entering the sample and the electric field has only a small component in the desired direction (See Fig. V.2). The angle $\theta$ must be maximized if we wish to have any coupling to the QWEST dipole. If $\theta$ is small or zero, the QWEST absorption woul! disappear. But as $\theta$ becomes very close to $90^{\circ}$, the projected area of the wafer to the infrared beam becomes very small and the 3 $\mathrm{mm}$ diameter infrared beam could intercept only a fraction of the wafer. Use of $\theta$ at Brewster's angle provides near maximum possible coupling of the infrared field to the QWEST dipole by a beam incident on the surface and yet projects a resonable cross section to the infrared beam. It also has the advantage of eliminating the Fabry-Perot resonances mentioned abo:e. Thus this angle is generally used for the QWEST absorption measureinents.

We now need to calculate the absorption strength of a quantum well sample at Brewster's angle. This calculation is complicated by the two-dimensional nature of the states and propogation of an infrared beam at an angle to these states. The 
technique to calculate this absorption is thus based on energy conservation, which allows a simple but accurate derivation frum fundamental principles. We calculate the power absorbed by the quantum wells from the infrared beam from the quantum mechanics of these states in the presence of an infrared beam. This power is then normalized by the incident beam power to deduce the absorption fraction. We begin by noting that the power absorbed per unit volume, $\mathrm{PA}$, is given by $(16)$

$$
P A=\frac{\omega E_{0}}{2} \chi^{\prime \prime}(\omega)\left|E_{z}\right|^{2}
$$

TL a power absorption per unit surface, PAS, can be found by substitution of $\rho_{0}$ for $\Delta N_{0}$ in equation (IV.84b) and using this expression for $x^{\prime \prime}(\omega)$ in equation (V.2) to obtain

$$
P A S=\frac{\omega \mu^{2} T_{2} \rho_{s}}{2 \hbar} \frac{1}{1+\left(\omega-\omega_{0}\right)^{2} T_{2}^{2}}\left|E_{z}\right|^{2}
$$

This expression was found without inclusion of the plasma interaction described in chapter $\mathrm{IV}$, sectio- $\mathrm{E}$. This interaction was found to shift the absorption maximum from $\omega_{0}$ to $\omega_{0}^{*}$, where

$$
\omega_{0}^{2}=\omega_{0}^{2}+\omega_{p}^{2}
$$

and $\omega_{p}$ is the plasma frequency defined for a quantum well by equation (IV. $\times 3$ ). The power absorption par unit area in the presence of this energy shift was given in equation (IV.82). The expression for $x$ in equation (IV.84) was derived in the rotating wave apprcximation. If we apply a similar assumption $\left(\omega_{0}^{*}+\omega \approx 2 \omega_{0}^{*}\right)$ to equation (IV.82), it becomes identical to equation (V.5) but multiplied by $\omega_{0} / \omega_{0}^{*}$, so PAS become- 


$$
P A S=\frac{\omega_{0} \omega \mu^{2} T_{2} \rho_{0}}{2 \omega_{0}^{*} \hbar} \frac{1}{1+\left(\omega-\omega_{0}\right)^{2} T_{2}^{2}}\left|E_{z}\right|^{2} .
$$

This absorption per area must be multiplied by the number of wells excited, $W$, to determine the total absorption.

The component of the electric field in the $z$ direction squared, $\left|E_{z}\right|^{2}$, must now be related to the incident power, $\boldsymbol{P}_{\mathbf{0}}$. This relation is calculated for Brewster's angle, ${ }^{\theta_{B}}$. We first note that at Brewster's angle, the power incident on the wafer surface is fully transmitted into the wafer. We also use the relation between the infrared intensity, I, and the electric field;

$$
I=\frac{n_{0}|E|^{2}}{2 \eta_{0}}
$$

where $n_{0}$ is the refractive index and $\eta_{0}$ equals $\mathbf{3 7 7}$ ohms. Using power conservation, we have a relation between $P_{0}$ and the magnitude of the electric field inside the wafer, $E_{w}$,

$$
P_{0}=\int \frac{n_{0}\left|E_{w}\right|^{2}}{2 \eta_{0}} d S_{n}
$$

where the integral is over a surface normal to the direction of propogation inside the wafer. The internal infrared beam is found to propagate at an angle $\theta_{i}$ to the normal to the surface. From Snell's law, we know $\sin \left(\theta_{i}\right)=\sin \left(\theta_{B}\right) / n_{0}=1 / \sqrt{n_{0}^{2}+1}$, where we assume the infrared beam is incident at Brewster's angle, $\theta_{B}$, and note that $\tan \left(\theta_{B}\right)=n_{0}$ by definition. The component of the internal field perpendicular to the surface, $E_{z}$, is equal to $E_{w} \sin \left(\theta_{i}\right)=E_{w} / \sqrt{n_{0}^{2}+1}$. The total absorbed power, $A P$, is equal to 


$$
\begin{aligned}
A P & =\int P A S d S \\
& =\frac{\omega_{0} \omega \mu^{2} T_{2} \rho_{0}}{2 \omega_{0}^{*} \hbar} \frac{1}{1+\left(\omega-\omega_{0}\right)^{2} T_{2}^{2}} \int\left|E_{z}\right|^{2} d S \\
& =\frac{\omega_{0} \omega \mu^{2} T_{2} \rho_{0}}{2 \omega_{0}^{*} \hbar} \frac{1}{1+\left(\omega-\omega_{0}\right)^{2} T_{2}^{2}} \int\left|E_{w}\right|^{2} /\left(n_{0}^{2}+1\right) d S
\end{aligned}
$$

where the integral of the electric field squared is over the two dimensional quantum well surface. This integral is otherwise identical to the integral of equation (V.7). The surfaces are related by the projection formula $d S_{n}=d S \cos \left(\theta_{i}\right)=d S n_{0} / \sqrt{n_{0}^{2}+1}$. Equation (V.8) now becomes

$$
\begin{aligned}
A P= & \frac{\omega_{0} \omega \mu^{2} T_{2} \rho_{0} W}{2 \omega_{0}^{*} \hbar} \frac{1}{1+\left(\omega-\omega_{0}\right)^{2} T_{2}^{2}} \frac{2 \eta_{0} P_{0}^{2}}{n_{0}^{2} \sqrt{n_{0}^{2}+1}} \\
& =\frac{2 \pi}{n_{0} \lambda_{0}} \frac{\mu^{2} T_{2} \rho_{0} W}{\epsilon \hbar} \frac{1}{1+\left(\omega-\omega_{0}\right)^{2} T_{2}^{2}} \frac{\omega_{0}^{2}}{\frac{\omega_{0}}{n_{0}} \sqrt{n_{0}^{2}+1}}
\end{aligned}
$$

where we have multiplied the absorbed power by the number of wells, $W$. If we let $\rho_{0} W$ equal the integral of the averaged three dimensional lower state density, $\Delta N_{0}$, over $z$, then it is noticed that equation (V.8) is very similar to the integral of $\gamma(\omega)$ as given in equation (IV.84). In particular, $A P=\int \gamma(\omega) d z\left(\omega_{0} / \omega_{0}^{*}\right) / n_{0} \sqrt{n_{0}^{2}+1}$. The division by the no terms is a result of the component of the field perpedicular to the surface being reduced by a factor of approximately no upon refraction at that surface. The absorption fraction, $A F$, is found by dividing $A P$ by $P_{0}$. We also use the definition of oscillator strength to find

$$
A F=\frac{\epsilon^{2}}{2 \epsilon_{0} m_{e} c} \frac{\omega_{0}}{\omega_{0}^{4}} \frac{\int \rho_{0} w}{n f^{2} \sqrt{n_{0}^{2}+1}} \frac{T_{2}}{1+\left(\omega-\omega_{0}\right)^{2} T_{2}^{2}}
$$

where the first term is seen to contain only fundamental constants, the second term, which is a measure of the effect of the plasma shift, is nearly unity, the third term 
contains the product of the oscillator strength and the number of oscillators, divided by the refractive power of the wafer approximately to the cube, all of which are material parameters, and the iast term is simply a lineshape factor. Because the lineshape will be hard to predict and we wish to obtain a relation for the oscillator strength independent of lineshape, we integrate the absorption fraction over the lineshape to obtain an integrated absorption fraction, $I A F$, given by

$$
\begin{aligned}
I A F & \equiv \int A F(\omega) d(\hbar \omega) \\
& =\frac{e^{2} h}{4 c_{0} m_{e} c}-\frac{\omega_{0}}{\omega_{0}^{t}} \frac{\int \rho_{0} W}{n_{0}^{2} \sqrt{n_{0}^{2}+1}} .
\end{aligned}
$$

This expression is evaluated using $W=50$ and $\rho_{1}=4 \times 10^{11} \mathrm{~cm}^{-2}$, and $\omega_{0} / \omega_{0}^{*}=$ 0.975 to obtain $I A F=0.062 \mathrm{fmeV}$. The infrared spectrometer uses units of Absorbance to measure absorption. The Absorbance (Abs) is defined by $\mathrm{Abs}=-\log _{10}$ (Transmission). If the transmission is close to unity then Abs=Absorption/ln (10) where Absorption=1Transmission. For instance, a $1 \%$ absorption will give an absorbance of $0.01 / \ln (10)=4.34$ $\mathrm{mAbs}$, where $\mathrm{mAbs}=10^{-3} \mathrm{Abs}$. The frequency, $D$, is measured in units of inverse centimeters and is related to the photon energy, $E$, in $\mathrm{eV}$ by $E=h c \bar{\nu}=$ $1.24 \times 10^{-4} \bar{i}$. For example, a $1000 \mathrm{~cm}^{-1}$ frequency corresponds to an energy of $124 \mathrm{meV}$. The integrated linestrength becomes, in units of the spectrometer, $I A F=0.218 \mathrm{f} \mathrm{Abs-cm}^{-1}$. This expression is used to measure the oscillator strength of each of the quantum wells. 


\section{B. (AIGa)As QW Crystal Development}

The quantum well crystal growth evolved over the course of several years of development. The type of structures made and the manner in which they were made were both critical to the proper observation of the QWEST. In fact, it was not until a few months ago that this growth procedure became sufficiently refined to see any significant effect. However, once the proper technique for growth was found, the QWEST appeared in full strength with near perfect agreement with theory.

The crystal growth was performed with the aid of Steve Eglash, a Stanford graduate student, using the Molecular Beam Epitaxy (MBE) apparatus at HewlettPackard. Hewlett-Packard generously agreed to cooperate by providing these samples. One of the problems was that Hewlett-Packard had not, previous to my request, grown quantum well crystals. However, they had grown heterojunctions of (AIGa)As on GaAs. These surfaces are capable of quantizing an electron in the direction perpendicular to the surface and were used to make high mobility transistors. These heterojunctions were well characterized and it was felt that quantum wells, which merely required another hetrojunction on the other side, would be a simple step beyond the known crystal growth procedure. This turned out not to be the case. First, the previous crystal growing procedures had some errors in many of the growth parameters. Second, it is now known that it is much easier to grow (AlGa)As on GaAs and obtain a good interface than it is to grow GaAs on (AlGa)As. The reasons are still not well understood, but appears to be related to how impurities are incorporated into the two materials. $[35,26]$

The first samples grown, in May of 1982, were a set of three crystals, with one well each of a single quantum well of GaAs with $\mathrm{Al}_{.3} \mathrm{Ga}$. As barriers. One well was grown in each sample of 0,80 , and $100 \AA$ thickness, respectively. The $\mathrm{Al}_{3 .} \mathrm{Ga}_{.7}$ " s barriers were $100 \AA$ thick. The doping was in the $\mathrm{GaAs}$ well region with a $\mathrm{Si}$ 
donor density of $4 \times 10^{17} \mathrm{~cm}^{-3}$ The absorption spectra for these samples had little absorption at the higher energies and quickly went to infinite absorption for energies around $1200 \mathrm{~cm}^{-1}$ (or $\sim 150 \mathrm{meV}$ ) and below. It was quickly realized that the substates were not insulating. The observed absorption spectra matched perfectly with that expected from a Drude model with a doping density of $5 \times 10^{18} \mathrm{~cm}^{-3}$, a scattering time of 0.2 picoseconds (from the mobility) and a substrate thickness of $0.34 \mathrm{~mm}$. Hewlett-Packard confirmed these substrate properties.

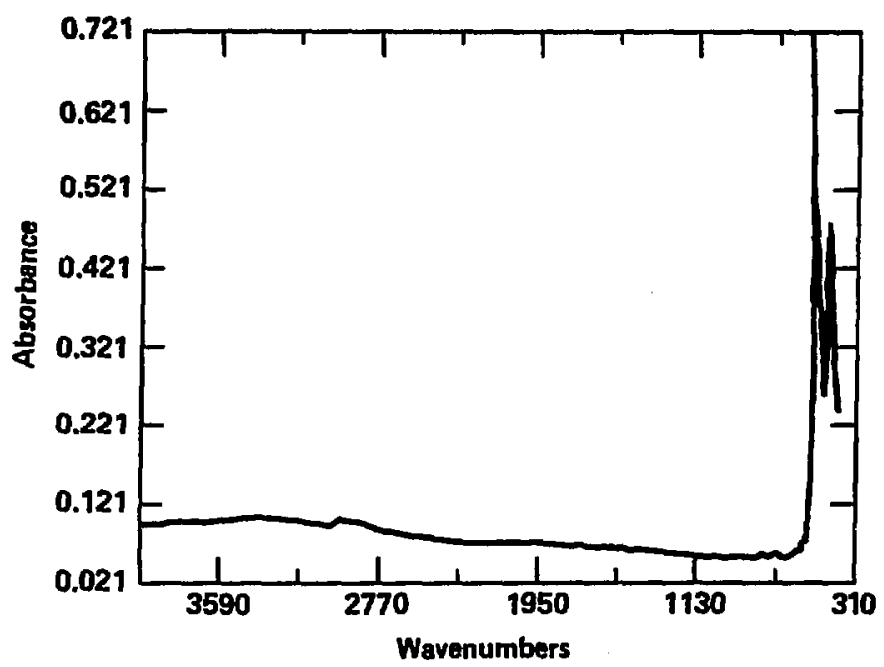

Figure V.3: An example of a typical infrared absorption spectra of a GaAs substrate with epitaxial growth of (AlGa)As quantum wells. This sample, A-516, failed to show any QWEST resonances, but exhibites the two small phonon abosrption peaks at 709 and $770 \mathrm{~cm}^{-1}$ seen on all of the later samples. Note that (AlGa)As materials have very little absorption in a broad region of the infrared from 2 to 20 micron wavelengths. (Absorbance $=-\log _{10}($ tranemission $)$ ) 
The next set of three samples were grown as before but on insulating substrates. The spectra is flat except for a strong absorption band which occurs for wavenumbers below about $500 \mathrm{~cm}^{-1}$ from optical phonon absorption. [4] On these samples is also observed a small peak at $770 \mathrm{~cm}^{-1}(95.5 \mathrm{meV})$ with a height of about $5 \mathrm{mAbs}$ and a width of $5 \mathrm{meV}$. This peak was the same on all samples and independent of polarization of the infrared beam. No other absorptions were observable. A typical infrared spectra of a GaAs substrate with epitaxial growth is shown in Fig. V.3, which is the spectra of a later sample, A-516, which shows an absorption at both $770 \mathrm{~cm}^{-1}$ and $700 \mathrm{~cm}^{-1}$.

During the months of October and November, 1982, several different samples were investigated to better understand the infrared spectra of GaAs and its epitaxial growth. Four samples were provided by Steve Eglash of Hewlett-Packard as exemplary of various types of substrates and epitaxial growth. The first of these samples, G-718, is a GaAs insulating substrate doped with $\mathrm{Cr}$ and $\mathrm{Te}$, which have midgap bound energy states, to insure the Fermi level is pinned at midgap. This substrate is the most commonly used form of insulating substrates. A second pure GaAs substrate, without any doping at all, G-739, was also provided. Two samples with epitaxial growth were also examined. Both samples had $4.5 \mu \mathrm{m}$ of $\mathrm{Al}_{\mathrm{X}} \mathrm{Ga}_{1-\mathrm{x}} \mathrm{As}$ grown eptiaxially by Hewlett-Packard's MBE machine. The first $0.8 \mu \mathrm{m}$ of growth was undoped and the latter $3.6 \mu \mathrm{m}$ was grown with about $8 \times 10^{17} \mathrm{Si}$ donors. For one of these samples, A-388, the Al fraction, $x$, is 0.18. For the other sample, A399, the Al fraction, $x$, is 0.28 . The samples G-739 and G-718 exhibit no narrow absorption peaks above the $500 \mathrm{~cm}^{-1}$ phonon absorption edge and have a noise level of $0.6 \mathrm{mAbs}(=0.014 \%$ absorption). In particular, neither of these substrate materials exhibit either the 700 or $770 \mathrm{~cm}^{-1}$ resonances. Also ssen are shallow maxima, slowly varying over 100's of wavenumbers (10's of meV'), with amplitudes 
of about $2 \mathrm{mAbs}$. These are common throughout the spectrum and are believed to be noise. Both samples A-388 and A-399 have similar spectra but with an absorption observable at $70 \mathrm{~cm}^{-1}$. This resonance has a height of $5.3 \mathrm{mAbs}$ for sample A-388 and 11.8 for sample A-399. The $770 \mathrm{~cm}^{-1}$ resonance is not seen on any of these samples. However, for all samples measured after this set, the $\mathbf{7 7 0}$ $\mathrm{cm}^{-1}$ resonance is observed. On a later set of samples, we fird that the substrate, F620ES, has an absorption at $770 \mathrm{~cm}^{-1}$ but not at $709 \mathrm{~cm}^{-1}$. The samples B-133 and B-134, with a few microns of epitaxial growth on the same substrate material as F620ES, subsequently exhibit an additional line resonance at $700 \mathrm{~cm}^{-1}$. The absorption peak at $770 \mathrm{~cm}^{-1}$ has been previously reported to be a third order optical phonon absorption in GaAs/(2). This third harmonic absorption is probably enhanced by various crystal defects or impurities. This peak was not seen on any of the older samples, including those with epitaxial growth. However, it is seen on all of the newer samples, including those with no epitaxial growth. (HewlettPackard changed their crystal suppliers about the time of the change.) In addition to this resonance at $770 \mathrm{~cm}^{-1}$, another very similar resonance appears at $709 \mathrm{~cm}^{-1}$ $(87.9 \mathrm{meV})$ only for those samples with over a micron of epitaxial growth. Tests on wafers both before and after growth indicate that the $709 \mathrm{~cm}^{-1}$ absorption is generated by the epitaxial growth. The $700 \mathrm{~cm}^{-1}$ absorption is suspected to be the same phenomena as the $770 \mathrm{~cm}^{-1}$ absorption, only occuring in (AIGa)As instead of GaAs. Note the energy of this $709 \mathrm{~cm}^{-1}$ line is observed at exactly the same frequency for both $\mathrm{Al}_{18} \mathrm{Ga}_{.82} \mathrm{As}$ and $\mathrm{Al}_{.28} \mathrm{Ga}_{.72} \mathrm{As}$ epitaxial growths, but with about twice as large an absorption for the latter. Thus this absorption is more related to the presence of $\mathrm{Al}$ than the properties of (AlGa)As. Other than these phonon resonances, no other peak which could be confused with a QWEST was observed on any sample. 
The absorption experiments with the above set of samples allowed a calibration of the minimum noise of the spectrometer. This is found to be equivilent to about $10^{-4}$ absorption strength. But depending on such factors as the $\mathrm{CO}_{2}$ content of the spectrometer and the surface quality of the wafer, the noise of transmission through a GaAs wafer is variable but often several times $10^{-4}$. A single quantum well doped in the well region with a $10 \mathrm{meV}$ linewidth is calculated to have ove: a $10^{-4}$ absorption. However, caution and the lack of observation of a QWEST at this level indicated further samples should be grown with $\mathbf{5 0}$ wells to increase the absorption signal. The doping was also changed from the well region to the barrier region. The electrons would then diffuse into the well and populate the lowest quantum well. Two advantages are seen for doping in the barrier regica. The first is that the lower energy quantum well state is fully populated by the donor electrons and this population density is independent of temperature. As the lower quantum well energy tends to be more than $50 \mathrm{meV}$ above the GaAs band edge, which is the location of the Si donor energy with doping in the well region, only $10 \%$ of the donor electrons populate the lower state at room temperature and much less at lower temperatures. In contrast, the $\mathrm{Si}$ donors in the $\mathrm{Al}_{.3} \mathrm{Ga}_{.7} \mathrm{As}$ barrier have a donor energy only a few meV below the barrier band edge, but 100's of $\mathrm{meV}$ above the GaAs quantum well states, thus the electrons filly ionize into the lowest quantum well states for all temperatures. The electrostatic energy between fully ionized donors and free electrons in the well for a surface density of $1 \times 10^{11} \mathrm{~cm}^{-2}$ is easily shown to be only about $30 \mathrm{meV}$ for $100 \AA$ wells and doping regions. Since the barrier is about $260 \mathrm{meV}$ high, the relatively weak electrostatic forces allow full donor ionization into the well region. The effect of the electrostatic forces on the quantum well energies was already covered in chapter $I V$, section $E$. This change increases the room temperature population of the quantum wells, and therefore the absorption 
strength, by a factor of about 10 for the same doping density. So this increase, in combination with the growth of 50 wells, increases the absorption strength by $\approx$ factor of about 500 to about $5 \times 10^{-2}$ or $5 \%$. This absorption strength is very clearly observable witi: the FTIR spectrometer with a signal to noise ratio of over 30. A second advantage to doping in the barrier region is the isolation of the ionized donors from the free electrons, which would undoubtedly belp to conserve matching of the transverse $\mathbf{k}$ vector (because of reduced impurity scattering), essential for a QWEST linewidth sufficiently small to see the absorption and for the transition to be usefal once seen.

\begin{tabular}{|c|c|c|c|c|}
\hline La & & Thickness & Material & Doping \\
\hline & ation cap & $500 \AA$ & GaAs & Undoped \\
\hline & er region & $1.0 \mu$ & $\mathrm{Al}_{3} \mathrm{Ga},{ }_{7} \mathrm{As}$ & Undoped \\
\hline & Barrier & $50 \AA$ & $\mathrm{Al}_{3} \mathrm{Ga}, 7 \mathrm{As}$ & $N_{D}=4 \times 10^{17} / \mathrm{cm}^{3}$ \\
\hline 1 & Well & $L_{w}$ & GaAs & Undoped \\
\hline & Barrier & $100 \AA$ & $\mathrm{Al}_{.3} \mathrm{Ga}_{3} \mathrm{As}$ & $N_{D}=4 \times 10^{17} / \mathrm{cm}^{3}$ \\
\hline & Well & $L_{w}$ & GaAs & "Undoped \\
\hline & Barrier & $100 \AA$ & $\mathrm{Al}_{3} \mathrm{Ga}_{2} \mathrm{As}$ & $N_{D}=4 \times 10^{17} / \mathrm{cm}^{3}$ \\
\hline ! & Well & $L_{w}$ & GaAs & Undoped \\
\hline & Barrier & $100 \mathrm{~A}$ & $\mathrm{Al}_{3} \mathrm{Ga}_{7} \mathrm{As}$ & $N_{D}=4 \times 10^{17} / \mathrm{cm}^{3}$ \\
\hline & Well & $L_{w}$ & GaAs & \\
\hline & Barrier & $50 \AA$ & $\mathrm{Al}_{3} \mathrm{Ga}_{7} \mathrm{As}$ & $N_{D}=4 \times 10^{17} / \mathrm{cm}^{3}$ \\
\hline & r region & $1.0 \mu$ & $\mathrm{Al}_{3} \mathrm{Ga}_{3}$ As & Undoped \\
\hline
\end{tabular}

GaAs substrate undoped

Figure V.4: Layer structure of the epitaxial quantum well growth for the earlier samples. 

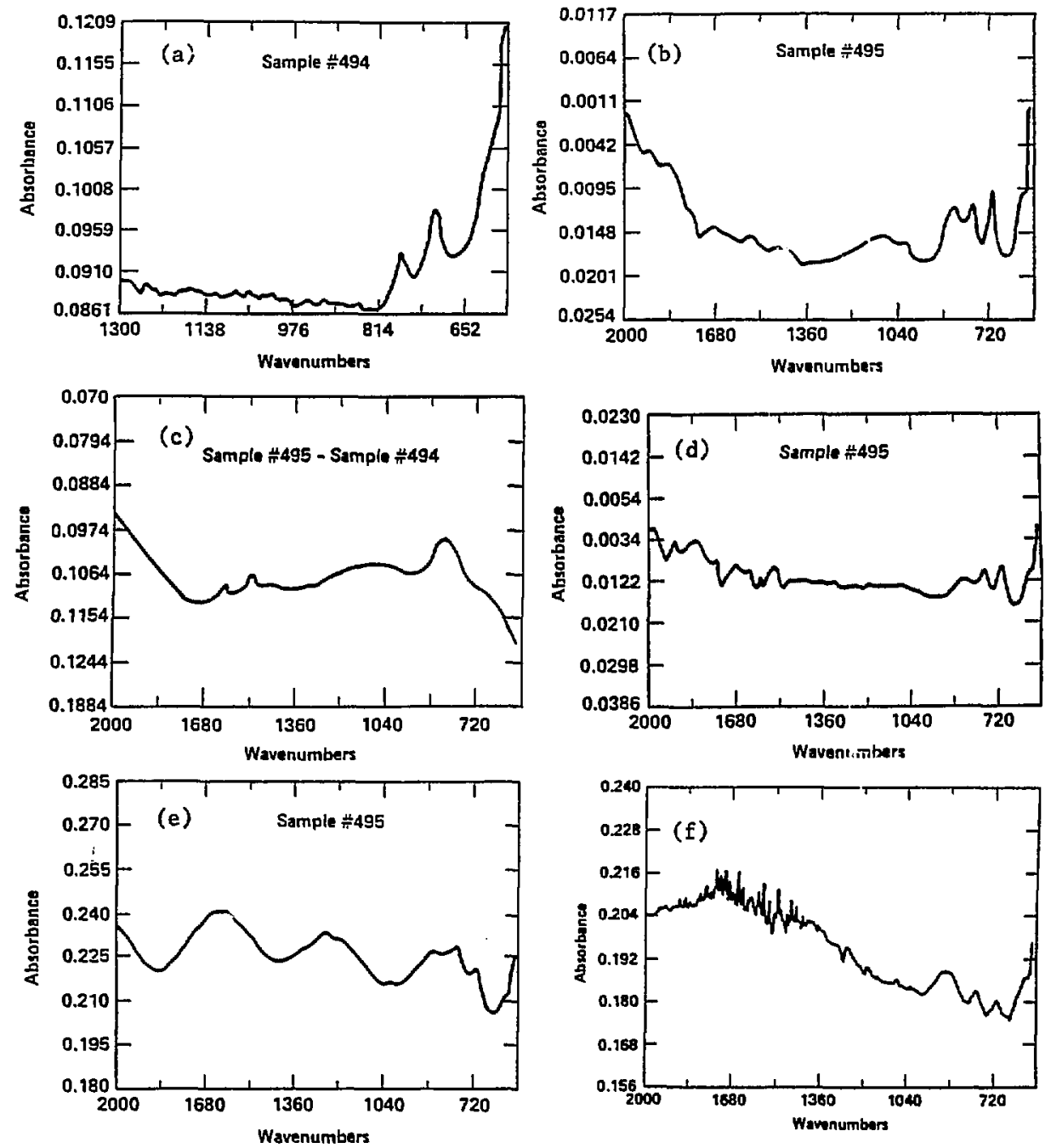

Figure V.5: Infrared absorption spectra of quantum well samples at $\theta=73^{\circ}$ (except where indicated) and at room temperature. a.) Sample A-494, b.) Sample A-495, c.) Difference spectra of samples A-495 and A-484, d.) Sample A-495, two days after spectra b.) was taken (note the differences), e.) Sample A-495 at normal incidence $(\theta=0)$, f.) Sample A-495 two months after spectra $b$. above (note the energy shift of one peak). 
Samples A-494 and A-495 were then grown, as shown in Fig. V.4, on January 3, 1983. The two samples, A-494 and A-495, were grown with 50 wells and were specified to have well thicknesses, $L_{w}$, of $80 \AA$ and $65 \AA$, respectively. The epitaxial growth was performed with a constant substrate temperature of $580^{\circ} \mathrm{C}$ for both the (AlGa)As and GaAs regions. No sample was grown with $L_{\omega}$ equal to zero since the infrared spectra of the epitaxial growth had become sufficiently well understood to not requito this sample. Furthermore the epitaxial growth was becoming sufficiently thick, about $3 \mu \mathrm{m}$, that it took 4 hours to grow a single sample, and a iull day at a time seemed near the limits of Hewlett-Packard's generosity. This meant two samples. These samples were polished on the back side and probed within 2 days of their growth. Unexpectedly, this rapid response was critical to the first observation of the QWEST. The spectia of these samples are shown in Fig. V.5. As indicated above and in equation (V.11), a $10 \mathrm{meV}$ linewidth transition is expected to have a $30 \mathrm{mAbs}$ absorption strength. The spectrum of sample A-494 is seen in Fig. V.5a to have the commonly observed phonon peaks at 709 and $770 \mathrm{~cm}^{-1}$ but no other transitions indicative of a QWEST. Sample A-494 was specified to have $80 \AA$ thick wells and thus should have a QWEST frequency of about $1000 \mathrm{~cm}^{-i}$. The spectrum of sample A-495 taken on January 5, 1983, is illustrated in Fig. V.5b. This sample with $65 \AA$ thick wells should have a resonance at about $1220 \mathrm{~cm}^{-1}$. No resonance is seen at this energy, but it is noticed that a new absorption peak, never observed before on any of the previously measured samples, is seen at $835 \mathrm{~cm}^{-1}$ (or 104 $\mathrm{meV}$ ). This new resonance is made clear by the subtraction of the two quantum well samples in Fig. V.5c. This transition has a linewidíh of $10 \mathrm{meV}$ and a peak height of about $8 \mathrm{mAbs}$. This gives a linestrength about a factor of 3 below that predicted. Two days later, on January 7, 1983, the spectrum of Fig. V.5d was taken. The anomalous resonance is seen to have shifted about $0.3 \mathrm{meV}$, broadened 
slightly, and most significantly, decreased in peak height by about a factor of two. A few days later it disappeared altogether. The spestrum of this sample at normal incidence, taken on January 7,1983 , is shown in Fig. V.5e. It is noticed that the absorption peak height has decreased by about a factor of 2 or 3 but does not go to zero, as it should do if it were a QWEST. Yet another anomaly appeared two months later after sample $A-495$ had been heated to $450^{\circ} \mathrm{C}$. for approximately 45 minutes (in an attempt to diffuse in some In) and re-examined. The QWEST resonance is seen in Fig. IV.5f to reappear, but shifted by an energy of $6.6 \mathrm{meV}$. This spectra will be discussed later.

The above observations of samples A-494 and A-495 in early January, 1883, brought up many questions. One problem was that if this transition was a QWEST, then why is its energy so far from prediction? The answer is that the wells were not the specified thickness. This problem had already been indicated in samples A-388 and A-399. The infrared spectrum of these samples at normal incidence allowed determination of the epitaxial thickness from the Fabry-Perot resonance of this thin optical cavity. Using an index of refraction of 3.2 for the (AlGa)As epitaxial growth, $[43]$ the resonant peak spacing indicated the epitaxial thickness of the two samples, A-388 and A-399, were 5.7 and $6.6 \mu \mathrm{m}$, respectively. This is contrasted with the specified thickness of $4.5 \mu \mathrm{m}$ for both the samples. A SEM scan of the edge of the wafer indicated the thickness of the two samples were much larger than $4.5 \mu \mathrm{m}$, but the SEM calibration was not correct to better than $10 \%$ and could not give an independent accurate measurement. These SEM photos did indicate, however, that the two samples were not of the same thickness and that A-399 has an epitaxial thickness about 15\% larger than that of A-388. HewlettPackard revealed that their method for calibration the epitaxial thickness relied on an optical micruscope with about the same = Jlution as the $\mu \mathrm{m}$ growths, so their 
accuracy was in question. But the accuracy of the infrared cavity measurements had not been truly verified. The distanice between the cavity resonance peaks could usually be determined with an accuracy of about $3 \%$. But the accuracy of the index of refraction was in doubt. The refractive index of GaAs at $10 \mu \mathrm{m}$ wavelengths was found to have numbers as different as 3.095 and 3.309. $(44)$ Our calculations indicated that $\mathrm{GaAs}$ should $\mathrm{b}^{\mathrm{m}} \mathrm{e}$ an index of refraction of 3.30 at 10 $\mu \mathrm{m}$ wavelengths. $[43]$ An attempt was made to determine the refractive index by increasing the resolution of the spectrometer sufficiently to see the interference resonances of the entire wafer. This measurement gave the results of 3.13 and 3.25 . The limitation of this determination of index was the difficulty in measuring the thickness of a 0.011 inch thick wafer with an accuracy bette: than 0.0003 inches with the tools immediately available (one problem is that the samples appear to have a wedge and other spatial nonuniformities at this scale). An analysis of the literature gave $a$ strong indication that the index of refraction was in the range of 3.27 to 3.30 , within $1 \%$ of the theoretical derivation. [43] The theoretical model[43] alsc predicted an index of refraction of 3.16 for $\mathrm{Al}_{3} \mathrm{Ga}_{.7} \mathrm{As}$. These became the two values used for GaAs and the epitaxial growth, respectively, and are suspected to be accurate within 1\%. The accuracy of the epitaxial growth then became that of determining the resonance period in the proper frequency region. This measurement is usually accurate to within about $3 \%$ and thu limited the ovarall accuracy of the epitaxial thickness measurement to a similar amount. But this accuracy is quite adequate to conclude the epitaxial growths were significantly larger than predicted. But the variation in the thickness of samples A-388 and A-399 was a mystery, since even if the calibration of the growth rate uas wrong, it should be repeatable. One possiblity is that the additional $10 \% \mathrm{Al}$ added to the molecular beam could have increased the growth of sample A-399 by $20 \%$ over that of sample A-388. Another 
possiblility is that the samples simply came from different parts of the 2 inch wafer. Evidence will be shown later that variations in thickness ver the wafer can be over $10 \%$. In light of these uncertainties, Hewlett-Packard decided to reiy on their previous calibrations when growing samples A-494 and A-495.

The period of the oscillations in Fig. V.5e was found to be $390 \mathrm{~cm}^{-1}$. Using $n_{0}=3.16, T=1 / 2 n_{0} \Delta \bar{\nu}=4.06 \mu \mathrm{m}$. But the sum of the layers in Fig. V.A add up to $2.875 \mu \mathrm{m}$ using $L_{w}=65 \AA$. If we assume the GaAs well has zrown by the same ratio as the overall epitaxial thickness, then we find the well thickness is actually 1.411 times larger than $65 \AA$, or about $92 \AA$. A similar expansion exists for sample A484. This increase in the well width gives a theoretical resonance frequency for the QWES' 1 ' of $911 \mathrm{~cm}^{-1}$ for sample A-495, now in the neighborhood of the anomalous resonance peak. The resonance energy of sample A-494 is similarly increased and predicted to be at about $720 \mathrm{~cm}^{-1}$. Although this resonance energy should be observable, it could be lost in the phonon spectra. The increased well thickness, if it occurs, offers some explainations to the observations to samples A-494 and A495. But it was still not absolutely certain that the infrared technique for epitaxial thickness was valid. Even should the overall thickness prove to be as accurate as expected, the well is grown with a different material, GaAs, froin the bulk of the epitaxial growth, which was $\mathrm{Al}_{3} \mathrm{Ga}_{7} \mathrm{As}$, and the excess growth need nest have the same ratio in the two. Fortlinately, both problems were answere: cc 'usively in a highly sophisticated probe.

During this time period, Fernando Ponce of Hewlett-Packard was developing a highly sophisticated Transmission Electron Microscope. He asked Steve Eglash if he had axy inturesting samples to observe and Steve happened to give Fernando a piece of sample A-495 to work with. In addition to resolving the well and barrier regions, Fernando found that by orienting the surfaces in the [110] direction and 
thinning the samples to about 50 atoms thick, he could resolve individual Ga-As atom pairs in the quantum well layers (see Figs. V.6 and V.7). From this photo of the structure, several conclusions can be made about the quantum well layers. First, a direct ratio of the barrier width to the well width shows that indeed the 65 to 100 ratio is maintained in the growth. In addition an absolute calibration of the well width can be found by counting the number of GaAs unit cells in the height of the well. This number, depending on which plane the count start and stops, appears to be about $33 \pm 1$ half-unit-cell layers. The lattice spacing of $\mathrm{GaAs}$ is known from X-ray diffaction measurements to be/45] $5.65315 \AA$, making a 33 layers of atoms equal to about $93.277 \AA$. This dimension is in good agreement with that based on the epitaxial cavity resonances and gave us confidence in this technique for calibration of the epitaxial thickness. (This is probably one of the more unusual methods used to calibrate the index of refraction of a material.) 


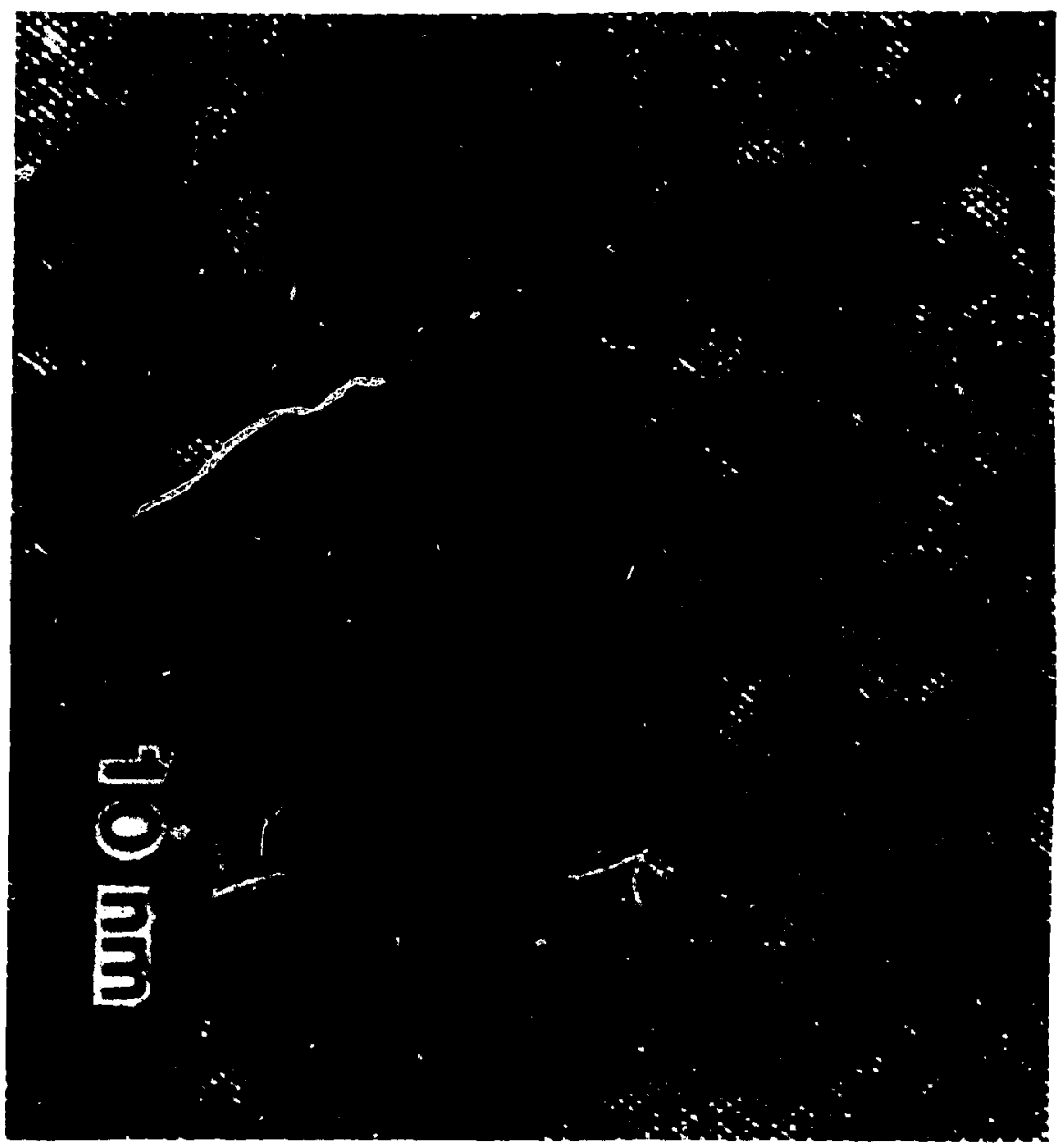

Figure V.6: A very high resolution TEM photograph of sample A-495 in the [011] direction, showing atomic resolution of the well and barrier regions and indicating a high quality interface has been created. The $10 \mathrm{~nm}$ marker is slightly incorrect, the thickness of the black well region is actually about 33 half cells of the FCC lattice, or $\mathbf{9 3 . 2 7 7} \AA$. (Photograph courtesy of F. Ponce at Hewlett-Packard Laboratories) 


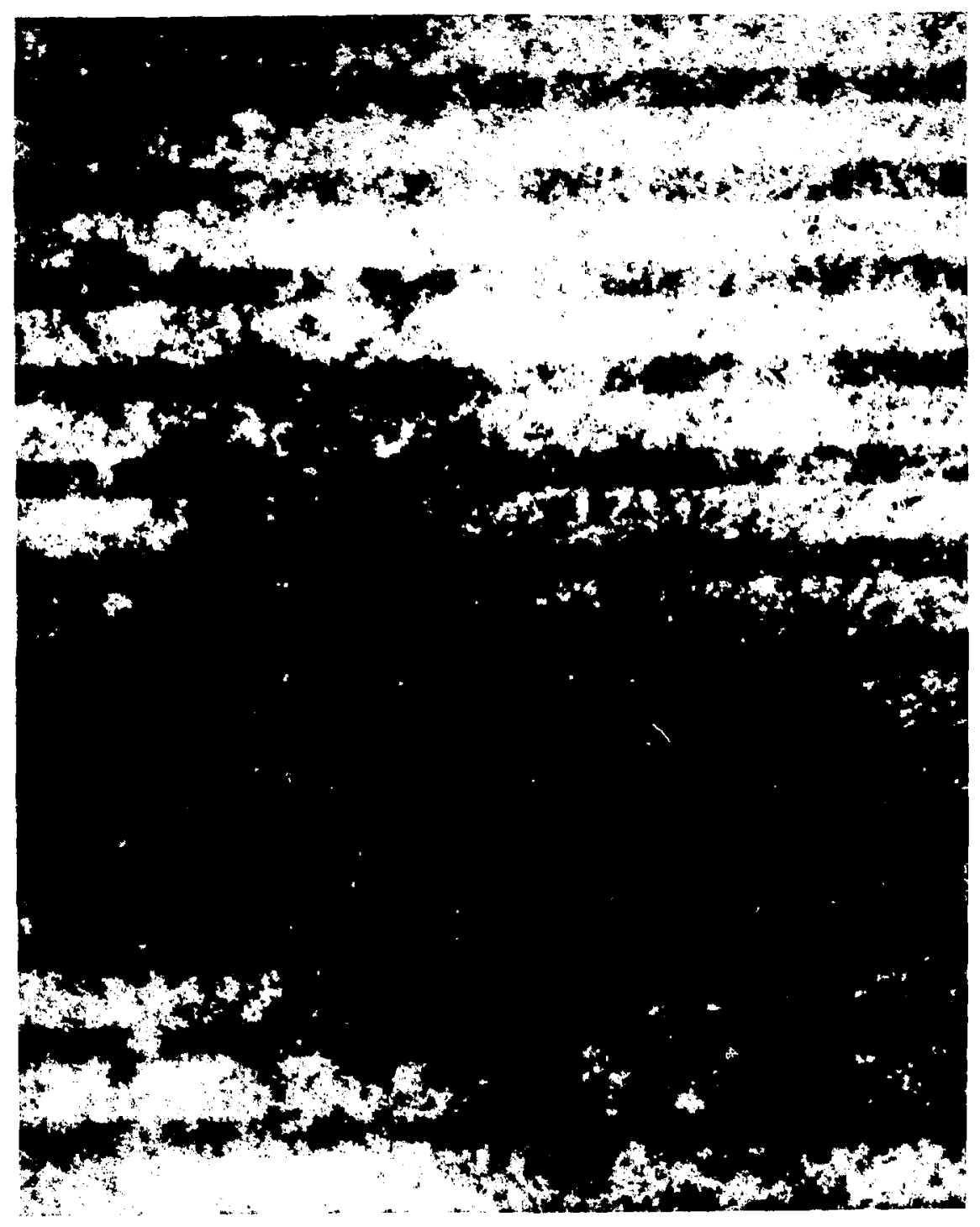

Figure V.7: A lower magnification of Fig. V.6 showing several of the 50 quantum wells grown for sample A-495. 
The observation of samples A-494 and A-495 could then have a simple explanation. The resonances of both samples were shifted into the infrared because the wells were excessively large. One resonance, of sample A-405 was observed, but that of sample A-494 was lost in the infrared or else disappeared in a similar fashion to the resonance of A-495, only on a faster time scale. A sample of the same layer structure as these two, but with a smaller well, was grown in an effort to see a second resonance with a higher energy. This sample, A-516, was grown on January 19,1983 and observed on the same day as its growth. The well width was grown to be $7 / 9$ of the one in sample $A-495$, or about $70 \AA$. Unfortunately, this sample showed no additional resonances other than those commonly observed at 770 and $709 \mathrm{~cm}^{-1}$. This sample was exhibited in Fig. V.3.

The lack of observation of a QWEST on these samples is in direct conflict with theory. The theory of how a transition between states occurs was examined further in an attempt to find effects which might destroy or counteract such a transition. None were found. The samples were then examined further to see how they might. have failed. One of the more suggestive failure modes is the leakage of the electrons out of the lowest quantum well states into lower energy bound states. These bound states could either arise from impurities in the system or interface states someho: being created. Hewlett-Packard had years of experience with the crystal growth and had reduced the impurity levels to less than $10^{15} \mathrm{~cm}^{-3}$ in the bulk structures they had grown. Our quantum wells are doped with $4 \times 10^{17} \mathrm{~cm}^{-3}$ electrons so that it was hard to see the electrons going into bulk impurity states. The other possibility was interface states. These interface states could arise either from impurities being concentrated on the interface or else from poor interfaces having large numbers of dislocated bonds or other surface states which could destroy the effect. A test of the lower state population can be made simply by measurement of the Hall resistance of 
the sample. Unfortunately, connecting to these quantum wells in our thick samples was difficult. Normally the test is performed by diffusing In into the sample to make ohmic contact. But with our samples so thick this connection could not be assured. When a sample with such a contact was measured, the readings would tend to have very high resistances and give unphysical results. Electrical experiments performed at Hewlett-Packard indicated that sample A-495 had about one half of the expected doping and the other two samples gave ambiguous results. Steve Eglash claimed that the ambiguous results are consistent with no electrons in the sample. I initially attempted to test sample $\mathrm{A}-\mathbf{4 9 5}$ by diffusing In into the sample by placing four dots of In on the edges of the wafer and heating the sample in an oven for 30 minutes at $400^{\circ} \mathrm{C}$. Because the In did not appear to be melted (later found to be an illusion created by an exterior crust formation), the sample was heated for an additional 45 minutes at $450^{\circ} \mathrm{C}$. This test of conductivity exhibited high resistances and ambiguous results. The problems with these measurements is believed to arise from the difficulty of In diffusion through many microns of semiconductor and making connection with 50 wells simultaneously, all srparated by insulating material. I then tried a new technique to make contact on a seperate piece of sample A-495 by performing a selective etch to create holes with the edge of the hole slanted outwards to expose all $\mathbf{5 0}$ well regions. A Au-Ge alloy ohmic contact was then evaporated into the hole. Four holes were made in a row and the conductivity was measured with a four point probe. Assuming a mobility of $5,000 \mathrm{~cm}^{2} / V$-sec, an electron density of about half that of the doping level was found.

The piece of sample A-495 which had been heated to $450^{\circ} \mathrm{C}$. in an attempt to diffuse In into the edges was placed back into the spectrometer. The spectrum of Fig. V.5f was observed. The resonance is now seen to have returned, but shifted $t$, in frequency of $888 \mathrm{~cm}^{-1}$ or an increase in energy of $6.6 \mathrm{meV}$ ! No explanation call 
be found for this behavior. However, as will be shown later, all of the observations exhibit shifts over long periods of time in their resonant energy and line shape.

In view of the results of sample A-495, sample A-516 was heated in an oven for 20 minutes at $450^{\circ} \mathrm{C}$. The spectrum was measured within 30 minutes of removal from the oven, but exhibited no change over its previous spectrum, with no observations of new transitions.

At this time, Hewlett-Packard's MBE machine was taken out of service for about. 9 months for improvements and cleaning. I used this time to search the literature for the procedures and problems encountered by other groups in their MBE epitaxial growth. Fortunately, several discoveries of importance to our growth were emerging. First, several reports indicated the surfaces of a quantum well are not equally high quality on both sides of the well. $(35,36,40) \mathrm{A}$ conclusive demonstation of this is reported by Morkoç et. al. in Ref. 46. They c impare the mobilty of heterostructures with (AlGa)As grown on GaAs, called a "normal structure", with that of GaAs grown on (AlGa)As, called an "inverted structure", as a function of growth temperature of the substrate. The $78 \mathrm{~K}$ mobility for the normal structure is found[48] to be about $85,000 \mathrm{~cm}^{2} / \mathrm{V}$-s for growth temperatures from 600 to $700^{\circ} \mathrm{C}$. Above this growl l, temperature, the $78 \mathrm{~K}$ mobility dreps rapidly to $10,000 \mathrm{~cm}^{2} / \mathrm{V}$-s at $750^{\circ} \mathrm{C}$. Thr inverted structure has an entirely different behavior. The inverted structure hw' a very low $78 \mathrm{~K}$ mobility of about $1000 \mathrm{~cm}^{2} / \mathrm{V}$-s for growth temperatures in the region of 600 to $660^{\circ} \mathrm{C}$. The $78 \mathrm{~K}$ mobility then rapidly rises to a maximum of 8000 $\mathrm{cm}^{2} / \mathrm{V}$-s at a growth temperature of $700^{\circ} \mathrm{C}$, after which it quickly drops again. The mobility is an indication of the interface quality, so these results suggest that the one side of the quantum well is a poor interface and its quality is eritically dependent on growth temperature. The excition linewidth is also an indicator of interface quality as interface variations will vary the energy of the quantum well 
states. This linewidth is given as function of energy ${ }^{[31]}$ in Fig. IV.6. This linewidth is much more sensitive to larger ( $\sim 100 \AA$ or greater) lateral variations in well thickness. $[17]$ But both these measures of interface quality find the best interfaces occur at a substrate growth temperature of 680 to $700^{\circ} \mathrm{C}$.

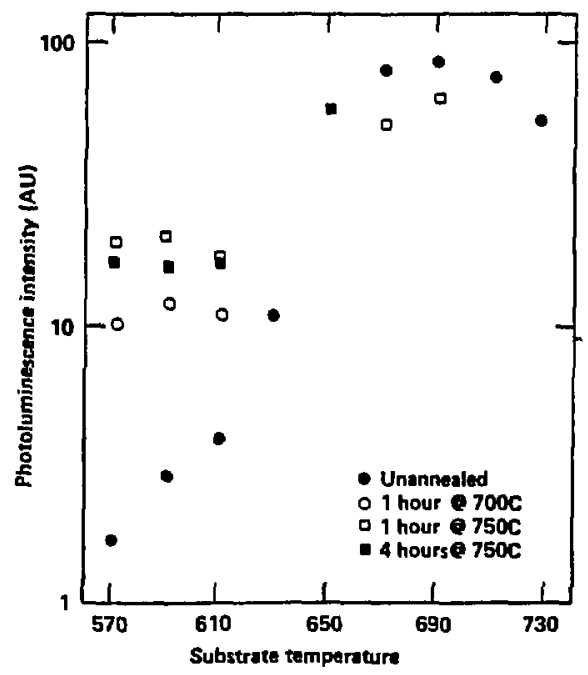

Figure V.8: A plot of the photoluminescence intensity of various quantun well samples as a function of the substrate growth temperature. Annealing of the samples, at temperatures up to $750 \mathrm{C}$ for up to 4 hours, was found to increase the photoluminescence intensity as shown (from Ref. [31]).

The peak photoluminescence intensity of GaAs quantum wells with $200 \AA$ thick wells and $200 \AA$ thick barriers is also measured at $1.6 \mathrm{~K}$ as a function of substrate growth temperature ${ }^{[31]}$ with the results shown in Fig. V.8. The photoluminescence intensity is seen to improve dramatically as the growth temperature is increased from 610 to $650^{\circ} \mathrm{C}$. The photoluminescence intensity is felt to be an indication of the purity of the quantum wells. A low photolumicescence intensity is thought to be caused by the presence of electron traps with energies in the band gap which allow the conduction band electron to nonradiatively decay to the lower valence 
band. If these traps exist, then they could also capture electrons which have been doped into the well region. This could explain the disappearance of our quantum well electrons.

The problems with the interface structure are thought to be related to the way in which impurities such as carbon are incorporated into the epitaxial layers. A common view/35,30] is that the impurities do not easily incorporate themselves into the (AlGa)As material, but instead lie on the surface of the growth region until a GaAs layer is grown, at which point these impurities are deposited into the crystal. A test of this explanation is performed by growing a series of $10 \AA \mathrm{GaAs}$ welli: in the (AlGa)As barrier immediately before growing a test quantum well of larger thickness. These tests show an increase in photoluminescence efficiency by a factor of $7[35]$ and $160 .(30)$ The tests also showed that the rapid periodic structures only enhanced the inverted interface and placing the structures on the normal interface did not improve the PL efficiency of the quantum well.

Also during this period, I encountered Gottfried Döhler who was visiting HewlettPackard from the Max-Planck-Institut in Germany. He was working on NIPI structures, which are alternating layers of $\mathrm{n}$ and $\mathrm{p}$ type materials seperated by insulating material in GaAs. These materials are similar to single heterojuction wells in that the confinement is electrostatic rather than from the crystal potential. These structures have an advantage of tunability of the quantum well energies by application of a voltage across the interface or by excition with a light source. We attempted to find an envelope state transition in these structures (NIPIEST?). No transition could be found, probably because of lack of sufficient population in the lower quantum well states.

The design of the next epitaxial growth was to be decided. Further thought on the doping mechanism lead to the realization that the ionized donors have a 
significant effect on the quantum well states. The potential terms discussed in chapter IV., section E., were assumed to be one-dimensional. But the spacing between donor ions for our doping density of $4 \times 10^{17} \mathrm{~cm}^{-3}$ becomes, on a'verage; about $135 \AA$. This is larger than the thickness of the barrier region, and so the one-dimensional assumption is not quite valid. The electrostatic potential of a charge at a distance of $r$ in GaAs is given by $\phi=e^{2} / 4 \pi \in$, which for a dielectric constant, $\epsilon$, equal to $11 \epsilon_{0}$ and a distance $r=100 \AA$ is equal to $13 \mathrm{meV}$. This potential term is random and can possibly broaden the quantum well energies. The QWEST has a much smaller broadening since both lower and upper quantum well states shift by a similar amount. But the potential could possibly also perturb the envelope wavefunction in the transverse dimensions, thus destroying the polarization selectivity of the QWEST. Evidence for the broadening effects was found in the inelastic scattering experiments $[17]$ in which the linewidth of the scattering intensity was found to reduce from 7.8 to $3.6 \mathrm{meV}$ when a $150 \AA$ thick buffer layer of undoped $(\mathrm{AlGa}) \mathrm{As}$ was inserted between the quantum well and the donor region. It was then decided to place a $150 \AA$ buffer layer on each side of the well, as shown in Fig. V.9. In addition to the reduction in broadening, it was also felt this buffer would prevent $\mathrm{Si}$ diffusion into the well region, which could also have lowered the electron density. No evidence for this diffusion is found, however, and is not expected to be an important mechanism. The evidence discussed above found that the quantum wells, because of the inverted interface, had their best mobility, photoluminescence efficiency and exciton linewidths for substrate growth temperatures between $\mathbf{6 7 0}$ and $700^{\circ} \mathrm{C}$. But Hewlett-Packard has clearly found that their best bulk material and heterostructures occurs for substrate growth temperatures of $580^{\circ} \mathrm{C}$. Also, if these results were dependent on impurities, then the best growth parameters could easily be different from one MBE machine to another. It was decided to grow this 
next set of samples at $580^{\circ} \mathrm{C}$.

Multiple quantum well of thickness $L_{w}$

\begin{tabular}{|c|c|c|c|}
\hline Layer & Thickness & Material & Doping \\
\hline Oxidation cap & $500 \AA$ & GaAs & Undoped \\
\hline Buffer region & $0.5 \mu$ & $\mathrm{Al}_{3} \mathrm{Ga}_{7} \mathrm{As}$ & Undoped \\
\hline Barrier & $50 \AA$ & $\mathrm{Al}_{.3} \mathrm{Ga} .{ }_{7} \mathrm{As}$ & $N_{D}=4 \times 10^{17} / \mathrm{cm}^{3}$ \\
\hline Dopant shield & $150 \mathrm{~A}$ & $\mathrm{Al}_{3} \mathrm{Ga}_{7} \mathrm{As}$ & Undoped \\
\hline Well & $L_{w}$ & GaAs & Undoped \\
\hline Dopant shield & $150 \AA$ & $\mathrm{Al}_{3} \mathrm{Ga}_{2} \mathrm{As}$ & Undoped \\
\hline Barrier & $100 A$ & $\mathrm{Al}_{3}{ }^{\mathrm{Ga}} .7 \mathrm{As}$ & $N_{D}=4 \times 10^{17} / \mathrm{cm}^{3}$ \\
\hline Dopant shield & $150 \mathrm{~A}$ & $\mathrm{Al}_{.3} \mathrm{Ga}_{.7} \mathrm{As}$ & Undoped \\
\hline Well & $L_{w}$ & GaAs & Indoped \\
\hline Dopant shield & $150 \AA$ & $\mathrm{Al}_{.3} \mathrm{Ga} .{ }_{7} \mathrm{As}$ & Undoped \\
\hline Barrier & $100 \AA$ & $\mathrm{Al}_{.3}{ }^{\mathrm{Ga}} .{ }_{7} \mathrm{As}$ & $N_{D}=4 \times 10^{17} / \mathrm{cm}^{3}$ \\
\hline Dopant shield & $150 \AA$ & $\mathrm{Al}_{.3} \mathrm{Ga} .{ }_{7} \mathrm{As}$ & Undoped \\
\hline
\end{tabular}

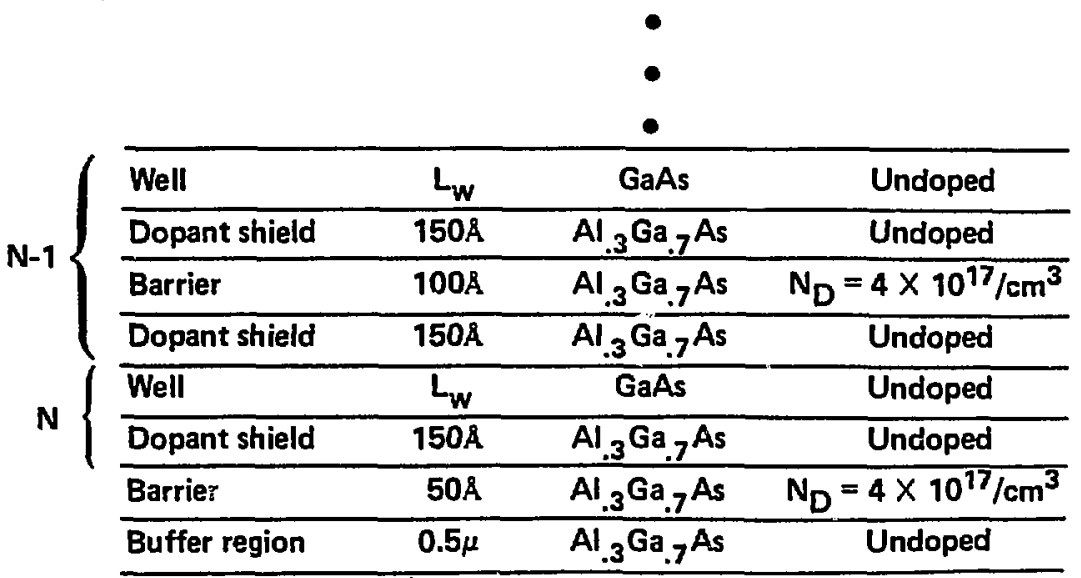

GaAs substrate undoped

Figure V.9: Layer structure of the epitaxial quantum well growth for the later samples. 
On Monday, January 23, 1984 two new samples were grown at a substrate temperature of $580^{\circ} \mathrm{C}$. The layers structure is shown in Fig. V.9, with a thickness, $L_{w}$, of 75 and $85 \AA$ for each of the 50 wells of samples B-133 and B-134, respectively. The back side of the wafer was polished on January 24, 1984 and the infrared spectrum was measured on January 25. Other than the usual absorption lines at 770 and $709 \mathrm{~cm}^{-1}$, the only other absorption seen is a narrow ( $1 \mathrm{meV}$ wide) spike with an amplitude of $6 \mathrm{mAbs}$ at a frequency of $956 \mathrm{~cm}^{-1}$ (=118 meV). This spike is on both samples and remains at full absorption strength at normal incidence of the infrared beam. The narrowness of the spike is typical of molecular absorption and the spike is attributed to a contaminant. The difference of the spectrum B-133 and $\mathrm{B}-134$ showed no structure above the $1 \mathrm{mAbs}$ level and the structure below this level had the appearance of noise. A sample of the substrate upon which these layers were grown, F620ES, was also scanned. The substrate exhibited the $770 \mathrm{~cm}^{-1}$ line, but as discussed previously the $709 \mathrm{~cm}^{-1}$ line was missing. The substrate was also missing the spike at $856 \mathrm{~cm}^{-1}$, so this contaminant must have been added later. From the photoluminescence spectra of Fig. V.8, it is noticed that the PL intesity is enhanced by about a factor of 10 by the annealing of the sample for 1 hour at $750^{\circ}$ C. This improvement suggests that some of the deep level traps thought to exist were eliminated. A similar treatment was given to samples B-133 and B-134 in the hope that the deep traps would anneal out and the electrons would then repopulate the lower quantum state. The samples were sent, along with a piece of the substrate upon which the layers were grown, to Jim Ewan at Aerotech General in Los Angeles, California. Jim Ewan first coated the samples with $\mathrm{Si}_{3} \mathrm{~N}_{4}$ to prevent the arsenic from evaporating out of the GaAs wafers and then annealed the samples at $750^{\circ}$ for one hour. The infrared spectra of these samples all had some new infrared structure, but upon subtraction of the substrate F620ES spectrum, the samples 
B-133 and B-134 exhibited no discernable signal within the $5 \mathrm{mAbs}$ level noise.

The reason for the absence of a QWEST absorption signal on these samples was unknown. The possibilities included absence of electrons from the lower quantum state, excessive broadening of the transition by poor interface structure or lack of $k$ vector conservation, or simply an error in the theory. Of these problems, an error in the theory could not be found and of the experimental problems, the lack of electrons in the lower state seemed to be the easiest to measure. However, problems had already been encountered in making contact to the wells for measurement of conductivity, and the present samples, in which buffer layers were added, were even thicker than the previous samples. A new technique was then tried. A photoresist mask was prepared with an array of Hall effect and van der Pauw geometries, as shown in Fig. V.10. The overall width of the mask as etched is about $11 \mathrm{~mm}$. The mask was oriented so that one side of each contact pad is sloped out when etched into the wafer. A Au-Ge, Ni, Au layer ohmic contact was then evaporated over each of the six ilall geometry pads and each of the four corners of the van der Pauw germetry. The metalization is seen to make contact with the edge of each of the quantum well layers in Fig. V.11. The impedance between the contacts is very high, over $10 \mathrm{Megaohm}$ in some cases. A number of anamolous effects are seen in these measurements. For instance, a 100 Volt potential across the outer two pads of the Hall effect pattern will induce a current of 1 microamp. But when the sample is placed into a 1.3 kilogauss field, the current will drop to about 20 nanoamps, when the current should not have dropped at all. If these problems are ignored, a electron density per well layer for sample B-133 is found to have values of $4 \times 10^{4}$, $4 \times 10^{7}$, and $3 \times 10^{6} \mathrm{~cm}^{-2}$ with mobilities of $2,400,3,000$, and $95,000 \mathrm{~cm}^{2} / \mathrm{V}-\mathrm{s}$, for a van der Pauw and two Hall measurements, respectively. The mobilities are within a factor of 10 of the expected mobilities. But we expected surface densities 
of $4 \times 10^{11} \mathrm{~cm}^{-2}$ for each of the layers. The measured values are too low by 4 to 7 orders of magnitude. The numerous ambiguities of these measurements did not make a convincing argument for the lack of electrons, howe-er. A better contact to the quantum wells is clearly needed. Fortunately, about this time Steve had mentioned our problems to Dan Tsui of Princeton. Dan Tsui had a microwave probe which could be used to measure the electron concentration by the magnitude of the electron cyclotron absorption in a magnetic field. Thus a contact free method could be used to measure the electron density. We sent Dan one wafer each of samples B-133 and B-134. Dan responded on May 26, 1984, with the stalement "We did cyclotron resonance measurements on your samples B-133 and B-134. Our conclus: $n$ is that there are no free carriers at all at $4.2 \mathrm{~K}$."

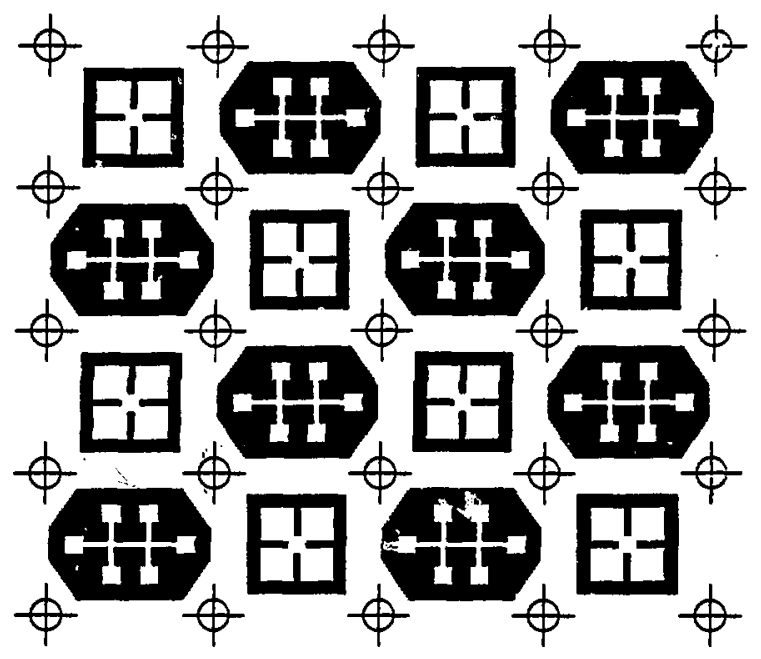

Figure V.10: Photoresist mask of Van der Pauw and Hall probe measurements to determine the electron density in the quantum well samples. The overall width of the entire pattern as etihed was about $11 \mathrm{~mm}$. Thus each probe pattern wus slightly over $1 \mathrm{~mm}$. 


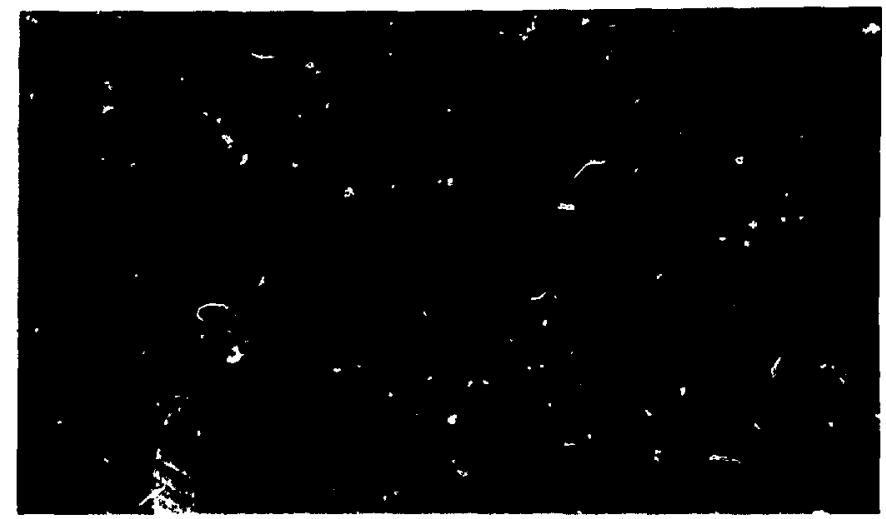

Figure V.11: An SEM photograph of the metalization overiay of a pad of a Hall probe etch in sample B-133. Note that the 7 micron deep etch clearly exposed the quantum well layers, seen as a darker coating about 3 microns deep, at an angle so as to provide some area of contact to the metalization for each of the 50 weils independently. Also notc that some of the metalization over the pas atended down the edge of the etched region from improper liftoff of this metal. A larger view shows the etcled regions shows that the metal extension cuts off completely at several spots. Also, since the upper surface is insulating, the only effect of this extra metal would be to short contact pad to one another. This was not observed.

With the above evidence, it was becoming increasingly clear that the problem with the lack of observation of the QWEST was the lack o: electrons in the lower quantum well state. It was also becoming clear that the reason for the disappearance of the electrons was because of a poor quality interface at the inverted, $\mathrm{GaAs}$ on (GaAl)As, heterojuncti n. What is not slear is the reason for this poor interface, be it dislocations or impurities. It is also not known what densities of deep level traps are generated or if their location is on the surface or in the bulk. But the evidence did indicate many improvements occured at this interface when the substrate growth temperature is increased from 580 to over $650^{\circ} \mathrm{C}$. Steve Eglash then decided to grow a number of samples with only a few wells and without the undoped (AJGa)As 
spacer layer between the well and doped region and a thin overcladding so as to facilitate measurement of the electron density by use of In diffusion into the thin layers. Hewlett-Packard also had recently installed a Reflection High Energy Electron Diffraction (RHEED) probe onto their MBE machine which could be used during the growth process. Steve then increased the growth temperature of the substrate as high as possible with the RHEED pattern continuing to indicate a good crystal structure. This temperature was about $660^{\circ} \mathrm{C}$. All the test samples were grown with $85 \AA$ quantum wells. The samples were then doped to various levels as predicted by experience with bulk materials. Sample B-271 was grown with 10 wells and Si donor density of $2.8 \times 10^{18} \mathrm{~cm}^{-3}$ in \& $100 \AA$ thick $\mathrm{Al}_{3} \mathrm{Ga}_{7}$ As barrier region around each well. The electron density was measured to be $3.5 \times 10^{13} \mathrm{~cm}^{-2}$, or $25 \%$ above the desired amount, and remained the same at both 300 and $77 \mathrm{~K}$. A second sample B-272, was grows: with pure AlAs barriers, each doped with $9.2 \times 10^{18}$ $\mathrm{cm}^{-3}$ and a total of 10 wells. The electron density was found to be independent of temperature with a value of $5.5 \times 10^{13} \mathrm{~cm}^{-2}$, or $62 \%$ of that predicted. Next, sample B-285 was grown with only 5 welis and a Si donor concentration of $5.6 \times 10^{17}$ $\mathrm{cm}^{-3}$ in a $100 \AA$ thick $\mathrm{Al}_{3.3} \mathrm{Ga}_{.7} \mathrm{As}$ barrier region. This sample had the same density at 300 and $77 \mathrm{~K}$ of $3.3 \times 10^{12} \mathrm{~cm}^{-2}$ or $18 \%$ above the predicted density. The last sample, B-286, is identical to sample B-285 except that the wells $n \in w$ have $150 \AA$ of undoped $\mathrm{Al}_{3} \mathrm{Ga}_{7} \mathrm{As}$ spacer between the well and the doped region. The measured density remained the same, at $3.3 \times 10^{12} \mathrm{~cm}^{-2}$, as the previous sample. However, the $300 \mathrm{~K}$ mobility went from 4370 in sample B-285 to $6910 \mathrm{~cm}^{2} / \mathrm{V}-\mathrm{s}$ in sample B-286. The $77 \mathrm{~K}$ mobility increased from 9720 to $52,000 \mathrm{~cm}^{2} / \mathrm{V}$-s with the addition of the spacer layers in sample B-286. The spacer leyers separating the donor ions from the quantum well states are thus seen to be of great benefit in reducing the electron scattering times. The $18 \%$ increase in electron density over that predicted 
is of the same order as the typical overgrowth and could possibly be a result of a slighty larger doping region with the same donor concentration.

With these results demonstrating that electron population of the lower quantum well states can be performed successfully, two samples were grown as above, at a temperature of about $660^{\circ} \mathrm{C}$. The structure grown is that of Fig. V.9, but with the outer $0.5 \mu \mathrm{m}$ thick buffer regions reduced to $0.28 \mu \mathrm{m}$ and the GaAs cap reduced from 500 to $50 \AA$. The samples B-331 and B-332 are grown with 50 wells of a design thickness of 85 and $75 \AA$, respectively. After growth of these samples, it was noted that insufficient In had been applied on some regions of the back surface of the GaAs wafer. The lack of thermal contact with the temperature controlled Molybdenum block allows that region to have a temperature below the $660^{\circ} \mathrm{C}$ growth temperature. A large milky haze appeared over large parts of the sample. Furthermore, micron sized hills were seen to be covering the sample with a 2 to 10 \% areal density fraction in some regions of the 2 inch diameter wafer. Because of these defects, the samples were not expected to perform well. We carefully cleaved out two $1.5 \mathrm{~cm}$ square wafers in a section of the growth which appeared to have only a small fraction of defects.

Samples B-331 and B-332 were grown on Thursday, September 6, 1984. Prior arrangements had been made with Joe Vrhel in the Applied Physics optics shop to polish the samples on Friday, September 7, and that evening the infrared spectrometer had finally been brought back to working order after weeks of problems. The infrared spectra of these samples is illustrated in Fig. V.12. A very large anomalous peak is observed on each sample and shifts to a higher energy for sample B322 , the sample with the smaller well thickness. The height of the absorption peak is about 20 mAbs. The spectrum with the infrared beam at normal incidence is shown in Fig. V.13. This anomalous peak disappears at normal 
incidence for both samples. This is indeed the QWEST.

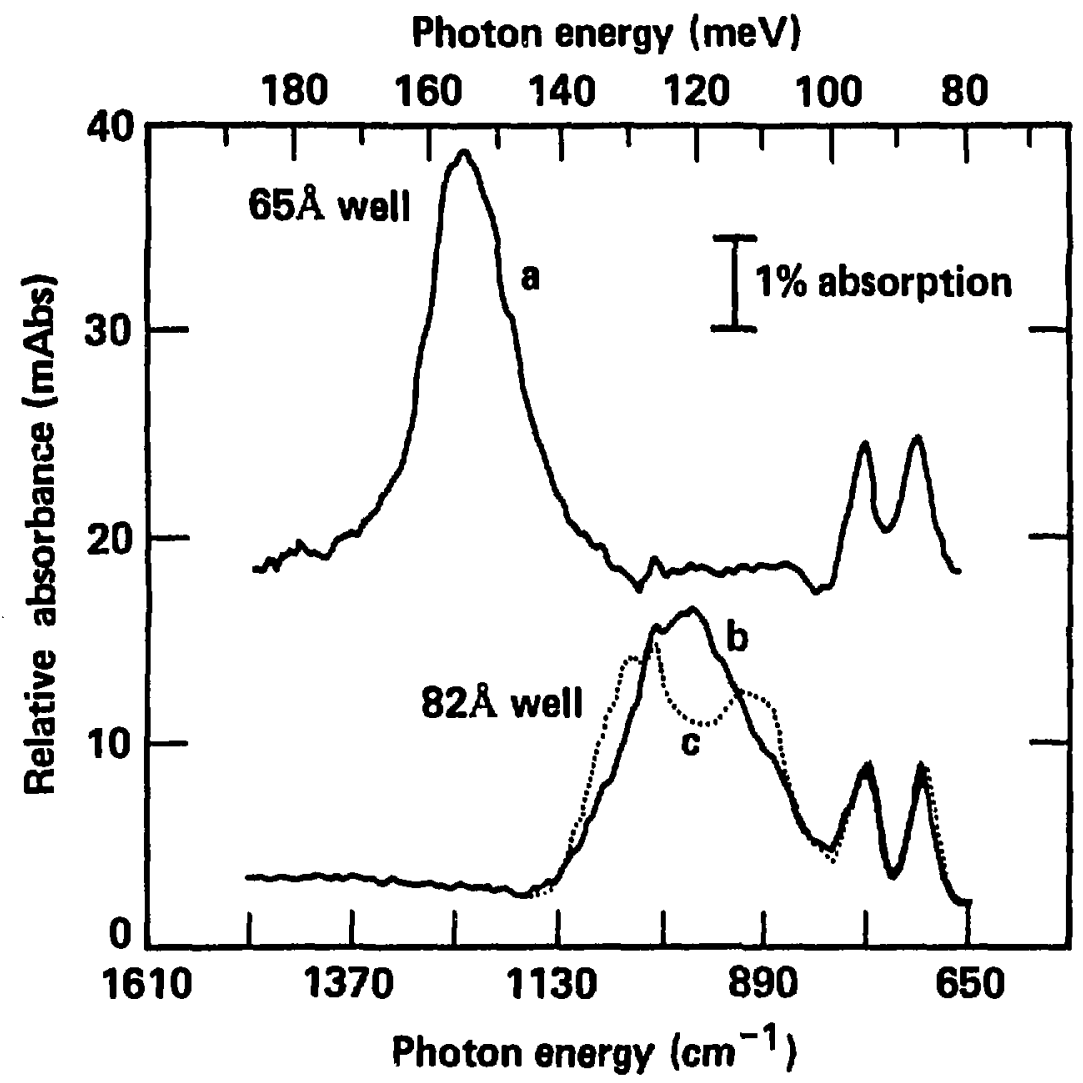

Figure V.12: Infrared absorption spectrum of sample B-331 and B-332 at $\theta=$ $73^{\circ}$ and at room temperture. mAbs $=-10^{-3} \log _{10}$ (Transmission). Spectrum (a) is of sample B-332, a $65 \AA$ quantum well, and was independent of rotation about $\phi$. Spectra (b) and (c) are both of sample B-331, a $82 \AA$ well on average, with $\phi=0^{\circ}$ (b) and $\phi=80^{\circ}$ (c), showing sample inhomogeneity with scan direction (see Fig. V.2). 


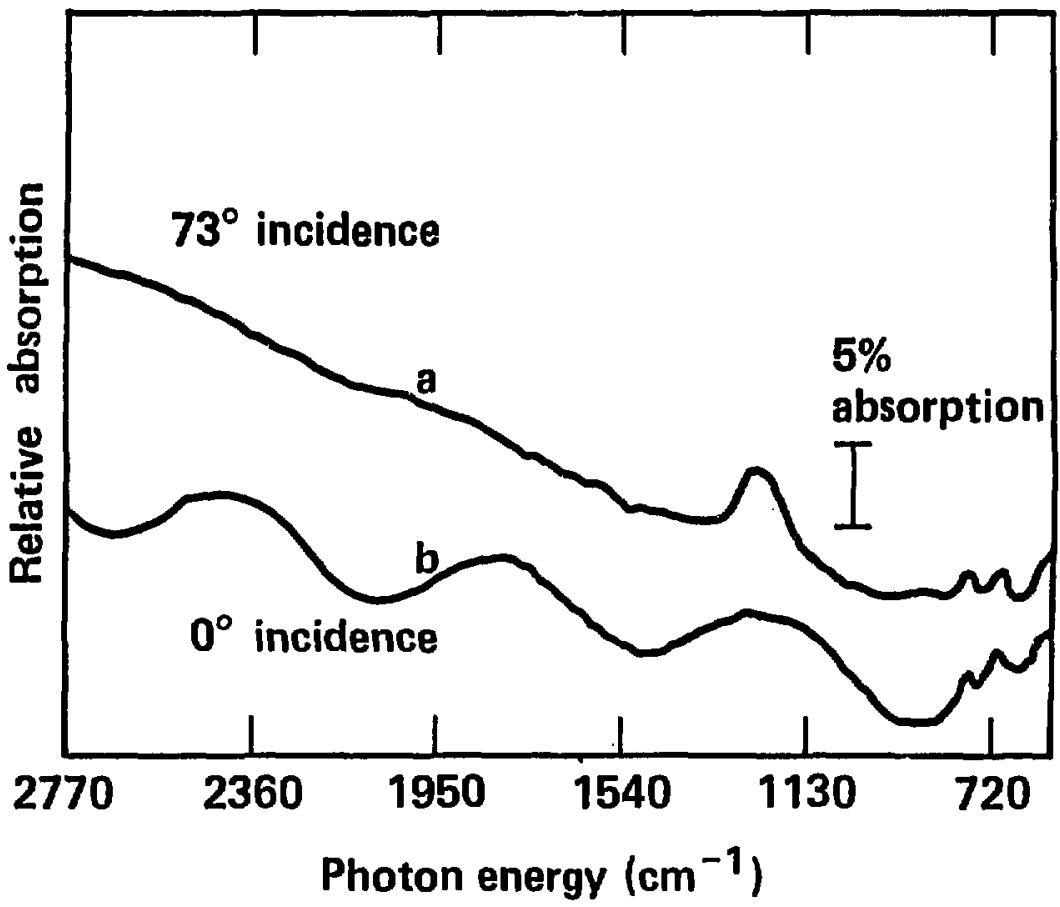

Figure V.13: Absorption spectrum of sample B-332, a $65 \AA$ quantum well, at room temperature with (a), $\theta=73^{\circ}$, and (b), $\theta=0^{\circ}$. Note the QWEST absorption peak disappears when the electric field vector of the infrared beam is rotated to lie completely in the sample plane, as expected. The shallow baseline slope is an artifact of the fourier transform process of the spectrometer in the presence of small jitter in the zero point of the interferogram, 


\section{Optical Observations of the QWEST}

The QWEST has been observed on sarnples B-331 and B-332. The anomalous peak seen on sample A-495 is now also believed to be a QWEST. The properties of this new effect are now to be carefully measured and compared with the theoretical values.

Two wafers of each sample are observed, They are labeled B-331-A, B-331-B, B-332-A, and B-332-B. The last letter refers to the specific sample of each growth. The samples are identified by either one, for specimen $A_{1}$ or two, for specimen $B$, red dots marked on the upper right corner of the sample on the side with infrared light incident with $\phi$ (see Fig. V.2) equal to zero.

The well thickness of samples B-332-A is now calibrated using the infrared epitaxial cavity technique developed in the last chapter. The lowest frequency Fabry-Perot maximum occurs at $1203 \mathrm{~cm}^{-1}$. This corresponds to a resonance with two waves in the epitaxial cavity. The thickness is found from this resonance and an index of refraction of 3.16 to be $2.63 \mu \mathrm{m}$. The sum of the specified layers should be $2.87 \mu \mathrm{m}$. The ratio of these two is 0.886 and the specified well thickness of $75 \AA$ ratios to an actual thickness of $66 \AA$. The resonance has a peak at $1231 \mathrm{~cm}^{-1}$ ( or $152.6 \mathrm{meV}$ ) with a peak height of $23 \mathrm{mAbs}$. The FWHM of the resonance is 13.4 $\mathrm{meV}$ at $\phi=0^{\circ}$ and $14.1 \mathrm{meV}$ ai $\phi=00^{\circ}$.

The sample B-332-B is taken from the edge of the 2 inch wafer and one side has a rounded smooth edge which is clearly the edge of the original wafer. The MBE machine is known to have a several percent variation in epilaxial growth rate across the wafer. This variation occurs because the molecular sources are not located on the axis of the sample and have flux variations across the surface from simple geometry considerations. In addition the edge of the crystal may have a different temperature during growth and other environmental differences which lead to a difference at the 
edge. Some of the spatial inhomogenieties can be eliminated by rotation of the sample during growth. Hewlett-Packard had just added this capability during the 9 months in which the MBE machine was down for improvements in 1983 . The rotation rate of the sample holder was limited by the controlling electronics during its initial period of insertion into the chamber to a rate less than the speed of growth of a single quantum well. (A single well typically takes about 20 to 30 seconds to grow.) Because of the inadequate spin rate of the holder, the motor was turned off for the measurement. Because of these effects the samples were expected to have a gradient in thickness. This effect was indeed observed in the edge piece. The well thickness at the center of the sample is calculated from the epitaxial cavity resonances to be $65.5 \AA$. The thickness is found to vary $7.6 \pm 0.8 \%$ across a 10 $\mathrm{mm}$ distance perpendicular to the rounded edge, with the smallest growth occuring closest to the rounded crystal edge. A $7 \%$ variation in thickness of a $65 \AA$ thick well corresponds to a variation in thickness of the well of $5 \AA$. From Fig. IV.2 this variation can be seen to correspond to a $5 \mathrm{meV}$ increase in the linewidth when the infrared beam is oriented to have the major axis of the 3 by $11 \mathrm{~mm}$ beam pattern (see Fig. V.2) on the surface oriented in this direction (which for my label happens to occur for $\phi=-90^{\circ}$ ). This increase is indeed seen to occur. At $\phi=0^{\circ}$, which corresponds to a scan parallel with the rounded edge of the wafer, the resonance occurs at an energy of $164 \mathrm{meV}$ with a FWHM of $11.8 \mathrm{meV}$. The scan with $\phi=$ $-90^{\circ}$ has a central resonance energy of $161 \mathrm{meV}$ with a bandwidth of $18.7 \mathrm{meV}$. The bandwidth in this high gradient direction is thus seen to be $6.8 \mathrm{meV}$ higher than the low gradient direction, in agreement with expectation based on energy change with well thickness. 
Spectra as a function of rotation angle

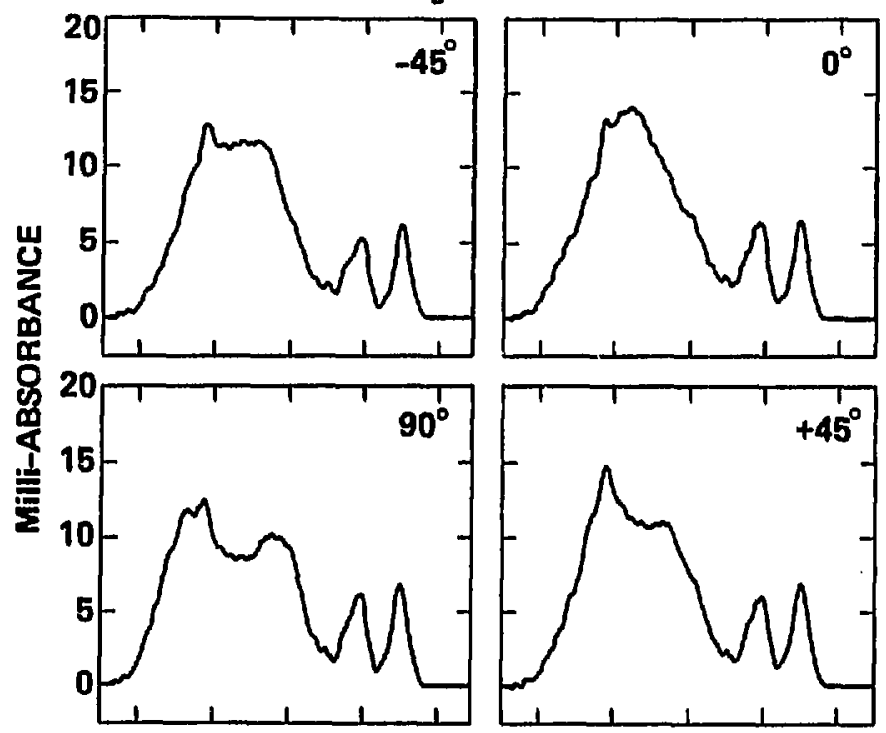

Figure V.14: Infrared absorption spectra of B-331-B at room temperature and $\theta=73^{\circ}$ as function of rotation angle, $\phi$, with $\phi$ indicated for each of the spectra.

Samples B-331-A and B-331-B have very broad energy bands. The samples appear to have similar behavior so we only discuss sample B-331-B. A maximum in the epitaxial cavity resonance occurs at an frequency of $1088 \mathrm{~cm}^{-1}$. The epitaxial thickness then becomes $2.81 \mu \mathrm{m}$. The design layers add to $3.02 \mu \mathrm{m}$. If we scale the design $85 \AA$ well thickness by the same proportion, the actual well thickness becomes $82 \AA$. The spectra of this sample has very broad linewidths, as shown in Fig. V.12. Furthermore, as shown in Figs. V.12 and V.14, the spectra are highly sensitive to the scan angle, $\phi$. A hypothesis that this variation might be due to irregularities on the surface was investigated by scanning the thickness of the epitaxial layers over the surface. Suprisingly, the surface was found to be extremely flat with only a $2 \%$ variation in thickness found in one direction $\phi=0^{\circ}$ over a distance of $10 \mathrm{~mm}$ and 
no gradient at all found in the orthogonal direction $\phi=90^{\circ}$. The spectra all show a central peak at $121 \mathrm{meV}$ witì a FWHM of 21 to $30 \mathrm{meV}$, depending on the rotation, $\phi$, of the sample. If approximately $10 \mathrm{meV}$ of the broadening is assumer to occur even for perfectly uniform wells, then the excess broadening of these lines would be about 10 to $20 \mathrm{meV}$. From Fig. IV.2 it can be seen that a nonuniformity of up to $20 \%$ would be required to explain the excess linewidth. But the epitaxial growth was found to be an order of magnitude more uniform. In fact the uniformity of the epitaxial layers of this sample, B-331-B, is several times better than that of sample B-332-B, which has a much narrower linewidth in spite of its larger gradient. The explaination to this phenomena was found when Steve recalled that he had stopped the rotation of the sample during growth differently for samples B-331 and B-332. For sample B-331 he simply turned the motor speed to zero, wheras for sample B-332 he turned off the power to the motor. It is known that the motor speed when turned to zero does not go exactly to zero, and some rotation of an unknown amount occurs during 4 hours of the epitaxial growth. However, if only one rotation or more occured during the 4 hour growth, then each region of the wafer got a selection of all possible well widths within the growth region. The sample B-332-B above, held stationary during the growth, has a thickness gradient of $7 \%$ per centimeter. If linearly extrapolated across the $50 \mathrm{~mm}$ diameter wafer, then the wafer could have an edge to edge variation in thickness of $30 \%$. Thus a region travelling around the chamber on an inner radius could easily have a $20 \%$ variation from one well width to another. The sample would also appear to have a better than normal uniformity of the overall surface because of the averaging effects of the rotation. This inadvertant rotation thus explains the contradictions of sample B-331.

Low temperature experiments were performed in an attempt to beiter understand the broadening mechanisms of the QWEST. Because the broadening of the 
QWEST of sample B-331 was obviously determined primarily by inhomogeneities, the low temperature measurements focused on sample B-332-A. The sample B-332$A$ is found to have a sightly reduced linewidth of $10.6 \mathrm{meV}$ at room temperature when it is oriented at an angle of $\phi=45^{\circ}$, and this is the orientation used to probe the low temperature properties of this sample. The room temperature QWEST has a resonant energy of $152.3 \mathrm{meV}$ and peak heigb' of $30 \mathrm{mAbs}$ with a linewidth of 10.6 meV. As the temperature is reduced to $34.5 \mathrm{~K}$, the resonance energy of the QWEST increases to $156.2 \mathrm{meV}$, the peak increases to $43 \mathrm{mAbs}$, and the linewidth decreases to $7.2 \mathrm{meV}$. The small variation in these quantities as a function of temperature is linear to within the accuracy of the measurement. Note the product of peak height and linewidth stay constant within $3 \%$ throughout the temperature region, indicating the electron density and oscillator strength remain constant with temperature. The spectra of the QWEST at several temperatures are shown in Fig. V.15. The $224 \mathrm{~K}$ QWEST resonance is compared to the $34.5 \mathrm{~K}$ spectra in an expanded plot in Fig. V.16. The full absorption spectra, from $4000 \mathrm{~cm}^{-1}$ (or $2.5 \mu \mathrm{m}$ in(rared wavelengths) to $400 \mathrm{~cm}^{-1}(25 \mu \mathrm{m}$ in(rared wavelengths), is shown in Fig. IV.17 for two temperatures for sample B-332-A. The two traces are untouched for the purpose of showing actual noise of the full spectrum. The high frequency fuzz at the higher wavelengths is unfiltered cavity resonances of the full GaAs wafer. This spectra can be compared to that of sample A-516 in Fig. V.3. 


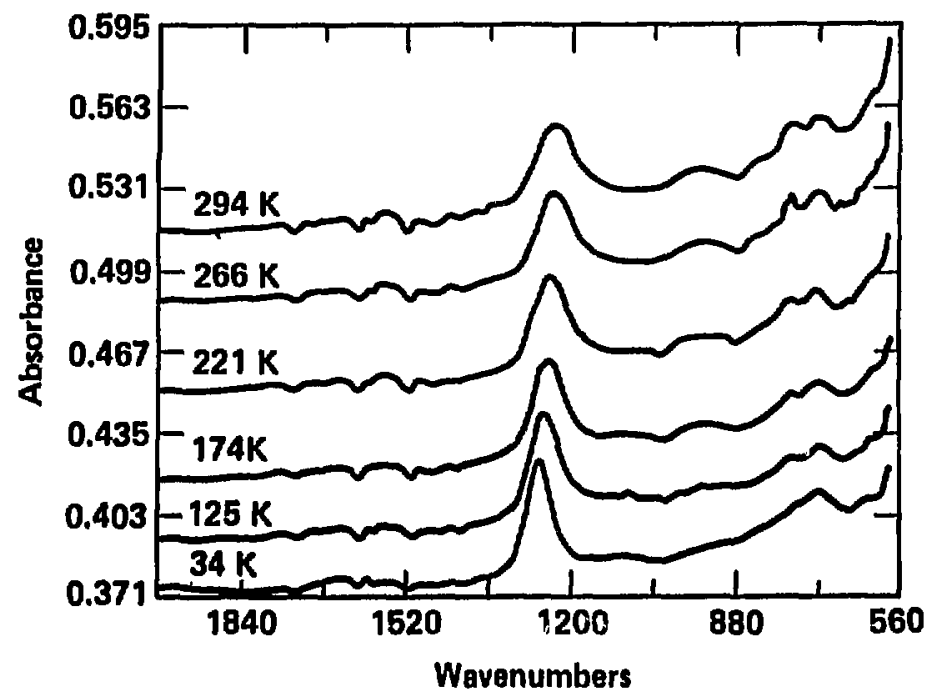

Figure V.15: Infrared absorption spectra of sample B-332-A at $\theta=73^{\circ}$ and several temperatures.

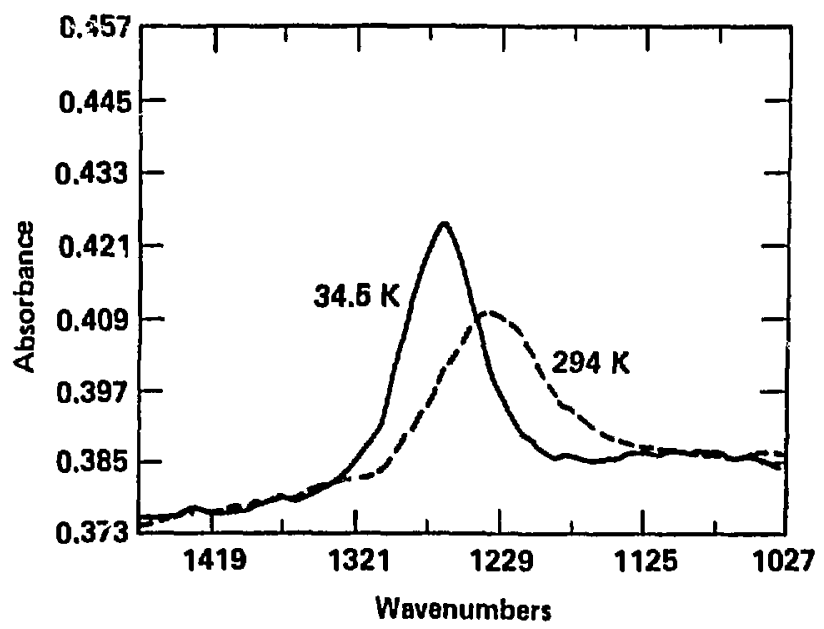

Figure V.16: Infrared absorption spectra of B-332-A at $\theta=73^{\circ}$ for 34 and 294 K. 


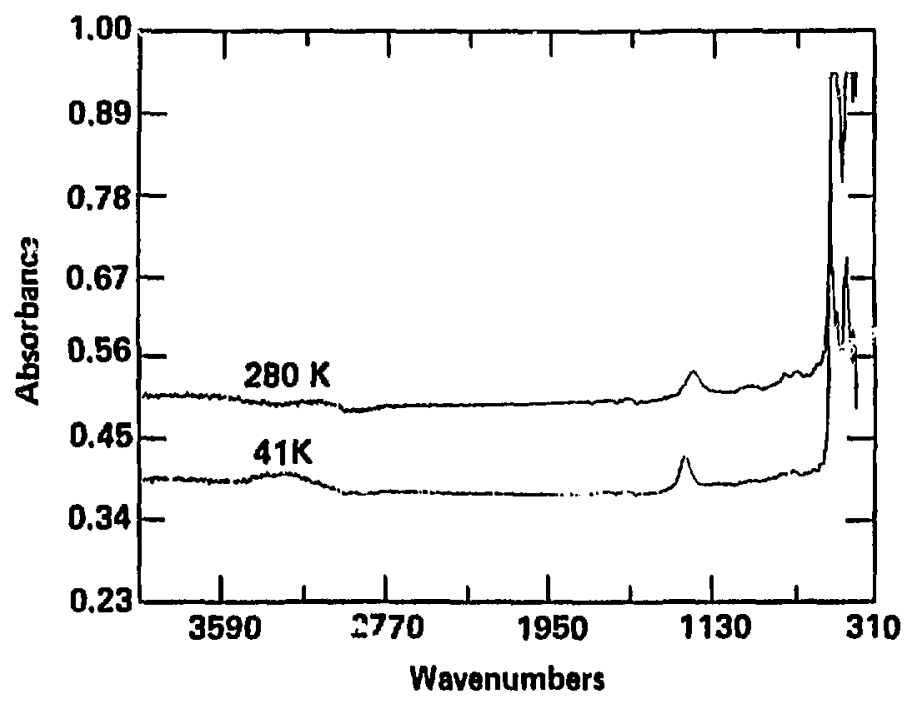

Figure V.17: Infrared absorption spectra of B-332-A at $\theta=73^{\circ}$ for 280 asd $41 \mathrm{~K}$. This spectra is not redrawn so as to retain the original noise of the GaAs substrate and epitaxial layers. Note that excep" for the QWEST, the spectrum is devoid of absorption peaks at this level. The noise present is from two sources. The first is residual $\mathrm{CO}_{2}$ in the spectrometer, which is the source of the broad bumps at $3000 \mathrm{~cm}^{-1}$ for the $41 \mathrm{~K}$ plot and the numerous peaks around $1600 \mathrm{~cm}-1$. The $\mathrm{CO}_{2}$ spectrum actually consists of numerous $1 \mathrm{~cm}^{-1}$ wide peaks, but the spectra here are taken with too low a resolution to see the individual peaks. In addition, high frequency oscillations, from the cavity resonance formed by reflections of the outer wafer surfaces which are not ruite removed at Brewster's angle, are seen at the higher wavenumbers.

The oscillator strength can be found from equation (V.11), which gave the oscillator strength from the integral of the line over optical frequency. It was found 
that $I A F=0.218 \mathrm{f} \mathrm{Abs-cm}^{-1}$. A computer integral of the QWEST of sample B332-A at 0 and 90 degrees rotation $\left(\phi=0,90^{\circ}\right)$ found integrals of 2.754 and 2.715 Abs-cm ${ }^{-1}$, respectively. These integrals give an oscillator strength of 12.6 and 12.5 for the two rotations. For sample B-332-B, the integral of the line shape is found to be 2.277 and $1.683 \mathrm{Abs}-\mathrm{cm}^{-1}$ for rotation angles of 90 and 0 degrees. These integrals result in an oscillator strength of 10.4 and 7.7 for the two angles. It should be noted that the measurements assume the electron density per quantum well is $4 \times 10^{11} \mathrm{~cm}^{-2}$. However we saw that for sample B-286 the electron density had $18 \%$ extra electrons, and our samples could have similar inaccuracies. If the electron density is assumed to scale by the size of the doping region, the above numbers for the oscillator strength increase by $15 \%$ to as high as 14.5 . The abnormally low oscillator strength of the second sample indicate some of the electrons are missing.

The integral of the lineshape of sample B-331-B, as probed on October 31, 1884, at rotational angles oi $\mathbf{- 4 5}, \mathbf{4 5}, 0$, and 80 degrees found oscillator strengths of 10.0 , $11.5,11.6$, and 11.4 respectively. It should be noted that three weeks earlier, on October 11, the same spectra was measured with a weaker lineshape. See Fig. V.18. On Oct. 11, the oscillator strength at rotation angles of 0 and 80 degrees was found to 7.16 and 8.81 . The increase in the oscillator strength is probably because of an increase in the number of electrons in the lower quantum well state that were previously trapped elsewhere. Thus the larger numbers of Oct. 31 are probably the more accurate numbers, for the oscillator strength. The larger linestrengti of Oct. 31 also appears more consistent as a function of rotation angle. 


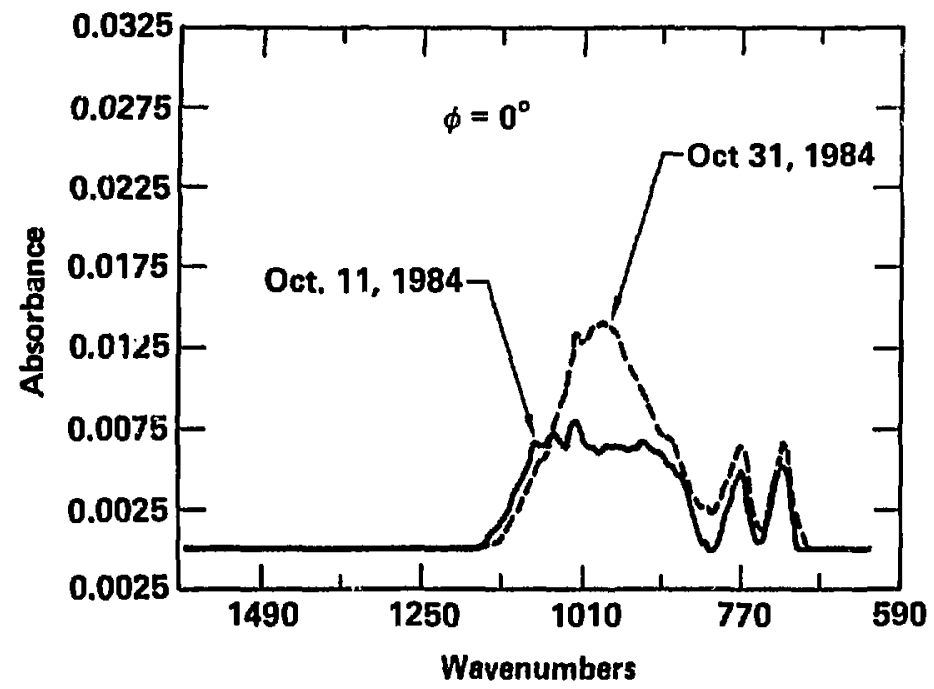

Figure V.18: The infrared absorption spectrum of sample B-331-B at $\theta=73^{\circ}$ and $\phi=0^{\circ}$ on two different dates.

Other changes in lineshape are noticed in several spectra. In Fig. V.10, the spectrum of sample B-332-A on two different dates is noticed to have shifted $6 \mathrm{meV}$ from $152 \mathrm{meV}$ to $146 \mathrm{meV}$. At a later date, on Feb. 28, 1885, this resonance shifted back to the original position at $152.4 \mathrm{meV}$, but the oscillator strength changed from 12.6 on Oct. 11, 1983 to 11.2 on Nov. 3, 1983 to 11.3 on Feb. 28, 1985. If we use the measurement of oscillator strength as an indication of electron density, undei the assumption that the true oscillator strength remains constant, we find that the energy shift is unrelated to the changes in electron density. 


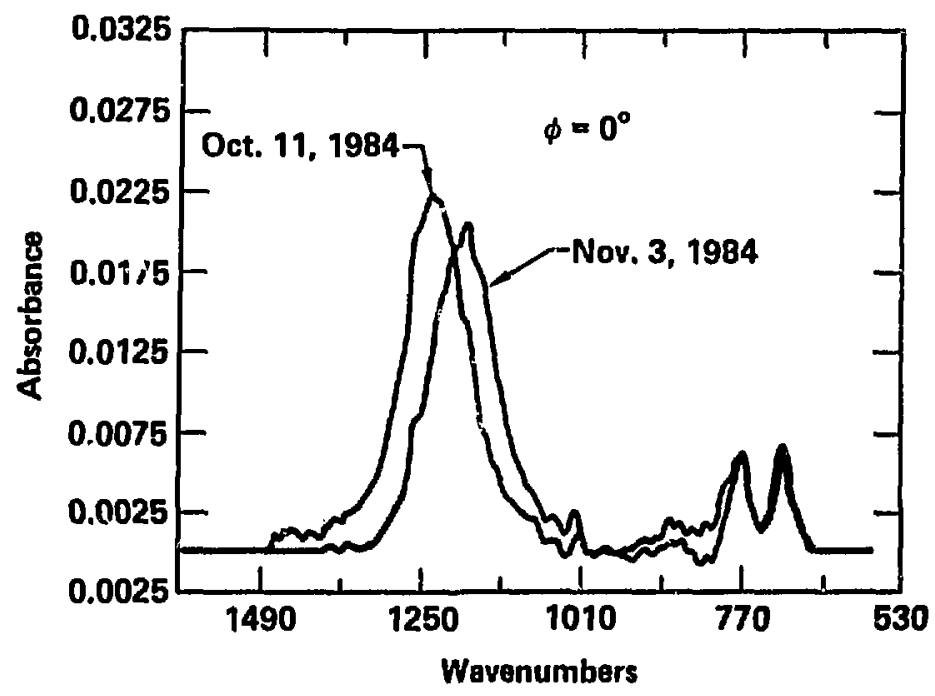

Figure V.19: The infrared absorption spectrum of sample B-332-A at $\theta=73^{\circ}$ and $\phi=0^{\circ}$ on two different dates. 


\section{Analysis of Observations}

The infrared measurements of the QWEST yield three parameters for analysis. These are she QWEST energy, oscillator strength, and bandwidth. These parameters are measured both as functions of well width and sample temperature. One observation is also seen with different quantum well crystal structure. These observations are compared with the theoretical predictions of the previous chapter and used for further analysis of the quantum well states.

The first parameter measured is the QWEST energy. This energy was seen in chapter IV to be very nearly equal to the difference in energy of the two lowest energy conduction band quantum well states. Unlike exciton measurements, these energy measurements are independent of band gap and valance band quantum well energies. The predicted QWEST energies, from Tabje IV.3, are compared with the observed energies, given in the previous two sections, in Table V.1.

\section{Table V.1}

Comparison of observed QWEST energ. s with theoretical expectation. Energie . are measured in meV.

Sample Well Thickness Predicted Energy Observed Energy

$\begin{array}{cccc}\text { B-332-A } & 65 \AA & 142.4 & 152 \\ \text { B-331-A } & 82 \AA & 117.8 & 124 \\ \text { A-495 } & 92 \AA & 105.0 & 110\end{array}$

The observed QWEST energy peak wandered over a range of about $6 \mathrm{meV}$ within a several week time frame. This wandering of energy was associated with a change of absorption strength, but interestingly, the bandwidth of the QWEST did not change. This behavior of the QWEST currently has no explanation. Furthermore, 
a shift in quantum well energies has never been reported by any of the many other types of probes as mentioned in chapter II. However, those interband measurements include a large energy from the bandgap which changes rapidly with tenperature ${ }^{[45]}\left(0.5 \mathrm{meV} /{ }^{\circ} \mathrm{C}\right)$. Thus it cannot be certain if the quantum well energies change over time and merely have not been noticed or else this energy wander is unique to the QWEST. The observed energies listed above are either the central energy or the most stable energy of the QWEST during the wander. The detailed behavior of this wander was presented in the previous two sections.

The predicted energies in Table V.1 are seen to be consistently about 5 to 10 meV below the observed energies. The predicted energies in Table V.1 were based on continuity of the derivative of the envelope wavefunction across the well boundary. If wa use the alternative boundary condition, with continuity of this derivitive multiplied by the inverse effective mass assumed, then the predicted energies are yet lower still. But then the change of QWEST energy with well thickness is in better agreement. The predicted energies under the two assumptions are both plotted in Fig. IV.2, along with the experimental points. The approximately 5 $\%$ error between the predicted and observed energies could have several causes. The largest theoretical problem is the treatement of envelope wavefunction at the boundary between the GaAs well and the (AlGa)As barrier. This boundary condition determines entirely how the quantum well states are quantized, and the QWEST energy depends highly on this treatement. Fortunately, the well and barrier materials are sufficiently similar that simple continuity conditions on the envelope and its derivitive have been found to give fair agreement with observations. [3] Furthermore, the discrepencies between observed and predicted QWEST energies can be entirely explained by uncertainties in the input quantum well parameters. For instance, the beight of a $\mathrm{Al}_{.3} \mathrm{Ga}_{.7} \mathrm{As}$ barrier to the $\mathrm{GaAs}$ well was only recently 
discovered [23] to be near $240 \mathrm{meV}$ instead of $320 \mathrm{mev}$, as had been thought for many years. ${ }^{[3]}$ This difference in barrier height can change the QWEST energy by about $20 \mathrm{meV}$, and the true height is still being refined. Also, the calculation of the band energy of a conduction band electron using $k \cdot p$ theory neglected the higher order effects from other bands. Although the band energy could be renormalized to first order by use of the empirical effective mass of GaAs, the deviations of true bands from the effective mass approximation lowers the QWEST energy by about $10 \mathrm{meV}$. The error in this deviation is estimated to be about $30 \%$, so this uncertainty could be the source of another $3 \mathrm{meV}$ error. Finally, an unknown effect is causing the QWEST resonance to wander over a $6 \mathrm{meV}$ range. Until this effect is understood, it is impossible to make a prediction with any greater accuracy than the magnitude of wander. Thus the theoretical treatment of the quantum wells used here provides values of the energy, oscillator strength, and bandwidth as accurate as possible with the present knowledge of the well structure.

The QWEST energy was measured as a function of temperature for the sample B-332-A. The QWEST energy was found to increase from 152.3 to $156.2 \mathrm{meV}$ as the temperature was reduced from $300 \mathrm{~K}$ to $35 \mathrm{~K}$. Since the thermal expansion coefficient of GaAs is about $6.7 \times 10^{-6} /{ }^{\circ} \mathrm{C}$, the well size only increases by about $0.2 \%$ or $0.1 \AA$ for a $270 \mathrm{~K}$ temperature rise. This small thickness change has a negligible effect on the quantum well energies. However, the inverse effective mass of $\mathrm{GaAs}$ drops from 15.9 to 14.9 (in units of $1 / m_{e}$ ) as the temperature drops from $300 \mathrm{~K}$ to $35 \mathrm{~K} .{ }^{[22]}$ If the barrier height remains constant, this mass change predicts a $4 \mathrm{meV}$ drop in the QWEST energy of sample B-332-A as the temperature is reduced from $300 \mathrm{~K}$ to $35 \mathrm{~K}$. The barrier height does not remain constant, however. The band gap of $\mathrm{Al}_{3} \mathrm{Ga}_{7} \mathrm{As}$ is found to increase at a greater rate than the band gap of $\mathrm{GaAs}$ as the temperature is reduced.(10) The increase of the barrier bandgap 
over that of the well bandgap as the temperature is reduced from $300 \mathrm{~K}$ to $35 \mathrm{~K}$ is ${ }^{40]}$ about $20 \mathrm{meV}$. If the fraction of the band gap discontinuity which becomes a barrier to a conduction band electron remains constant at $65 \%$, as reported in some observations, [23] then the increase in barrier beight is only $13 \mathrm{meV}$. This small an increase in barrier height only increases the QWEST energy by about $2 \mathrm{meV}$. If the effective mass change is included, the predicted net change is a QWEST energy reduction of $2 \mathrm{meV}$, in contrast with the observation of a $4 \mathrm{meV}$ increase, as the temperature is lowered from 300 to $35 \mathrm{~K}$. This QWEST energy temperature dependence can be explained by a $40 \mathrm{meV}$ increase in the barrier height. However, this $40 \mathrm{meV}$ increase in barrier height would require the fraction of the bandgap) difference which is a barrier to the conduction band electron increase from $65 \%$ to $70 \%$ as the temperature is reduced from $300 \mathrm{~K}$ to $35 \mathrm{~K}$. Since this fraction is not known at any temperature to this $5 \%$ accuracy, a temperature dependence of this size cannot be deduced from present observations. [23]

The second parameter of interest is the QWEST bandwidth. The suspected effects of various broadening mechanisms are tabulated in Tables IV.1 and IV.2 for a $65 \AA$ thick well at temperatures of 300 and $35 \mathrm{~K}$ respectively. The bandwidth was predicted to be about $8.7 \pm 3.3 \mathrm{meV}$ at room temperature and reduce substantially to $4.9 \pm 3.0 \mathrm{meV}$ at $35 \mathrm{~K}$. The sample $\mathrm{B}-332-A$, a $65 \AA$ quantum well, was observed to have a bandwidth of 10.6 and $7.2 \mathrm{meV}$ at temperatures of 300 and $35 \mathrm{~K}$, respectively. The behavior of these broadening mechanisms as a function of temperature, as shown in Tables $\mathrm{N} .1$ and $\mathrm{N} .2$, shows discrepencies between prediction and observation. In particular, a net broadening is predicted which is too smail by about $2 \mathrm{meV}$. If we look at the low temperature linewidth, the density of states of the QWEST, which is dominant at higher temperatures, becomes fairly small (2.6 meV) and more accurate. The other two broadening effects were assumed to be 
independent of temperature. The inhomogeneous broadening is mostly from transverse variation of the well width with distance, which was measured, as described in the previous section, to be in agreement to the assumptions of Tables IV.1 and IV.2. Thus this term is thought to be accurate. The last term, from lifetime broadening, has large theoretical uncertainties. Furtbermore, the other terms are iairly accurate and are too small to come close to explaining the low temperature linewidth. The low temperature linewidth is therefore suspected to reflect the lifetime broadening, and results in a lifetime broadening of about $6 \mathrm{meV}$, near the maximum expected. This bandwidth corresponds to a lifetime of 0.2 picoseconds. If Tables IV.1 and N.2 are changed to reflect this interpretation, then they become as given in Tables V.2 and V.3.

\section{Table V.2}

A possible interpretation of the broadening effects for a $65 \AA$ quantum well at $300 \mathrm{~K}$,

\begin{tabular}{ll} 
Density of QWEST states & $7.5 \pm 2.0 \mathrm{meV}$ \\
Lifetime broadening & $6.0 \pm 0.0 \mathrm{meV}$ \\
Inhomogeneous well width & $3.0 \pm 1.5 \mathrm{meV}$ \\
\hline & $10.1 \pm 2.0 \mathrm{meV}$
\end{tabular}




\section{Table V.3}

A possible interpretation of the broadening effects for a $65 \AA$ quanturi well at $35 \mathrm{~K}$.

$$
\begin{array}{ll}
\text { Density of QWEST states } & 2.4 \pm 0.6 \mathrm{meV} \\
\text { Lifetime broadening } & 6.0 \pm 0.0 \mathrm{meV} \\
\text { Inhomogeneous well width } & 3.0 \pm 1.5 \mathrm{meV} \\
\hline \text { Total } & 7.1 \pm 0.5 \mathrm{meV}
\end{array}
$$

This explanation, which was found neccesary to properly describe the low temperature data, is also seen to correctly describe the $300 \mathrm{~K}$ bandwidth.

The bandwidth of sample B-331, with approximately $82 \AA$ thick wells, is not discussed since its bandwidth is clearly broadened by an inadvertant variation in well thickness from well to well during the growth of the 50 wells. This gave a bandwidth for this sample of over $20 \mathrm{meV}$ and nearly $30 \mathrm{meV}$ for some orientations of the wafer.

The above bandwidth discussion assumed the ionized donors in the barrier region were sufficiently far away from the quantum well so as not to affect the envelope wavefunction and energy states. The growth of the above two samples, B-331 and B-332, had an undoped barrier region $150 \AA$ thick separating the ionized donors from the well region for this purpose. The effect of these ionized donors is found by examination of sample A-495. This sample bad no undoped region in the barrier separating the well from the ionized Si donors. As discussed in the previous section, the fluctuation of this potential is of order $10 \mathrm{meV}$. However, the effect of this fluctuation on the QWEST bandwidth is anticipated to be much smaller for two reasons. First, the potential affects both lower and upper quantum well energies by 
a similar amount. Second, the electrons will tend to concentrate in the lower energy regions, so regions observing the full fluctuation of the potential will not be equally populated. The latter point has another important consequence. If the electrons tend to concentrate in the lower energy regions of the two-dimensional surface, and the average distance between donors is $140 \AA$, then the envelope wavefunction will have some structure in this transverse dimension with a similar size variation. Since this $140 \AA$ variation in envelope wavefunction is much smaller than the infrared wavelengths, this perturbation could easily lead to a small dipole in the transverse dimension. The QWEST would then have a nonzero absorption strength in the other two dimensions.

The observations of sample A-495 saw a QWEST absorption with a $10.5 \mathrm{meV}$ linewidth, similar to the sample B-332-A. But since sample A-495 contains $82 \AA$ wells, the density of QWEST energy has a predicted FWHM of $5.6 \mathrm{meV}$, which is $2 \mathrm{meV}$ smaller than that of sample B-332, seen to have a $7.5 \mathrm{meV}$ density of states bandwidth. If the net bandwidth is taken as the sum of squares of the individual terms, and we take the other terms in Table V.2, besides the density of states, to be the same, then we find the ionized donors contribute an additional term with 6 $\mathrm{meV}$ broadening. It should be emphasized that this value is obtained by taing the difference of the squares of large terms which are themselves uncertain, and should not be considered to be very accurate. The absorption strength was also observed to drop by a factor of about 3 when the polarization of the infrared beam was rotated to lie in the well plane by changing the incident angle of the beam from Brewster's angle to normal incidence. But theoretically, the absorption strength should have dropped to identically zero, as observed in samples $\mathrm{B}-331$ and $\mathrm{B}-332$. In section $\mathrm{A}$ of this chapter, the electric field of the infrared probe was shown to lie almost entirely in the plane of the quantum well. The coupling of the field to a dipole normal to 
the well, as we expect the QWEST to be, is thus 10 times smaller than the coupling to a similar size dipole in the plane of the well. The factor of 3 reduction in tie absorption strength for this polarization actually indicates a reduction of 30 in the oscillator strength in this direction when the fields are taken into account. The effect of placing the ionized donors in the vicinity of the well is therfore suspected to add a broadening term of about $6 \mathrm{meV}$ and convert about $3 \%$ of the oscillator strength to a direction in the plane of the quantum well.

The remaining optical parameter of interest is the oscillator strength of the QWEST. This strength was difficult to measure because the electron density in the well was difficult to obtain, measure, and maintain. Thus the absorption strength would vary significantly. The samples $\mathrm{B}-331$ and $\mathrm{B}-332$ were finally developed with some stability in this electron density. The problem with the electron density in the lower state was always found to be one of disappearance rather than an excess. This behavior is consistent with lower energy bound states being formed which can trap an electron from the lower quantum state. Because of this behavior, it was felt the maximum oscillator strength found for a given sample was probably the most accurate. The maximum value obtained for sample B-331 was found to be 11.6 and that for sample B-332 was 12.6. The oscillator strength predicted for these two samples is 13.2 and 12.1 , respectively. The experimental values, which are uncertain to about $10 \%$ because of inaccuracies in doping density, are thus seen to be in excellent agreement with prediction. If the experimental values for the oscillator strength and energy are used with the aid of the definition of the oscillator strength in equation (IV.50), then the dipole matrix element is found to be $18.8 \mathrm{e}-\AA$ and 17.8 e- $\AA$ for samples $\mathrm{B}-331$ and $\mathrm{B}-332$, respectively.

The optical parameters of the QWEST are seen to match well with prediction and good understanding of thier behavior has been found. Further work and more 
samples is sure to refine this understanding. Some uncertainties still lie in the fabrication of samples without electron traps, numbers for well parameters such as barrier height, and the lifetime of the upper state, among others. These problems are also of current interest to those involved with quantum well samples and will undoubtedly be solved in the near future. 
DX. Conclusion and Future Applications

An infrared dipole transition between two envelope siates within the same band of a quantum well is predicted and observed. This transition is called a Quantum Well Envelope State Transition or QWEST.

The QWEST is now very clearly shown to be realizable in the conduction band of a (AlGa)As quantum well. The observation of a QWEST is seen on three different samples with quantum well thicknesses 65,82 , and $02 \AA$ and resonant energies of 152,121 , and $108 \mathrm{meV}$, respectively. The observed resonant energies are in good agreement with theory. The oscillator strength is found to have values of over 12 , in good agreement with prediction. The linewidths are seen as narrow as $10 \mathrm{meV}$ at room temperature and $7 \mathrm{meV}$ at low temperature, thus proving a narrow line resonance can indeed occur between transitions of free electrons. Techniques for the proper growth of these quantum well samples to enable observation of the QWEST have also been found using (AlGa)As compounds.

The QWEST has a quantum dipole as large as $20 \mathrm{e} \AA$. The transition also has a narrow bandwidth, an anomaly for transitions between free electrons. This large dipole and narrow bandwidth result in strong nonlinear optical properties. For instance, the ratio of index of refraction change to incident infrared intensity, $n_{2}$, can be as high as $10^{-5} \mathrm{~cm}^{2} /$ Watt. In addition, both the lower and upper quantum well states consist of free electrons in the same conduction band. An electron can thus relax from the upper to the lower energy state by an elastic scattering, requiring no energy transfer. This property enables the transition to have an extremely fast transition time, predicted to be subpicosecond.

The motivation for the development of the QWEST was its application to the development of a digital computer based entirely on optics, with no electronics required. Advantages for an optical system over electronic are seen in both the 
elimination of inductance-resistance-capacitance problems and in the elimination of electron transit times through small channels. A discussion of optical computing is given in Appendix B.

The importance of the QWEST to the physical operation of a logic element lies in its large dipole, which gives it a very large nonlinear coeficient. Of interest to optical logic is the $n_{2}$ coefficient, which varies as the dipole to the fourth power (see chapter IV, section F). The dipole of the QWEST is 100 times larger than the dipole of an atomic state, giving an improvement in the $n_{2}$ coefficient by a factor of $10^{8}$ over atomic systems. In addition, the currently proposed implementations of an optical logic element in the solid state are limited in speed by the relaxation rate of an electron from one band to another, with relaxation times in the 10's of nanoseconds. ${ }^{[51,52]}$ The QWEST has a subpicosecond relaxation time, much faster than any of today's nanosecond logic.

In addition to the physical properties of the QWEST, which gives it a high nonlinearity and fast response time, the QWEST also has a number of properties which are extremely important to the engineering of integrated optical logic elements into a inexpensive system. (See Appendix B.) One of the properties of the QWEST important to optical logic is its tunabilty in the infrared. Infrared wavelengths are considered essential to integrated optics for several reasons. First, the larger wavelengths allow easy fabrication of integrated optical elements and waveguides by use of standard photolithographic processes. Second, many very inexpensive and very high efficiency lasers exist in the infrared. Two examples are the $\mathrm{CO}_{2}$ and $\mathrm{CO}$ lasers, with wavelengths of 10 and 5 microns, respectively, wallplug efficiencies as high as $\mathbf{3 0 \%}$, and prices as low as $\mathbf{\$ 5 0}$ per watt of output for high power lasers. Third, the GaAs materials are very transparent in the infrared. Furthermore, Germanium, a material which grows well on GaAs and is important to 
the fabrication of integrated optical elements, only transmits light with wavelengths greater than 2 microns.

The tunabilty of the QWEST is also important to optical logic. First, the transition can be tuned to match any laser in the infrared, allowing use of the best available laser source throughout the region. Second, as an optical computer is scaled to smaller sizes to achieve higher speeds, the scaling to a smaller wavelength, necessary to make smaller structures, is allowed by the QWEST. Third, the QWEST resonance can be placed on both sides of the laser frequency. A given logic element can then use ncnlinearities of both signs simultaneously. This exira freedom is important in fabrication of logic elements which preseut zero reflection to the input signals for all levels of output, for instance.

In conclusion, the discovery of the QWEST in GaAs is a breaktilrough in the construction of an all optical digital computer. The advantages offered by a logic element based on the QWFST are similar to those of the transistor when first developed, namely, a factor of $\mathbf{1 0 0 0}$ in both speed and power simultaneously, for a million-fold reduction in the speed-time product. The break through is not only because of the physical effect and all the properties such as nonlinearity and speed associated with it, but also the engineering properties of the जaAs QWEST, which allows construction of an optical logic element and assor,ated interconnects and elements into a full optical digital computer system, all highly leveraged off of existing GaAs technology. My original goal, which lead to the discovery of the QWEST, was the development of an all optical computer. The developments are far surpassing what I thought, even with my wild iders, would be possible. However, the results presented in this thesis are only the opening through which the rapid advances towards optical logic can now proceed, which is the direction I am now taking. The QWEST has only begun. 


\section{References}

[1] L.I. Schif, "Quantum Mechanics," McGraw Hill,(1868)

[2] Gordon Baym, "Lectures on quantum mechanics," W. A. Benjamin, Inc., Advanced Book Program, Reading, Massachusetts (1960)

[3] R. Dingle, "Confined carrier quantum states in ultrathin semiconductor heterostructures," in Festkörper Probleme XV (Advances in Solid State Physics), H.J. Queisser, Ed. New York: Pergamon, 1975, pp.21-48.

[4] N. Holonyak, Jr., R. M. Kolbas, R. D. Dupuis, and P. D. Dapkus, "Quantum Well Heterostructure Lasers," IEEE J. Quantum Electron., QE-16, pp 170-186 (1980)

[5] D. A. B. Miller, D. S. Chemla, D. I. Eilenberger, P. W. Smith, A.C. Gossard, and W. T. Tsang, "Large roomi-temperature optical nonlinearity in $\mathrm{GaAs} / \mathrm{Ga}_{1-\mathrm{x}} \mathrm{Al}_{\mathrm{x}} \mathrm{As}$ multiple quantum well strucíures," Appl. Phys. Lett.. 41, pp 679-681 (1082)

[6] C. Weisbuch, R. Dingle, A. C. Gossard, and W. Weigmann, "Optical characterization of interface disorder in $\mathrm{GaAs}_{-} \mathrm{Ga}_{1-\mathrm{x}} \mathrm{Al}_{\mathrm{x}}$ As multi-quantum well structures," Solid State Commun., 38, pp 700-712 (1881)

[7] H. L. Störmer, R. Dingle, A. C. Gossard, W. Wiegmann, and R. A. Logan, "Eler': :onic properties of modulation-doped GaAs- $\mathrm{Al}_{\mathrm{X}} \mathrm{Ga}_{1-\mathrm{x}}$ As superlattices," Fourteenth International Conference on the Physics of Semiconductors, Edinburgh, 1978, B. L. H. Wilson, Editor, Inst. Phys. Conf. Ser. No. 43 (1979) pp 557-560

[8] H. C. Casey, Jr., M B. Panish, "Heterostructure lasers, Part B: Materials and operating characteristics," Academic Press, 1978; H. Kressel, J. K. Butler, "Semiconductor lasers and heterojunction LEDs," Academic Press, 1977 
[9] C. Lindström, R D. Burnham, and D. R. Scifres, "Visible ew single quantum well (AlGa)As diode lasers," Appl. Phys. Lett., 42, pp 134-136 (1983)

[10] J. O. Dimmock, "Introduction to the theory of exciton states in semiconductors," in "Semiconductors and and semimetals," Editors R. K. Willardson anci

A. C. Beer, Academic Press, 1967, pp. 259-320

[11] K. Cho, Ed., "Excitons," Springer-Verlag, 1979

[12] H. I. Ralph, "The electronic absorption edge in layer type crystals," Solid State Commum., 3, pp 303-306 (1865)

[13] R. C. Miller, D. A. Kleinman, O. Munteanu, and W. T. Tsang, "New transitions in the photoluminescence of GaAs quantum wells," Appl. Phys. Lett., 30, pp $1-3(1981)$

[14] R. C. Miller, D. A. Kleinman, W. A. Nordland, and A. C. Gossard, "Luminescence studies of optically pumped quantum wells in $\mathrm{GaAs}_{-} \mathrm{Al}_{\mathrm{x}} \mathrm{Ga}_{1-\mathrm{x}} \mathrm{As}$ multilayer structures," Phys. Rev. B, 22, pp 863-871 (1880)

[15] A. Yariv, "Quantum Electronics," John Wiley \& Sons, Inc., 1975, Chapter 8

[16] E. Burstein, A. Pinczuk, and D. L. Mills, "Inelastic light scattering by charge carrier excitations in two-dirnensional plasmas: theoretical considerations," Surface Sci. 08, pp 451-468 (1980)

[17] A. Pincruk and J. M. Worlock, "Light scattering tnechanisms in twodimensional electron systems in semiconductors," Surface Sci. 113, pp 69--84 (1982)

[18] E. Merzbacher, "Quantum Mechanics," John Wiley \& Sons, Inc., 1970, pp. 457-458

[19] J. D. Jackson, "Classical Flectrcdynamics," 2nd Ed., John Wiley \& Sons, (1975)

[20] E. O. Kane, "Band structure of Indium Antimonide," J. Phys. Chem. Solids 
1, pp 249-261 (1957); E. O. Kane, in "Semiconductors and Semimetals," Vol. 1, ed. Willardson and Beer, Academic Press, (1966)

[21] B. K. Ridley, "Quantum processes in semiconductors," Oxford University Press, (1982)

[22] J. S. Blakem" :e, "Semiconducting and other major properties of gallium arsenide," J. Appl. Phys. 53(10), pp. R123-R181, (Oct. 1982); and M. Nueberger, "IIl-V Semiconducting compounds," Handbook of Electonic Materials, Vol. 2, IFI/Plenum, New York, (1971)

[23] D. Arnold, A. Ketterson, T. Henderson, J. Klem, and H. Morkoc, "Determination of the valence-band discontinuity between $\mathrm{GaAs}$ and $(\mathrm{Al}, \mathrm{Ga}) \mathrm{As}$ by the use of $p^{+}-\mathrm{GaAs}-(\mathrm{Al}, \mathrm{Ga})$ As-p--GaAs capscitors," App. Phys. Lett. 45, pp. $1237-1239$ (1984)

[26] G. Bastard, "Superlattice band structure in the envelope function approximation," Phys. Kev. B. 24, pp. 5623-5607 (1881)

[25] T. Andc and S. Mori, "Effective-mass theory of semiconductor heterojunctions and superlattices," Surface Science 113, רo. 124-130 (1982)

[26] C. Kittel, "Introduction to solid state physics," Fourth Edition, John Wiley \& Sons, New York (1871)

[27] C. V. Shank, R. L. Fork, R. Yen, J. Shah, B. I. Greene, A. C. Gossard, and C. Weisbuch, "Picosecond dynamics of hot carrier relaxation in highly excited multi-quantum well structures," Solid State Comm. 47, pp. 981-983 (1983)

[28] Z. Y. Xu and C. L. Tang, "Picosecond relaxation of hot carriers in highly photoexcited bulk GaAs and GaAs-AlGaAs multiple quantum multiple quantum wells," Appl. Phys. Lett. 44, pp. 692-694 (1984)

[29] D. J. Erskine, A. J. Taylor, and C. L. Tang, "Femtosecond studies of intraband relaxation in GaAs, AlGaAs, and GaAs/AlGaAs multiple quantum well 
structures," Appl. Phys. Lett. 45, pp. 54-56 (1984); see also Erratum to this paper, Appl. Phys. Lett. 16, p. 614 (1985)

[30] D. J. Erskine, A. J. Taylor, and C. L. Tang, "Dynamic Burstein- Moss shift in GaAs and GaAs/AlGaAs multiple quantum well structures," Appl. Phys. Lett. 45, pp. 1209-1211 (1984)

[31] C. Weisbuch, R. Dingle, P. M. Petroff, A. C. Gossard, and W. Wiegmann, "Dependance of the structural and optical properties of $\mathrm{GaAs}_{\mathrm{A}} \mathrm{Ga}_{1-\mathrm{X}} \mathrm{Al}_{\mathrm{X}} \mathrm{As}$ multiquantum-well structures on growth temperature," Appl. Phys. Lett. 38, pp. 840-812 (109:)

[32] S. J. Allen, Jr., D. C. Tsui, and B. Vinter, "On the absorption of infrared radiation by electrons in semiconductor inversion layers," Solid State Comm. 20, pp 425-428, (1976)

[33] D. A. Dahl, and L. J. Sham, "Electrodynamics of quasi-two-dimensional electrons, ${ }^{n}$ Phys. Rev. B 16, pp. 651-661 (1977)

[34] W. P. Chen, Y. J. Chen, and E. Burstein, "The interface EM modes of a 'Surface Quantized' plasma layer on a semiconductor surface," Surface Science 58, pp. 263-265 (1976)

[35] P. M. Petroff, R. C. Miller, A. C. Goddard, and W. Wiegmann, "Impurity trapping, interface structure, and luminescence of GaAs quantum wells grown by molecular beam epitaxy," Appl. Phys. Lett. 44 pp. 217-219 (1884)

[36] W. T. Masselink, M. V. Klein, Y. L. Sun, Y. C. Chang, R. Fischer, T. J. Dramond, and H. Morkoç, "Improved GaAs/AlGaAs single quantum wells through the use of thin supperlattice buffers," Appl. Phys. Lett. 14 Fp. 435-437 (1984)

[37] F. Schäffler and F. Koch, "Subband spectroscopy at room temperature," Solid State Commun. 37, pp. 365-368 (1881) 
[38] H. Reisinger and F. Koch, "Spectroscopy of InAs subbands," Solid State Commun. 37, pp. 429-431 (1981)

[39] W. Beinvogl and J. F. Koch, "Spectroscopy of electron subband levels in an inversion layer on InSb," Solid State Commun. 24, pp. 687-680 (1977)

[40] J. Scholz, F. Koch, H. Maier, and J. Ziegler, "Coupled subband-phonon resonances in the far-infrared reflection from $\mathrm{Hg}_{1-\mathbf{x}} \mathrm{Cd}_{\mathbf{X}}$ Te surfaces," Solid State Commun. 45, pp. 30-42 (1983)

[41] P. Ruden and G. H. Döhler, "Electronic structure of semiconductors with doping superlattices," Phys. Rev. B 27 pp. 3538-3546 (1083)

[42] W. G. Spitzer, in Semiconductors and Semimetals, edited by R. K. Willardson and A. C. Beer, Academic Press, New York (1967) Vol. 3, pp. 43-48

[43] Martin A. Afromowitz, "Refractive index of $\mathrm{Ga}_{1-x} \mathrm{Al}_{\mathrm{x}} \mathrm{As}$," Solid State Comm. i5 pp. 59-63 (1974)

[44] B. O. Seraphin and H. E. Bennett, in Semiconductors and Semimetals, edited by R. K. Willardson and A. C. Beer, Academic Press, New York (1967) Vol. 3, pp. $490-543$

[45] Handbook of Chemistry and Physics, CRC Press, 63rd Edition (1982-1983)

[46] H. Morkoc, T. J. Drummond, and R. Fischer, "Interfacial properties of (A),Ga)As/GaAs structures: Effect of substrate temperature during growth by molecular beam epitaxy," J. Appl. Phys. 53, pp. 1030-1033 (1982)

[47] Jasprit Singh, K. K. Bajaj, and S. Chaudhuri, "Theory of photoluminescence line shape due to interfacial quality in quality quantum well structures," Appl. Phys. Lett. 44, pp. 805-807 (1984)

[48] James R. Chelikowsky and Marvin Cohen, “Nonlocal Pseudopotential calculations for the electronic structure of eleven diamond and zinc-blend semiconductors," Phys. Rev. B. 14, pp. 556-575 (1876) 
[49] F. M. Vorobkalo, K. D. Glinchuk, and V. F. Kovalenko, "Temperature dependence of the width of a direct forbidden band of $\mathrm{Al}_{\mathbf{X}} \mathrm{Ga}_{1-\mathrm{x}}$ As solid solutions," Sov. Phys. Semicond. 0, pp. 656-657 (1975)

[50] C. A. Chang, W. K. Chu, E. E. Mendez, L. L. Chang, and L. Esaki, "Molecular beam epitaxy of Ge-GaAs superlattices, ${ }^{n}$ J. Vac. Sci. Technol. 10, pp. 567-570 (1981)

[51] C. T. Seaton, S. D. Smith, F. A. P. Tooley, M. E. Prise, and M. R. Taghizadeh, "Realization of an InSb bistable device as an optical AND gate and its use to messure carrier recombination times," Appl. Phys. Lett. 42, pp. 131-133 (1883)

[52] D. A. B. Miller, D. S. Chemla, D. J. Eilenberger, P. W. Smith, A. C. Gossard, and W. T. Tsang, "Large room-temperature optical nonlinearity in GaAs/Ga $/ \mathrm{GAl}_{\mathbf{x}} \mathrm{As}$ multiple quantum well structures," Appl. Phys. Lett. 41, pp. 679-681 (1082)

[53] Optical computing is the subject of a special issue of the following two journals; Optical Engineering, 23, Jan./Feb. 1984 and Proceedings of the IEEE, 72, July 1984

[54] A. A. Sawchuk and T. C. Strand, "Digitsl optical computing," Proceedings of the IEEE, 723, p. 758 (1084)

[55] Alan Huang, "Parallel algorithims for optical digital computers," 10th International Optical Computing Conference, Cambridge, Mass., April 6-8, 1883.

[56] A. Szöke, V. Daneu, J. Goldhar, and N. A. Kurnit, "Bistable optical element and its applications," App. Phys. Lett. 15, pp. 376-379 (1969)

[57] H. M. Gibbs, S. L. McCall, T. N. C. Venkatesan, "Optical bistable devices: the basic components of all-optical systems?" Proceedings of the Society Photooptical Instrumentation Engineers, Integrated Optics, Vol. 269, pp. 75-80 (1981) 
[58] "Topical Meeting on Optical Bistability," June 15-17, 1983, University of Rochester, Rochester, New York, Optical Society of America, Vol. 83.8 (1983) [59] "Optical Bistability," Edited by C. M. Bowden, M. Ciftan, and H. R. Robl, Plenum Press, New York (1981)

[60| "Heat-transfer microstructures for integrated circuits," D. B. Tuckerman, Ph. D. thesis, Stanford University (1984)

[61] F. A. P. Tooley, S. D. Smith, and C. T. Seaton, "High gain signal amplification in an InSb transphasor at $77 \mathrm{~K}$," App. Phys. Lett. 13, pp. 807-809 (1083)

[62) G. L. Harnagel, T. L. Paoli, K. L. Thornton, R. D. Burnham, and D. I. Smith, "Accelerated aging of 100-mW cw multiple-stripe GaAlAs lasers grown by metalorganic chemical vapor deposition," App. Phys. Lett. 46, pp. 118-120 (1985)

[63] D. D. Coon and R. P. G. Karunasiri, "New mode of IR detection using quantum wells," Appl. Phys. Lett. 45. pp. 649-651 (1084)

[64] J. S. Smith, L. C. Chui, S. Margalit, A. Yariv, and A. Y. Cho, "A new mode infrared detector using electron emission from multiple quantum wells," J. Vac. Sci. Technol. B1, pp. 376-378 (1983) 
Appendix A: Evaluation of Coulomb Potential Integrals

\section{Part 1}

The first integral, $I_{1}$, is given by equation (IV.71) and repeated here as

$$
I_{1}=\int_{-\pi / 2}^{\pi / 2}\left[2 \theta^{2}-\cos (2 \theta)\right] \cos ^{2}(\theta) d \theta
$$

The evaluation of this integral is now illustrated. The integral is first separated into a sum of several integrals as follows.

$$
\begin{aligned}
l_{1} & =\int_{-\pi / 2}^{\pi / 2}\left[2 \theta^{2}-\cos (2 \theta)\right] \cos ^{2}(\theta) d \theta \\
& =\int_{-\pi / 2}^{\pi / 2}\left[\theta^{2}(1+\cos (\cdot-\cos (2 \theta)(1+\cos (2 \theta)) / 2] d \theta\right. \\
& \left.=\int_{-\pi / 2}^{\pi / 2}\left[\theta^{2}+\theta^{2} \cos (2 \theta)\right)-\frac{1}{2} \cos ^{2}(2 \theta)-\frac{1}{2} \cos (2 \theta)\right] d \theta
\end{aligned}
$$

The first integral over $\theta^{2}$ is trivially integrated with a result of $\pi^{3} / 12$. The second term is solved by integration by parts as

$$
\left.\int_{-\pi / 2}^{\pi / 2} \theta^{2} \cos (2 \theta)\right) d \theta=\left.\frac{\theta^{2}}{2} \sin (2 \theta)\right|_{-\pi / 2} ^{\pi / 2}-\int_{-\pi / 2}^{\pi / 2} \theta \sin 2 \theta d \theta
$$

The first term on the right hand side of equation (A.3) can be seen to be equal to zero. The second integral is again integrated by parts to obtain

$$
\begin{aligned}
-\int_{-\pi / 2}^{\pi / 2} \theta \sin 2 \theta d \theta & =\left.\frac{\theta}{2} \cos (2 \theta)\right|_{-\pi / 2} ^{\pi / 2}-\frac{1}{2} \int_{-\pi / 2}^{\pi / 2} \cos 2 \theta d \theta \\
& =\frac{\theta}{2} \cos (2 \theta)-\left.\frac{1}{4} \sin (2 \theta)\right|_{-\pi / 2} ^{\pi / 2} \\
& =-\frac{\pi}{2} .
\end{aligned}
$$

The third term can be integrated as directly with the result 


$$
\begin{aligned}
-\frac{1}{2} \int_{-\pi / 2}^{\pi / 2} \cos ^{2}(2 \theta) d \theta & =-\frac{1}{4} \int_{-\pi / 2}^{\pi / 2}(1+\cos (4 \theta)) d \theta \\
& =-\left.\frac{1}{4}\left(\theta-\frac{1}{4} \sin (4 \theta)\right)\right|_{-\pi / 2} ^{\pi / 2} \\
& =-\frac{\pi}{4} .
\end{aligned}
$$

The last term is easily seen to integrate to zero. The integral $I_{1}$ in equation (A.1) can now be evaluated with the use of the above equations to find

$$
I_{1}=\left[\frac{\pi^{3}}{12}-\frac{\pi}{2}-\frac{\pi}{4}\right]=\left[\frac{\pi^{3}}{12}-\frac{3 \pi}{4}\right]
$$

\section{Part 2}

The second integral, $I_{2}$, is given by equation (IV.74) and repeated here as

$$
\begin{aligned}
I_{2} & =\int_{-\pi / 2}^{\pi / 2}\left[2 \theta^{2}-\cos (2 \theta)\right] \sin ^{2}(2 \theta) d \theta \\
& =\int_{-\pi / 2}^{\pi / 2}\left[\theta^{2}(1-\cos (4 \theta))-\sin ^{2}(2 \theta) \cos (2 \theta)\right] d \theta .
\end{aligned}
$$

The second term in the integral is evaluated by parts as

$$
\begin{aligned}
\int_{-\pi / 2}^{\pi / 2} \theta^{2} \cos (4 \theta) d \theta & =\left.\frac{\theta^{2}}{4} \sin (4 \theta)\right|_{-\pi / 2} ^{\pi / 2}-\frac{1}{2} \int_{-\pi / 2}^{\pi / 2} \theta \sin (4 \theta) d \theta \\
& =\left.\frac{\theta}{8} \cos (4 \theta)\right|_{-\pi / 2} ^{\pi / 2}-\frac{1}{8} \int_{-\pi / 2}^{\pi / 2} \cos (4 \theta) d \theta \\
& =\frac{0}{8} \cos (4 \theta)-\left.\frac{1}{32} \sin (4 \theta)\right|_{-\pi / 2} ^{\pi / 2} \\
& =\frac{\pi}{8}
\end{aligned}
$$

The last integral in equation (A.7) is easily integrated as

$$
\int_{-\pi / 2}^{\pi / 2} \sin ^{2}(2 \theta) \cos (2 \theta) d \theta=\left.\frac{1}{6} \sin ^{3}(2 \theta)\right|_{-\pi / 2} ^{\pi / 2}=0
$$


The integral $I_{2}$ is now found with the use of the above equations to have the value

$$
I_{2}=\left[\frac{\pi^{3}}{12}-\frac{\pi}{8}\right]
$$


Appendix B: Design of an Optical Digital Computer

The primary motivation for an all optical digital computer is enhanced computational power. Secondary benefits of reduced power and simpler fabrication over electronic approaches also appear possible. The advantages of optical logic primarily lie in the elimination of inductance-resistance- capacitance problems inberent in electronics. These problems often become the limit in the speed of an electronic system. In addition, the relatively slow speed of an electron across a gate, which can slow down an electronic logic element, requires very small lithographic regions to minimize this time. An optical gate does not have this problem and can be much larger for the same speed. Also, the interconnect is much easier to build optically than electrically at Terahertz frequencies.

\section{The approach: lenses vs. waveguides}

The approach to optical computing described here differo fundamentally from the more common approaches to optical computing under current investigation. The optical logic described here is designed for use in a system interconnected by integrated optical waveguides. In contrast, the more common research direction in optical computing ${ }^{[53]}$ is based on the free space propagation of optical signals, with processing being performed in the image or fourier planes of an optical imaging system. In these imaging systems, lenses and other optical elements are used as conduits for information in an array (1000 by 1000 or so) transverse to the optical direction of propogation. Examples of optical systems of this type, which also show promise for performing the full set of logical operations neccasary for computing, are given by the works of Huang[54] and Sawchuck. [55]

The two types of computer systems, which differ on the type of interconnection used, both have significant advantages depending on the type of computing which must be performed. An optical computer interconnected by free space propagation 
is limited to a logic array in two dimensions since the third axis is used for transit of optical signals. Because a given logic element always has a minimum size, this limit to two dimensions severely limits the number of logic elements which can be placed within a given communication time of each other. In contrast, the system based on integrated optics can stack many logic boards, created in two dimensions by standard lithographic techniques, in a third dimension in a very similar manner to a current electronic computer. This difference is very important for a serial processor, with its speed limited by the time of transit of the signals between a large number of logic elements. But the difference is not significant for a parallel processor which does not rely on the speed of a given operation as much as the ability to perform operations simultanecusly with a large number of logic elements at a very low cost per element. The integrated optical interconnect is thus seen to be advantageous for very fast serial processors and the free space interconnect advantageous for low cost parallel processors.

The choice of interconnect has implications for the optical wavelength, the type of nonlinear media used in the logic element and the design of the logic gate itself. The wavelength of the light used for the integrated optical elements should allow fabrication of optical waveguides and integrated optical elements, to be described later, with simple lithographic techniques. For media with an index of refraction of 3 and lithographic resoiution of about 1 micron, the wavelength of the light should be about 10 microns. The wavelength of light for a free space interconnect system should be as small as possible since the resultant reduction in the resolvable pixel size reduces the optical power requirements and increases the logic element density on the surface of the array. The availability of high quality and inexpensive visible lenses and imaging system give strong preference to this optical band for free space systems. Futher differences in the design of the two systems are in the geometry of 
the logic element. A logic element the free space should have all inputs arriving from the axis of signal propogation whereas the integrated optical logic element should have all inputs arriving from within the plane of the given optical logic board in which it lies. This obvious condition complicates use of the QWEST in a free space system since its dipole is zero for all light incident perpendicular to the growth plane. Furthermore, a free space system is intrinsically slower than an integrated optical system because of the lower density of optical logic elements and the longer transit times between them. Thus a slower nonlinearity with a lower optical power requirement would be useful, suggeating the QWEST is not optimal for this type of system.

The choice to develon integrated optical systems over free space systems was made for several reasons. First, not all computations of interesi can be properly partitioned to be solved by a parallel processor. Second, if a parallel processor is desired, then it can be obtained by connecting together several serial processors. But the reverse approach is not possible, an intrinsically parallel machine makes a very poor serial processor. The fabrication of integrated optical elements is much more difficult than free space elements, but once the hardware is deve.uped, a much superior system should be achieved. Furthermare, although the fabrication costs of a given logic element in a free space system are lower than a logic element in an integrated optical system, the lower speed of the free space system requires proportionally more logic elements in parallel to acheive the same throighput as an integrated optical system. For comparison, a free space system consisting of a $1 \mathrm{~mm}$ square logic array connected by a series of lenses with a maximum optical path length of $1 \mathrm{~cm}$ will create a system compatable with a logic gate speed of under .00 picoseconds. An integrated optical computer consisting of a stack of 20 boards in a $1 \mathrm{~mm}$ cube can take full advantage of the speed of a logic element 
with a speed under 1 picosecond. This factor of 100 in speed ratio between the two systems requires the free space system have a cost, when divided by the number of logic elements, a factor of 100 below that of the integrated optical system simply to be comparable for processes capable of being effectively partitioned in a parallel processor. Unfortunately, the economics of these systems is unknown with any precision until further development. However, the fast serial processor is slearly only achievable by the integrated optical interconnect, and is clearly needed for some applications, independent of the performance of parallel systems. Research on this type of processor is justified for this reason alone. Furthermore, present analysis indicates that an integrated optical digital computer to be described is capable of improving the speed and lowering the power used both by a factor of 1000 over current electronic computers. This improvement combined with fabrication by current semiconductor industry methods suggests that ihis technology may also find widespread utility within the computing demands of the future.

\section{The requirements of an optical logic element}

A digital computer consists of logic elements, their interconnection, and memory, all supprited by a framework consisting of a power supply, cooling and connection to the outside world. A properly working computer demands $r$ ance and proper proportion among these components. But the speed and performance of a computer depends primarily on the logic element, not orly because of its speed but its functionality and characteristics. Ten requirements for an optical digital logic eleinent are now given which are essential to a working computer system. 

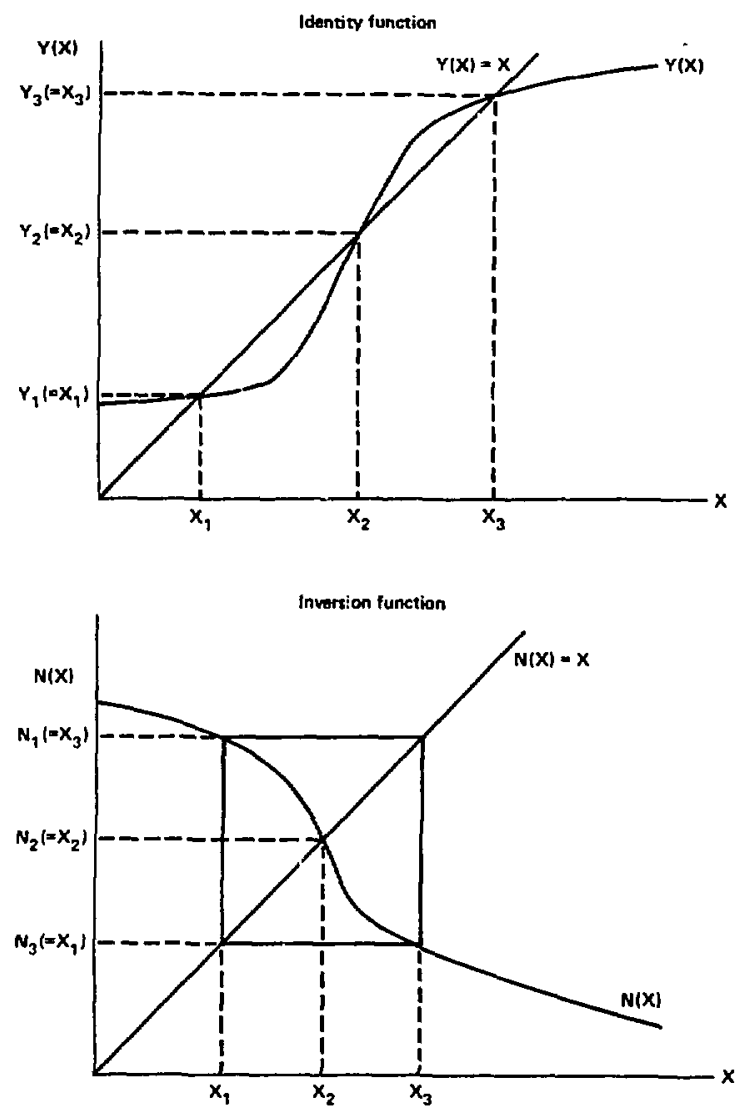

Figure B.1: The desired transfer functions of a logical identity and inverter operation.

The first requirement of a logic element is an output power as a function of the input power, or transfer function, with a proper form. Because the power of the optical signal varies significantly with location, the input is considered for purposes here to be the value of output of the previous logic element. This definition allows the transfer function to include the effects of the interconnection and beamsplitting. 
If we momentarily take a logic element with only ore $\quad t$, then this element will be either an identity or inversion operation. The tra sicr function of the identity operation must cross the output=input axis tbree times (see Fig. B.1). The lowest power point is a "low" signal and the highest power point is a "high" signal. Both the upper and lower power points must have a slope less than unity at the crossing point in order to be stable. The intermediate power point must have a slope greater than unity. A similar argument also applies to the inversion operation. The transfer function should have very little hysteresis and be single valued. The "low" power value should be sufficiently small so that several low inputs to a logic element do not combine to appear as a "high" and change the output of the gate.

The second requirement is for gair of the signals from the input of a civen logic element to its output. This gain is needed to allow sufficient power to drive many other logic elements and allow for losses in the interconnects. Gain should be a factor in the range of 4 to 10.

The third requirement is to prevent random phase variations in the optical signals from affecting the function of the logic element. This requirement is unique to optical waves and has no parallel for electronic computers. The problem arises from the wave nature of light and the phase relationship among the signal inputs and the light power source. These waves must be present in the same physical location since they must interact through the same dielectric. But the effect of these waves on the media depends on the local intensity, which varies radically depending on the relav:re phase of the interacting light signals, even if their individual intensities remain constant. Furthermore, the signals arrive from different locations through long waveguides, which can change the phase of the light at a logic element via small changes in the refractive index. The great difficulties in predicting and and maintaining a precise phase relationship between all beams indicates that the logic 
element should be designed to be independant of the phase relationship of the interacting sources.

The fourth requirement is the provision of the complete logic family. In particular, an inverter operation is mandatory. In addition the OR or AND logic operation is also needed to provide the complete logic set. A very powerful logic element is the complementary NOR or NAND logic elements. These logic elements provide the $\mathrm{OR}$ or AND operation and its inverse simultaneously. This duality has many advantages. It can reduce the overall computer optical power requirements, logic element count, and number of gale delays per operation all by as much as a. factor of 2. Furthermore, because an OR operation will turn into an AND operation upon inversion of all inputs and outputs, it entirely eliminates the need for bollh operations, thus the complete logic family can be accomplished with only one logic element. For these reasons, a complimentary NOR or NAND logic operation is strongly preferred.

The fifth requirement is for a logic element which does not reflect the input signals (or be "impedence matched") for all possible states in which the logic element may exist. Reflection of the inputs can obviously affect the performance of the previous logic element and also can set up cavity resonancss between the output of one element and the input of the next. These resonances depend critically on the length of the transmission line and can also affect the transmission efficiency randomly.

A sixth requirement for the logic element is for multiple inputs. Although only two inputs are needed to create the entire logic family, actual computers can use many more with such great freguency that a gate with only two inputs could slow today's computers by a factor of over 2 , while again doubling the logic gate count and overall power requirements. As such, a logic gate with at least 4 inputs is 
generally preferred. Again recalling requirements three and five, these inputs should not interact with each other, except as needed for the logic operation, and not be reflected for any logic state.

A seventh requirement for a logic element is the tolerence to variations in manufacture (dopant density, linewidth, etc. ) and environmental conditions (temperature changes and gradients, dust, etc. ). A it gic element which does not have a transfer function or other properties which are tolerent to these variations will fail. For instance, the temperature of a logic element could easily ry by more than $10^{\circ} \mathrm{C}$, yet this temperature change will cause the index of refraction of GaAs to change by $1.5 \times 10^{-3}$ and the bandgap of GaAs by $5 \mathrm{meV}$. Neverthe' ss, many designs are seen which are sensitive to an order of magnitude smaller variations with predictable results.

An eighth requirement is for the logic element to be manufacturable The manufacture of the logic element (and its interconnections) should be suffic sntly inexpensive to be competitive with electronic logic and fabricatable in very large numbers with reasonable reliability and yields.

A ninth requirement is for low output noise on the signal lines. Most optical logic tends to have low noise simply because of the very high frequency of the carrier signals and low numbers of optical modes involved. However, narrow bandwidth sensitivity and isolation of crosstalk will reduce this noise margin even further.

The tenth requirement is that all obvious conditions be satisfied. For instance, a logic family would not be able to make \&n optical computer if the output wavelengths did not match the required input wavelengths. Many other conditions of this type exist and must also be satisfied. 


\section{Examples of an optical logic element}

In a logic gate, the optical beams will interact through a nonlinear material. This interaction will be described by the dielectric constant of the material. The optical beams will have an effect on this dielectric constant and this dielectric constant will in turn describe the effect of the nonlinear material on the optical beams. The higher the nonlinearity of this material, the lower the optical power needed to create a given effect. The logic element can use either the refractive or absorptive part of the dielectric constant and often is a combination of both.

A cornmonly used component of an optical logic element is a Fabry-Perot resonator formed by two mirrors with a nonlinear material in between. The frequency of the optical signals is tuned to be slightly off resonance of the nonlinear material so as be more affected by the change in refractive index rather than the change in absorption. The cavity will be resonant only when an integer number of half wavelengths is equal to the cavity length. But since this wavelength depends on the refractive index, and therefore the optical power within the cavity, the cavity will tend to transmit only a particular power, no matter what power is incident. Although if the incident optical power should drop too low the cavity will simply switch to no transmission. The behavior of these nonlinear cavities is described in several references. ${ }^{[50-50 \mid}$ A simple example ${ }^{[01]}$ of the form of the transfer function of this cavity is shown in Fig. B.2. The cavity can be biased so that the resonant phase occurs for any particular optical power for the first transmission step. However, all further cavity resonances must have sufficient power to shift the rond trip phase of the cavity by multiples of $2 \pi$. Because the power of a nonlinear element must be kept at a minimum, a logic element will attempt to work with the first cavity resonance biased to occur with very little incident power. How small this bias can be made is dependent on the properties of the Fabry-Perot cavity. A cavity, 
formed by mirrors with transmission $\mathbf{T}$ and single pass loss in optical intensity lower than a factor of $T$, will have an internal optical intensity increased by a factor of $1 / T$ over that incident when in resonance. This cavity will not significantly change its transmission factor until the optical phase has been shifted by an amount of order $\pi / T$ from the cavity resonance. This cavity property sets the minimam phrise bias which is usable and therfore the mimimum optical power of an optical elemeni. The limits on this resonant enhancement are set by such factors as the buildability of the high reflectivity mirrors, a sufficiently low unsaturable loss in the cavity, and uitimately, the optical cavity decay times, which rapidly become significant, for high reflectivities and large cavities. Typical cavities used will have lengths of order 1 to 10 microns with mirroris of transmission between 1 to $10 \%$. The cavity decay times, for materials with a refactive index of 3 , will be of order .1 to 10 picoseconds.

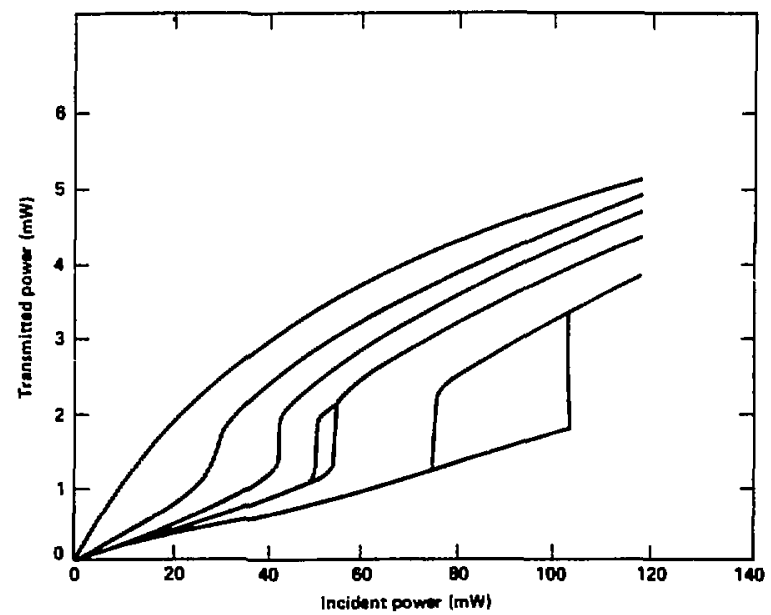

Figure B.2 An example of a transfer function of an optical cavity containing a nonlinear material (From Ref. 61).

A nonlinear Fabry-Perot cavity does not in itself create a logic element which satisfies the previous requirements. An example is now presented which uses the 
Fabry-Perot cavity, the QWEST and integrated optics to satisfy the earlier requirements. The fabricated structure is illustrated in Fig. B.3. This structure contains a number of features which aid in the physical behavior and manufacturability of the device. The integrated optical structure consists of the materials Ge, GaAs, AlAs, and $\mathrm{ZnSe}$ which are not only of similar lattice structure but have nearly identical lattice sizes. Furthermore, they have all been demonstrated to grow well upon one another epitaxially as a single crystal. The refractive index of these materials at 10 micron wavelengths is $4.0,3.3,2.8$, and 2.4 respectively. These materials are all very transparant for light with wavelengths from 2 microns (limited by the Ge bandgap) to well beyond 10 microns (limited by phonon absorption in several of the materials). Epitaxial growth of various low index (1.4) materials such as $\mathrm{BaF}_{2}$ and $\mathrm{CaF}_{2}$ has been demonstated on GaAs and Ge substrates with sufficient quality to be a good cover material for these logic elements aud waveguides. A large index difference between the cover and waveguide material can be used to allow efficient control of the signals, as exhibited for instance in the right angle corners and waveguide cross-over (see Fig. B.3). Becalise of the ease of growtb of Ge and the (AlGa)As compounds (which has even lead to alternating $\mathrm{GaAs}$ and $\mathrm{Ge}$ quantum wells $\left.{ }^{(50}{ }^{5}\right)$, the less common $\mathrm{ZnSe}$ growth could be substituted entirely by waveguides made of $\mathrm{Al}_{\mathrm{x}} \mathrm{Ga}_{1-\mathrm{x}}$ As with $\mathrm{x}$ being close to unity. 

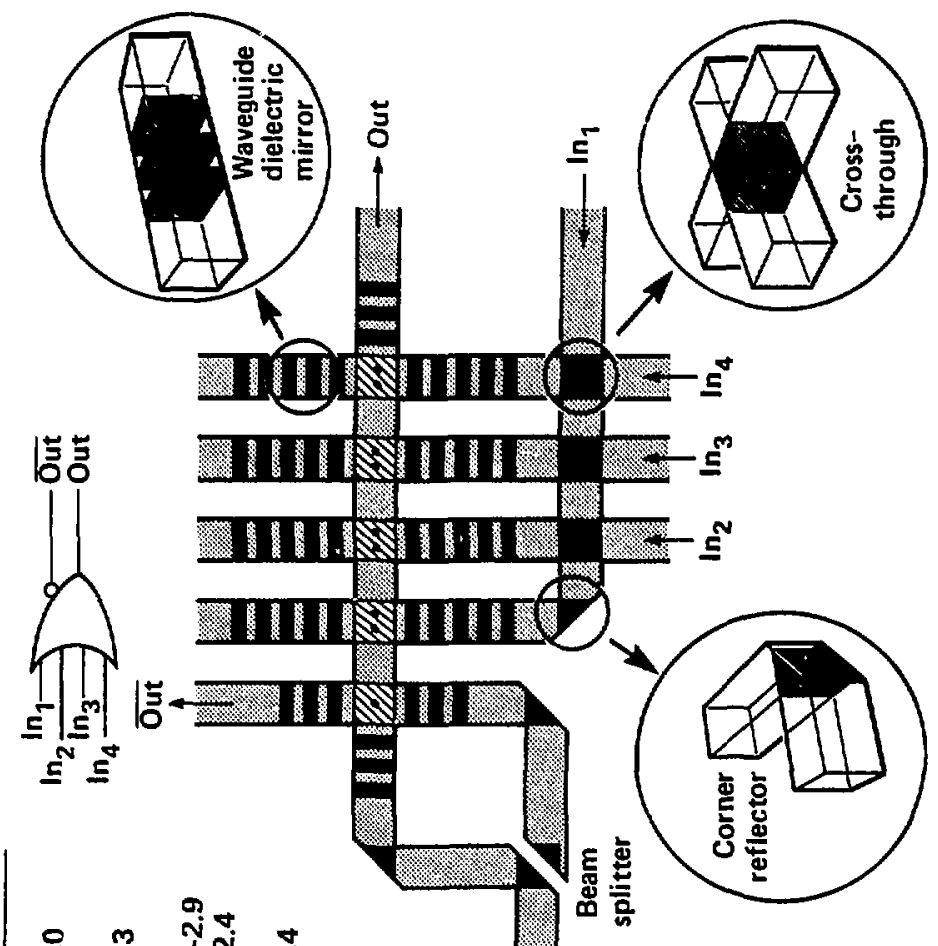

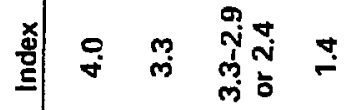

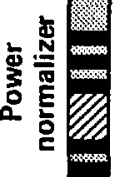

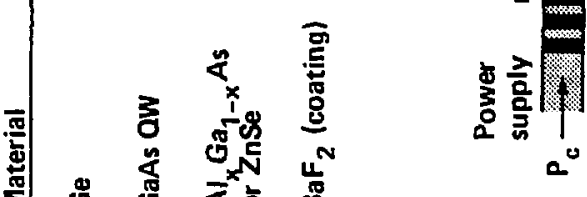

Figure B.3: An example of a complementary NOR optical logic gate, also illustrating several interconnection elements such as right angle corners and crossthroughs. 
There are several new integrated optical elements illustrated in Fig. B.3. One of the more important is the waveguide dielectric mirror. This mirror is an analogue of the dielectric mirrors used with lasers today. This mirror differs from a distributed feedback integrated optical mirror in that the alternating dielectric material extends fully across the waveguide instead of merely being a surface perturbation. This device is much easier to fabricate with today's technology for use in the infrared region than with visible light. A mirror with 90 to $90 \%$ reflection can be made with 3 to 5 quarter wave thick Ge slabs inserted into an AlAs wavequide. A second very important element is the corner reflector. The large refractive index of $\mathrm{Ge}$ and the small refractive index of the coating material allows the waveguides to make very abrupt bends, important for minimizing the space needed for interconnection. An abrupt waveguide bend is illustrated in Fig. B.3 which uses total internal reflection in a Ge prism to turn the light. Note that this prism does not appear to directly reflect rays from the center of one waveguide into the other. This is a result of the Goos-Hänchen shift, which makes the effective reflection surface appear behind the actual surface. The waveguide as shown is fairly inefficient, with losses of over 20 $\%$ to diffraction away from the corner. However, more complicated designs may reduce this loss to under $5 \%$. Yet another important integrated optical element is the cross-through, also illustrated in Fig. B.3. This element allows two waveguides to cross through one another without any exchange of signals between them. This element allows the signals to remain in one plane if so desired. The reduction in signal planes greatly aids in fabrication and compactness of optical computer systems. The cross-through allows the waves to be continually confined as they transit through the higher index Ge block. The reflection off of each surface is about $3 \%$ for the indices of refraction involved, but if the waveguides are made close to a half integer number of wavelengths wide, then the Ge block will be a 
resonant cavity and allow the optical signals through without any reflection loss. Also illustrated in Fig. B.3 is a beam splitter based on the prism corner reiector. The beam splitter has an additional corner which catches the infrared radiation which is exponentially decaying away from the reflection surface. For the materials indicated in Fig. B.3 and 10 micron wavelengths, this gap will be about 1.5 microns for a $50 \%$ coupling beamsplitter.

With this understanding of the integrated optical elements and the nonlinear Fabry-Perot cavity, the operation of logic element presented in Fig. B.3, a complementary NOR gate, can be explained. The infrared power to this logic element is supplied externally ( and quite possibly external to the computer in the form of bulk laser such as a $\mathrm{CO}$ or $\mathrm{CO}_{2}$ ) and applied to a power limiter. This power limiter is a nonlinear Fabry-Perot cavity biased to be resonant for the exact infrared power needed for the logic element. This limiter allows the external power to fluctuate from continous to Terahertz frequency with up to $50 \%$ variation while still providing a constant output with only a few percent variation. The limiter operates by a similar mechanism used in the logic element. As such, the output is similarly dependent on such parameters as tavity length and quantum well doping density as the logic element. Thus the limiter also reduces requirements on the tolerances of these parameters to a m! !ch easier relation between its cavity and that of the logic element a few microns away on the same substrate. After the power limiler, the beam splitter is used to properly proportion the infrared intensity between the two cross coupled logic cavities. The accuracy of this split is essential and must be within about $3 \%$ for logic cavities with a $10 \%$ transmission mirrors. This accuracy implies a beamsplitter gap tolerance of under 0.1 microns, which may be excessively difficult. The power limiter would then best be placed on each of the infrared paths after the beamsplitter as close as possible to the logic cavities instead of its present 
location.

The logic element consists of two Fabry-Perot cavities cross-coupled to one another, with one cavity greatly extended to provide areas for the inputs to couple. These logic cavities are biased such that, with as logic signal input, the shorter cavity transmits the infrared intensity incident and the longer cavity is close to resonance, but does not quite have sufficient infrared power incident to turn on. The infrared power incident on the longer cavity is greater than that incident on the shorter cavity. This greater power is needed because of the larger number of nonlinear abs cption regions in this cavity and the desire for this cavity, when on, to turn off the shorter cavity by driving it off resonance. The longer cavity is biased off resonance by a much larger factor than the smaller cavity because of the large number of nonlinear regions in this cavity. The input signals to the logic element. saturate the nonlinear regions and cause the longer cavity to draw photons into it by both lowering the loss of this cavity and pulling it closer to resonance. The high intensity infrared field in the longer cavity shifts the cavity resonance of the smaller cavity sufficiently far off to not allow any transmission. Thus a sufficiently strong infrared signal on any of the input lines causes the longer cavity to change its output from a low intensit: ' o a high intensity, or the OR operation, and the shorter cavity output from a low intensity to a high intensity, which is the NOR operation. The gain is given by the ratio of the output infrared intensity to the input signal required to switch it. The gain is directly proportional to the fan-out, or the number of logic elements a given ontput can drive. The gain is given by the ratio of the logic cavity transmission to the input cavity transmission. A large ratio indicates that the relatively smaller intensity of the signal builds up to a larger intensity inside the logic cavity and thus control it. Yet other factors relating to doping density and overall logic cavity absorption must also be satisfied to create 
a good transfer function with high gain. However, the gain of the logic element shown is in the range of 5 to 10 .

The nonlinear interactions occur only at the antinodes of these cavities. A change in the dielectric constant at the node of the cavity has no effect on either the cavity resonance or absorption loss. Furthermore, the excitation of these antinodes is much higher than that of the nodes simply because of the greater infrared intensity at these points. The nonlinear interaction is therefore considered not to take place homogeneously, but rather at these cavity antinodes which are indicated in Fig. B.3 by black spots. A cavity with mirrors of transmission $\mathrm{T}$ requires a change in phase of about $2 \mathrm{~T}$ to change the cavity from transmitting to blocking. We label the phase change required as $\Phi_{0}$. For a mirror transmission of $10 \%$, this phase change using the QWEST can be acheived with a power of about 2 milliwatts. However, several of these interaction regions, each of which are capable of this large a phase change, are seen to be required to create an operating logic element. These requirements, combined with the power losses in the limiter, lead to an overall logic gate power of about 50 milliwatts. The speed of this element is primarily limited by the approximately 3 to 5 picosecond time for the electron gas in the quantum welis to relax back to lattice temperature. Additionally, the longer logic cavity requires about 3 picoseconds to discharge its photons. Thus this logic eiement is anticipated to have a switch speed of under 10 picoseconds.

The inputs to the logic gaice can have an arbitrary phase relation $w$.th the other inputs and the power source since the relative phase depends on the lovation of the original source, its phase and the net phase shift after transversing a long path, all of which are difficult to predict and fluctuate with time and temperature. The logic gate is made insensitive to this phase relation by several means. The inputs are seperated physically from one another such that thier phase relation is not directly 
significant. The phase relation between the inputs and the cavity fields directly affects the local infrared intensity at the nonlinear interaction points. This relation is compensated by allowing the input, which is a single transverse waveguide mode, to interact with two adjacent cavity antinodes. The adjacent antinodes are $180^{\circ}$ out of phase with each other but the input signal does not change phase. If we let $x$ be the cavity field at one antinode and $-x$ the field at the other, then the effect of a signal with field $y$ at both antinodes is proportional so the sum of the intensities at the two antinodes. This effect is thus proportional to $(x+y)(x+y)^{*}+(x-y)(x-y)^{*}=2 x x^{*}+2 y y^{*}$ and phase relational $x y^{\star}$ part drops out.

This logic element based on the QWEST is also relatively independent to changes in temperature. The QWEST resonance has been measured to change by less than $0.16 \mathrm{meV}$ for a $10^{\circ} \mathrm{C}$ change in temperature. For a signal biased about $7 \mathrm{meV}$ away from resonaner this small change is negligable. The ionlinear effect of the QWEST can also be affected by a change in the decay or dephasing time with temperature. However, these quantities also appear to change very little over broad temperature ranges.

The quantum well doping densities needed for the logic device of Fig. B.3 are in the range of 4 to $20 \times 10^{18} / \mathrm{cm}^{3}$. For proper operation of the logic element, this doping density should be accurate to about $3 \%$. The presence of the power limiter can reduce this accuracy requirement by a factor of at least 3 to over $10 \%$. The limiter also reduces a requirement for a cavity length accuracy to 0.1 micron to a much easier requirement that the lengths of the limiter and logic element cavities be equal to within 0.1 microns.

The largest fault of the logic element of Fig. B.3 is the lack of good impedence matching of inputs to the logic element. is the optical intensity of the logic cavities change, the reflectivities of the input cavities (used to assure high absorption 
efficiency of the signals) change the reflected infrared signal intensity. A proper impedence match in this system is easiest obtained be placing a large waveguide attenuation in the path of the signal. This reduction in reflected signal also has the unfortunate consequence of requiring more power to the input and reducing the gain and fanout available.

This logic element is felt to be capable of demonstrating all the propertins needed for an optical logic element, but is certainly not an optimal device. The value of this device is considered to be proof of principle ( or a counterexample to the impossibility proofs ) in a readily nanufacturable device. The relatively high powe: of this logic gate is compatable with a room temperature computer with maximum dimensions of about 3 centimeters, cooled by demonstrated techniques. Suprisingly, a computer based on this germinal logic element could itself be a significant advance over current computational capabilities at a very reasonable cost. However, logic elements of this type are nevertheless considered an intermediate demonstration for yet much better performance devices to be designed.

\section{$\underline{\text { Logic element improvements }}$}

The above coupling mechanisms were enhanced by an electromagnetic resonatine of the Fabry-Porot cavity, which aided in lowering the power requirements by a factor equal to the mirror transmission. This resonance has its linits, both because of the difficulty in obtaining a cavity with a loss lower than the mirror transmission and in the lifetime of the cavity, which increases as the inverse of the mirror transmission. These considerations limit use of this enhancement to mirror transmissions in the range of 0.01 and higher, for a maximum performance increase in the range of a factor of $\mathbf{1 0 0}$. However, improvements in fabrication tochnique could eventually increase this electromagnetic enhancement by another order of magnitude. 
But other enhancements of the electromagnetic field are possible. One of the more interesting occurs in crystals much smaller than the wavelength of incident light. These small crystals have resonances for various negative ratios of the dielectric constant of the crystal with its surroundings, $\epsilon_{r}$. For instance, the electric field inside a dielectric sphere, $E_{i}$, in a uniform field, $E_{0}$, is also uniform and given by $E_{i}=3 E_{0} /\left(\epsilon_{r}+2\right)$. A dielectric constant can be negative by several mechanisms. The most common occurs when the light oscillates below ihe plasma frequency of a metal, semimetal, or highly doped semiconductor. Another frequency band with a negative dielectric constant occurs in between the longitudal and transverse optical phonon frequencies of an crystal, typically in the mid-infrared. The infrared wavelengths of interest are generally too small to be near the plasma frequencies of metals and too large to allow use of doped semiconductors. The semimetal of most interest is the As crystal, which bas an electron density in the range of $10^{20} / \mathrm{cm}^{3}$. The electomagnctic resonance can be obtained by adjusting the shape of the crystal, the dielectric constant of the surrounding material, the applied infrared frequency, and possibly the electon concentration of the plasma.

The resonances of these small crystals only depend on the shape of the crystal, but not the size if they are much smaller than the wavelength of incident light. The small size of these crystals gives nearly instantaneous response, even for very large enhancements, unlike the Fabry-Perot resonance. The largest problem with the use of these crystals is the large Rayleigh scattering which also resonates with the enhancement of the crystal fields. Rayleigh scattering decreases with increasing wavelength to the fourth power and decreasing scatterer size to the sixth power. Thus, with infrared wavelengths this resonance scattering may be elimimated with particles of dimensions less than about $\mathbf{3 0}$ nanometers. 
If the Rayleigh scattering of these crystals can be eliminated, then their application to a logic device can be made simply by their implantation at the interaction antinodes of the previous logic element. The crystals lower the infrared power needed for the logic element by confining optical fields into small volumes of nonlinear materia! with the same excited state density required to create a given change in refractive index. Because of the difficulties of manufacturing these small crystals with good uniformity, new logic designs are being investigated which allow the scattering to occur. Two possible scattering-coupled integrated optical interferometer designs are illustrated in Fig. B.4. The logical operation of these devices depends on the scattered radiation being collected with good efficiency in another mode of the cavity. The switching can result from, as before, a change in refractive index of the coupled modes in a Fabry-Perot cavity, or more likely, from a change in the scattering coefficient itself as a result of the local infrared intensity. This local intensity in turn depends on the scattering-coupled modes and provide a reedback mechanism. The sigual inputs can then change this talance and cause a switching of the modes with gain. These interferometers need to use both first and second order waveguide modes at each of the four waveguides, for a total of eight interaction channels overall, in order to allow sufficient numbers of connections to the external elements. Other electromagnetic enbancements also exist, but are more difficult to use. All of thrse enhancements can theoretically be used in conjuction with one another and with the improvements multiplying. 

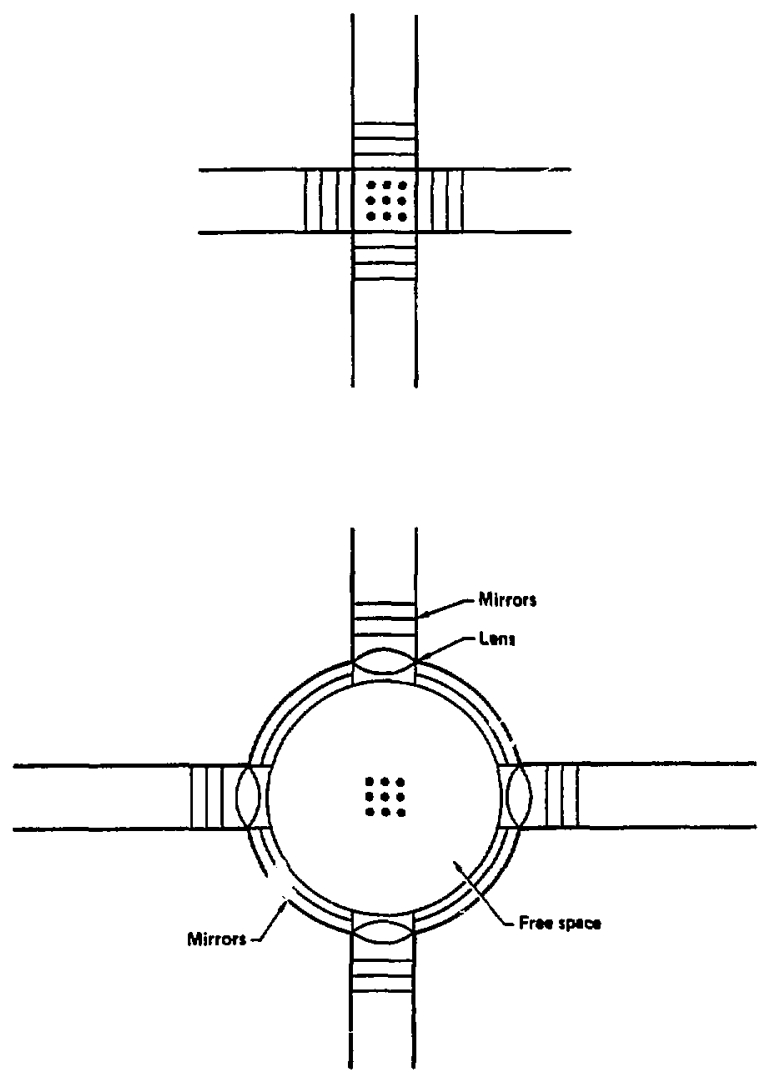

Figure B.4: An example of a scattering-coupled interferometer.

The limits to the enhancements are quickly approached from quantum noise statistics. If we allow a logic signal to be represented by only 300 quanta, and let these photon quanta have an energy as low as $0.1 \mathrm{eV}$ (or voltages of .1 volt for electronic charge quanta), then the power in a picosecond signal is equal to 5 microwatts. For an average gain of 3 (although some elements will have much higher gain) the average power to the logic elements must be about 3 times higher or about $\mathbf{1 5}$ microwatts. It can be seen that we only use a factor of about $\mathbf{3 0 0 0}$ in enhancements before we run into significant noise problems. Use of higher 
energy photons ( or higher voltages for electronic logic) only increases the energy requirements proportionally. Incidentally, the power-time product of such a low power logic element would be about 0.015 femtojoules, and appears quite possible if proper logic function can be found utilizing these electromagnetic enhancements in a fabricatable element.

\section{Optical logic element packaging}

The optical logic element of Fig. B.3 must be placed into a computer system to operate properly. We first make the assumption that the logic element has a switch time of 10 picoseconds. This time is predicted by measurements of the relaxation time of the electron temperature to the lattice temperature and the decay time of the logic cavity. The computer is taken to be a size, by comparison with today's supercomputers, which allows the signals to propagate across in about 15 logic gate delays. If the waveguides have an effective index of refraction of 1.5 , then the computer can be about 3 centimeters in maximum dimension. A possible physical structure of the computer is illustrated in Fig. B.5. This structure consists of 100 boards, each of which is 300 micrometess thick and 3 centimeters square. The overall computer is anticipated to contain approximately 1 million logic structures, with 10,000 on each board, and an average distance between logic elements of about 300 microns. Each board will consist of many chips, each with the most number of logic elements compatable with high yield and reliable fabrication. If we assume about 100 logic gates per $c^{\prime} ;$ ' The signals are routed within the chip on the same plane uning cross-through devices shown in Fig. B.3. Tha signals couple to each other through a backplane directly above the chips and onsisting of low index (1.5) waveguides. The chips couple the the backplane optically via a grating stiucture which produces a 50 micron diameter infrared beam perpendicular to the surface. A chip with 100 logic elements 
would possibly have as many as $\mathbf{3 0}$ connections. This $\mathbf{5 0}$ micron infrared spot size greatly eases tolerances to dust and translation errors, and could be increased further if needed. An infrared beam of this diameter can propogate over 700 microns without diffracting, so a 50 micron gap between the signal backplane and the chips is allowed without change in overall system function. Board to board connection is accomplished by another backplane at the edges of the logic boards. Electronic computer backplanes connecting similar densites of chips with multilevel printed circuit boards will have between 6 and 12 levels. The ability to cross waveguides through one another is expected to reduce this number of signal planes significantly.

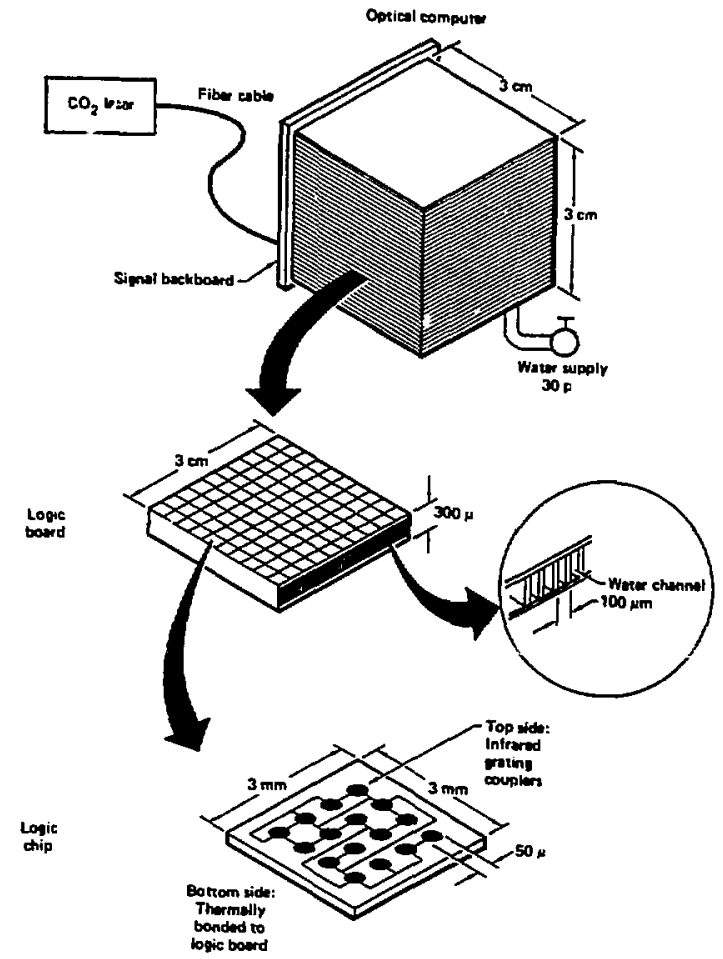

Figure B.5: A possible optical computer physical layout. 
The logic elements will be powered by a $\mathrm{CO}_{2}$ laser outside the computer and beamsplit to couple into the various boards. If the computer consists of 1 million logic elements, each of which consume 50 milliwatts of infrared power, then the $\mathrm{CO}_{2}$ laser must emit 50 kilowatts of infrared light. (This high an infrared power will probably never be needed since improvements will be rade in the logic element before buiding to this scale.) This infrared laser is anticipated to be the most expensive component of the optical computer and cost around $\$ 2,000,000$ if this much power were actually needed. (Future development of the logic element as indicated in the previous section may lead to 10 microwatt logic elements, or an overall computer requirement of only 10 Watts of infrared power, which can be purchased for about $\$ 8,000$.)

The waste heat generated in the logic elements will be dissipated in cooling channels under each board. Each 3 centimeter board will generate about 500 wat.ts of heat. Cooling channeis at 50 psi water pressure with $\mathbf{5 0}$ micron channels and 50 micron fins have been demonstrated to be able to dissipate $790 \mathrm{Watts} / \mathrm{cm}^{2}$ with a thermal resistance of $0.000 \mathrm{~cm}^{2}{ }^{\circ} \mathrm{C} /$ Watt. $[000]$ A similar cooling on the optical logic boards with 50 milliwatt logic elements will have only a $5^{\circ}$ temperature rise. Future low power logic elements will have temperature rises lower by yet another factor of 3000 . Contrary to conventional wisdom, no valid thermal argument against optical logic exists. Rather, it is not clear if terahertz room temperature electronic structrures will ever obtain subfemtojoule switching energies (or even can reach such speeds in a computer system). 